\title{
DETECTING ALGEBRAIC (IN)DEPENDENCE OF EXPLICITLY PRESENTED FUNCTIONS (SOME APPLICATIONS OF NEVANLINNA THEORY TO MATHEMATICAL LOGIC)
}

\author{
R. H. GUREVIČ
}

ABSTRACT. We consider algebraic relations between explicitly presented analytic functions with particular emphasis on Tarski's high school algebra problem.

The part not related directly to Tarski's high school algebra problem. Let $U$ be a connected complex-analytic manifold. Denote by $\mathscr{F}(U)$ the minimal field containing all functions meromorphic on $U$ and closed under exponentiation $f \mapsto e^{f}$. Let $f_{j} \in \mathscr{F}(U), p_{j} \in \mathscr{M}(U)-\{0\}$ for $1 \leq j \leq m$, and $g_{k} \in$ $\mathscr{F}(U), q_{k} \in \mathscr{M}(U)-\{0\}$ for $1 \leq k \leq n$ (where $\mathscr{M}(U)$ is the field of functions meromorphic on $U)$. Let $f_{i}-f_{j} \notin \mathscr{H}(U)$ for $i \neq j$ and $g_{k}-g_{l} \notin \mathscr{H}(U)$ for $k \neq l$ (where $\mathscr{H}(U)$ is the ring of functions holomorphic on $U$ ). If all zeros and singularities of

$$
h=\frac{\sum_{j=1}^{m} p_{j} e^{f_{j}}}{\sum_{k=1}^{n} q_{k} e^{g_{k}}}
$$

are contained in an analytic subset of $U$ then $m=n$ and there exists a permutation $\sigma$ of $\{1, \ldots, m\}$ such that $h=\left(p_{j} / q_{\sigma(j)}\right) \cdot e^{f_{j}-g_{\sigma(j)}}$ for $1 \leq$ $j \leq m$. When $h \in \mathscr{M}(U)$, additionally $f_{j}-g_{\sigma(j)} \in \mathscr{H}(U)$ for all $j$.

On Tarski's high school algebra problem. Consider $L=\{$ terms in variables and $1,+, \cdot, \uparrow\}$, where $\uparrow: a, b \mapsto a^{b}$ for positive $a, b$. Each term $t \in L$ naturally determines a function $\bar{t}:\left(\mathbf{R}_{+}\right)^{n} \rightarrow \mathbf{R}_{+}$, where $n$ is the number of variables involved. For $S \subset L$ put $\bar{S}=\{\bar{t} \mid t \in S\}$.

(i) We describe the algebraic structure of $\bar{\Lambda}$ and $\overline{\mathscr{L}}$, where $\Lambda=\{t \in L \mid$ if $u \uparrow v$ occurs as a subterm of $t$ then either $u$ is a variable or $u$ contains no variables at all $\}$, and $\mathscr{L}=\{t \in L \mid$ if $u \uparrow v$ occurs as a subterm of $t$ then $u \in \Lambda\}$. Of these, $\bar{\Lambda}$ is a free semiring with respect to addition and multiplication but $\overline{\mathscr{L}}$ is free only as a semigroup with respect to addition. A function $\bar{t} \in \bar{S}$ is called +-prime in $\bar{S}$ if $\bar{t} \neq \bar{u}+\bar{v}$ for all $u, v \in S$ and is called multiplicatively prime in $\bar{S}$ if $\bar{t}=\bar{u} \cdot \bar{v} \Rightarrow \bar{u}=1$ or $\bar{v}=1$ for $u, v \in S$. A function is called $(+, \cdot)$-prime in $\bar{S}$ if it is both +-prime and multiplicatively prime in $\bar{S}$. A function in $\bar{\Lambda}$ is said to have content 1 if it is not divisible by constants in $\mathbf{N}-\{1\}$ or by $\neq 1(+, \cdot)$-primes of $\bar{\Lambda}$. The product of functions of content 1 has content 1 . Let $P$ be the multiplicative subsemigroup of $\bar{\Lambda}$ of functions of content 1 . Then $\overline{\mathscr{L}}$ as a semiring is isomorphic to the semigroup semiring $\bar{\Lambda}\left(\oplus_{f} P_{f}\right)$, where each $P_{f}$ is a copy of $P$ and $f$ ranges over the $\neq 1+$-primes of $\overline{\mathscr{L}}$.

Received by the editors February 1, 1987 and, in revised form, April 11, 1989.

1980 Mathematics Subject Classification (1985 Revision). Primary 30B40, 32D15, 03C99, 08B99; Secondary 30D35. 
(ii) We prove that if $t, u \in \mathscr{L}$ and $\mathbf{R}_{+} \vdash t=u$ (i.e., if $\bar{t}=\bar{u}$ ) then \{Tarski's "high school algebra" identities $\} \vdash t=u$. This result covers a conjecture of C. W. Henson and L. A. Rubel. (Note: this result does not generalize to arbitrary $t, u \in L$. Moreover, the equational theory of $\left(\mathbf{R}_{+} ; 1,+, \cdot, \uparrow\right)$ is not finitely axiomatizable.)

\section{NotATION}

$\mathbf{N}=\{1,2,3, \ldots\}$ (the positive integers), $\mathbf{N}_{0}=\mathbf{N} \cup\{0\}, \mathbf{Z}=$ integers $\}$, $\mathbf{Q}=\{$ rational numbers $\}, \mathbf{R}=\{$ reals $\}, \mathbf{R}_{+}=\{$positive reals $\}, \mathbf{C}=\{$ complex numbers $\}, \mathbf{C}_{*}=\mathbf{C}-\{0\}$.

" $\mathscr{L}$ " refers to "holomorphic". In particular, $\mathscr{H}(U)=\{$ functions holomorphic on $U\}, \mathscr{H}_{\zeta}=\{$ holomorphic germs at $\zeta \in U\}, \mathscr{H}^{*}(U)=\{f \in \mathscr{H}(U) \mid \forall u \in$ $U: f(u) \neq 0\}$, and $\mathscr{H}_{\zeta}^{*}=\left\{f \in \mathscr{H}_{\zeta} \mid f(\zeta) \neq 0\right\}$.

" $\mathscr{M}$ " refers to "meromorphic". In particular, $\mathscr{M}_{\zeta}=\{$ the germs meromorphic at $\zeta\}=$ the field of quotients of the ring $\mathscr{H}_{\zeta}$, and $\mathscr{M}(U)=\{$ meromorphic functions on $U\}$. Note that a meromorphic function on $U$ is a section over $U$ of the sheaf $\mathscr{M}$ of the quotient fields of the sheaf of rings $\mathscr{H}$, i.e., is a quotient of holomorphic functions in a neighborhood of every point, and is not necessarily a quotient of holomorphic functions on $U$.

\section{Preface}

Our involvement with this topic began in 1981 when Gregory E. Mints attracted our attention to "Tarski's high school algebra problem". Tarski's quantifier elimination for real-closed fields nowadays is used both in pure mathematics, e.g., in semialgebraic geometry and in ordinary differential equations, (for the latter see [3, §37]), and outside it, e.g., in robotics (see [32] and references there). But Alfred Tarski was interested also in the theory of the reals equipped with a transcendental operation. The most common transcendental operations are the trigonometric ones and exponentiation. The trigonometric operations present an unpleasant perspective for a logician: they permit one to define $\mathbf{Z} \subset \mathbf{R}$ by a first-order formula and consequently they prevent decidability of the corresponding elementary theories and allow one to define subsets of $\mathbf{R}^{n}$ with rather bad topological structure. So Tarski asked a number of logical, model-theoretic, real-analytic, etc. questions on exponentiation. "Exponentiation" sometimes refers to the operation $\exp : z \mapsto e^{z}$ for $z \in \mathbf{C}$, and sometimes to the operation $\uparrow: a, b \mapsto a^{b}$ for $a, b \in \mathbf{R}_{+}$. In the former case it is most natural to consider functions $\mathbf{C}^{m} \rightarrow \mathbf{C}$ represented by terms in constants, variables, and operations $+,-, \cdot, \exp$, and in the latter case it is most natural to consider functions $\mathbf{N}^{m} \rightarrow \mathbf{N}$ or $\left(\mathbf{R}_{+}\right)^{m} \rightarrow \mathbf{R}_{+}$represented by terms in positive (integer) constants, variables, and operations $+, \cdot, \uparrow$. The function classes arising from both exp and $\uparrow$ have received significant attention. The former class is called the (complex) Shanuel class (see, e.g., $[8,19]) .{ }^{1}$ The latter classes, we think, have no established names, but their subset consisting of the functions represented by one-variable terms with positive integer constants is called the Skolem set (see $[7,11,12,15,18,29,34,36])$.

\footnotetext{
${ }^{1}$ There is a noninterrupting (since the Ritt times at least) activity on "exponential polynomials" among analysts and number theorists. Exponential polynomials are linear combinations with constant coefficients of functions of the form $e^{\mu z}$ with $\mu \in \mathbf{C}$.
} 
Another point of view, largely due to V. I. Arnold, is that one should not worry much about decidability but attempt to find analogues of the "projection of a semialgebraic set is semialgebraic" result to larger classes of semianalytic (or similar) sets, and that having achieved this the questions of effectiveness would be easier to approach. And indeed, that was done by $L$. van den Dries, see $[9,10]$. A number of Muscovites attempted to extend various results from géométrie algébrique to géométrie analytique: in particular A. G. Hovanski proved magnificent finiteness results (see his contribution "Fewnomials and Pfaff manifolds" to the International Congress of Mathematicians (Warszawa 1983), and references there).

The topic of this paper is how to detect algebraic and analytic relations between explicitly presented functions, with particular emphasis on Tarski's high school algebra problem.

Denote by $L$ the class of terms in variables and $1,+, \cdot, \uparrow$. Each $t \in L$ naturally determines a function $\bar{t}:\left(\mathbf{R}_{+}\right)^{n} \rightarrow \mathbf{R}_{+}$, where $n$ is the number of variables involved. Suppose $t, u \in L$; we want to know if $\bar{t}=\bar{u}$. This problem is "solvable", i.e., some badly slow algorithms were published (they are primitive recursive, but that leaves open the question of whether a computer can answer in your lifetime " $\bar{t} \stackrel{?}{=} \bar{u}$ " for a couple of 1000-symbol long terms from $L$ ). Further, let $t_{1}, \ldots, t_{n} \in L$. You might want your computer to tell you if $\bar{t}_{1}, \ldots, \bar{t}_{n}$ are algebraically dependent, and if they are, then to provide a nontrivial polynomial $f \in \mathbf{Z}\left[x_{1}, \ldots, x_{n}\right]$ with $f\left(\bar{t}_{1}, \ldots, \bar{t}_{n}\right)=0$. We do not restrict ourselves to $L$, e.g., we are interested in effective ways to detect algebraic and analytic relations between functions presented by expressions like

$$
\left(\tan \frac{x+\cos x}{\Gamma(\Gamma(x))}\right)^{x^{x}}, \quad \sin \left(x^{\sin (1 / x)}\right), \quad\left(e^{x}+\Gamma(\tan x)\right)^{\tan x}
$$

but we prefer to use $L$ as our main testing site. The main reason for this is our far-extending beliefs concerning the algebraic structure of $\bar{L}=\{\bar{t} \mid t \in L\}$ without having any idea on how to prove them.

The main results of the paper. (i) A description of algebraic relations between the functions (on an arbitrary connected complex-analytic manifold) obtained from the meromorphic functions by operations $+,-, \cdot, /, \exp$, where $\exp : f$ $\mapsto e^{f}(\S 2)$.

(ii) A study of the class of functions presented by terms in $\mathscr{L}=\{t \in L \mid$ if $a \uparrow b$ occurs as a subterm of $t$ then $a \in \Lambda\}$, where $\Lambda=\{t \in L \mid$ if $a \uparrow b$ occurs as a subterm of $t$ then $a$ is either a variable or contains no variables at all $\}$. This includes an algebraic description of $\overline{\mathscr{L}}=\{\bar{t} \mid t \in \mathscr{L}\}$ and the proof of Tarski's conjecture on $\mathscr{L}:$ if $t, u \in \mathscr{L}$ and $\bar{t}=\bar{u}$ then the identity $t=u$ can be derived from "high school algebra" identities; see [18]. The latter is stronger than the Henson-Rubel conjecture in [19] that Tarski's conjecture holds on $\Pi=\{t \in L \mid$ if $a \uparrow b$ occurs as a subterm of $t$ then $a$ is a polynomial $\}$.

\section{INTRODUCTION}

\subsection{Introductory examples.}

Example 1. Let $p_{j} \in \mathbf{C}[z]$ and $p_{k}-p_{l} \neq$ const for $k \neq l$. Then the functions $\left\{e^{p_{j}(z)}\right\}_{j}$ are linearly independent over $\mathbf{C}(z)$. 
This is a very special case of the Hiromi-Ozawa lemma (see $§ 1.3$ ) and admits the following elementary proof.

Let $q_{j} \in \mathbf{C}[z]$ and $\sum_{j=1}^{n} q_{j}(z) \cdot e^{p_{j}(z)}=0$. I have to show that $q_{j}=0$ for $j=1, \ldots, n$. Proceed by induction on $n$; the case $n=1$ is trivial. Let $n>1$. For the induction step from $n-1$ to $n$ proceed by induction on $\operatorname{deg}\left(q_{n}\right)$; the case of $\operatorname{deg}\left(q_{n}\right)<0$, i.e., of $q_{n}=0$ is just the induction assumption on $n-1$. Suppose that $q_{n} \neq 0$. We have $\sum_{j=1}^{n-1} q_{j} \cdot e^{p_{j}-p_{n}}+q_{n}=0$, whence by differentiation $\sum_{j=1}^{n-1}\left(q_{j}^{\prime}+q_{j} \cdot\left(p_{j}-p_{n}\right)^{\prime}\right) e^{p_{j}-p_{n}}+q_{n}^{\prime}=0$. Since $\operatorname{deg}\left(q_{n}^{\prime}\right)<\operatorname{deg}\left(q_{n}\right)$ by the induction assumption on $\operatorname{deg}\left(q_{n}\right)$, we have $q_{n}^{\prime}=0$ and $q_{j}^{\prime}=-q_{j} \cdot\left(p_{j}-p_{n}\right)^{\prime}$ for $1 \leq j \leq n-1$. Since $\left(p_{j}-p_{n}\right)^{\prime} \neq 0$ because $p_{j}-p_{k} \neq$ const, we have $\operatorname{deg}\left(q_{j}^{\prime}\right)<\operatorname{deg}\left(q_{j} \cdot\left(p_{j}-p_{n}\right)^{\prime}\right)$ unless $q_{j}=0$. Thus $q_{j}=0$ for $1 \leq j \leq n-1$. Then $q_{n}=0$ too.

Example 2. Let $p_{1}, \ldots, p_{n} \in \mathbf{R}_{+}(x)$ (i.e., rational functions with positive real coefficients) and $q \in \mathbf{C}[x], q \neq$ const. If $p_{j} \neq p_{k}$ for $j \neq k$ then the functions $\left\{\left(p_{j}(z)\right)^{q(z)}\right\}_{j}$ (well-defined on $\mathbf{R}_{+}$) are linearly independent over $\mathrm{C}(z)$.

This is a very special case of other complex-variable results (see $\S \S 3$ and 4) and the proof below introduces one of the basic ideas of the paper.

Let $r_{j} \in \mathbf{C}[z]$ and $\sum_{j=1}^{n} r_{j} \cdot\left(p_{j}\right)^{q}=0$; we have to show that $r_{j}=0$ for $1 \leq j \leq n$. Proceed by induction on $n$. Upon dividing all summands by $\left(p_{1}\right)^{q}$ one can assume that $p_{1}=1$. If $p_{2}, \ldots, p_{n}$ are all regular, i.e., have neither zeros nor poles in $\mathbf{C}$, then they are distinct constants and the statement follows from the result of Example 1.

Suppose that not all of $p_{2}, \ldots, p_{n}$ are regular. Then for at least one $j$ the result of the analytic continuation of $\left(p_{j}\right)^{q}$ into $\mathbf{C}$ is multivalued. Let $z \in \mathbf{C}$ be a zero or a pole of some $\left\{p_{j}\right\}_{j=2}^{n}$. The analytic continuation of $\left(p_{j}\right)^{q}$ along a loop winding counter-clockwise once around $z$ multiplies it by $\exp \left(2 \pi i \mu_{j} q(z)\right)$, where $\mu_{j}$ is the multiplicity of $z$ in $p_{j}, \mu_{j} \in \mathbf{Z}$. Such continuation along a loop winding $k$ times counter-clockwise around $z$ multiplies each $r_{j} \cdot\left(p_{j}\right)^{q}$ by $\exp \left(2 \pi i \mu_{j} q(z) k\right)$. However, the total sum must remain zero: $\sum_{j=1}^{n} r_{j}$. $\left(p_{j}\right)^{q} \cdot \exp \left(2 \pi i \mu_{j} q k\right)=0$. Consider the set $M=\left\{\mu_{j} \mid j=1, \ldots, n\right\} \subset \mathbf{Z}$. Since some $\mu_{j}$ may coincide, $M$ may contain fewer than $n$ elements but, by the assumptions made, $M$ contains more than one element: $0 \in M$ and $M-\{0\} \neq \varnothing$. For each $m \in M$ put $g_{m}=\sum\left\{r_{j} \cdot\left(p_{j}\right)^{q} \mid \mu_{j}=m\right\}$; then $\sum_{m \in M} g_{m} \cdot \exp (2 \pi i m q(z) k)=0$ for all $k \in \mathbf{Z}$. Denote $l=|M|$. One can regard

$$
\left\{\sum_{m \in M} g_{m} \cdot \exp (2 \pi i m q(z) k)=0\right\}_{k=0}^{l-1 .}
$$

as a system of linear equations for $\left\{g_{m}\right\}_{m \in M}$. The determinant of this system has the form

$$
\left|\begin{array}{cccc}
1 & 1 & \cdots & 1 \\
t_{1} & t_{2} & \cdots & t_{l} \\
\vdots & \vdots & & \vdots \\
t_{1}^{l-1} & t_{2}^{l-1} & \cdots & t_{l}^{l-1}
\end{array}\right|
$$


where $t_{k}=\exp \left(2 \pi i m_{k} q(z)\right), M=\left\{m_{1}, \ldots, m_{l}\right\}$. Since $q \neq$ const all $\left\{t_{k}\right\}_{k}$ are distinct, and consequently the (Vandermonde) determinant above is not zero. Hence $g_{m}=0$ for all $m \in M$. Since each $g_{m}$ consists of fewer than $n$ summands, the induction assumption provides the result.

Example 3. Let $\lambda \in \mathbf{C}-\mathbf{Q}$. Then there are no analytic relations between $e^{z}$ and $e^{\lambda z}$, i.e., if $f$ is a function holomorphic in a neighborhood of $(1,1)$ in $\mathbf{C}^{2}$ and $f\left(e^{z}, e^{\lambda z}\right)=0$ whenever this expression is defined then $f=0$ identically.

This statement splits into two totally dissimilar cases: $\lambda \in \mathbf{R}-\mathbf{Q}$ and $\lambda^{*} \epsilon$ $\mathbf{C}-\mathbf{R}$, considered in some detail below. The case of real $\lambda$ can be generalized as follows.

Example 4. Let $\left\{\lambda_{j}\right\}_{j=1}^{n} \subset \mathbf{R}$ be linearly independent over $\mathbf{Q}$, and $f$ be a holomorphic function in $n$ variables whose domain contains at least one point $\left(z_{1}, \ldots, z_{n}\right)$ with $\left|z_{1}\right|^{1 / \lambda_{1}}=\cdots=\left|z_{n}\right|^{1 / \lambda_{n}} \neq 0$. If $f\left(e^{\lambda_{1} z}, \ldots, e^{\lambda_{n} z}\right)=0$ whenever this expression is defined, then $f=0$ identically.

Show first that $\left\{e^{\lambda_{j} z}\right\}_{j=1}^{n}$ belongs to the domain of $f$ for at least one $z$. Let $\left(z_{1}, \ldots, z_{n}\right) \in \operatorname{dom}(f)$ and $r=\left|z_{1}\right|^{1 / \lambda_{1}}=\cdots=\left|z_{n}\right|^{1 / \lambda_{n}} \neq 0$. Consider $z=$ $\log r+i s, s \in \mathbf{R}$. Then $\left|e^{\lambda_{j} z}\right|=\left|z_{j}\right|$ and the argument of $e^{\lambda_{j} z}$ is $\lambda_{j} s(\bmod 2 \pi \mathbf{Z})$. Since the $\lambda_{j}$ 's are linearly independent over $\mathbf{Q}$, by a theorem of Kronecker the set

$$
\left\{\left(e^{i \lambda_{1} s}, \ldots, e^{i \lambda_{n} s}\right) \mid s \in \mathbf{R}\right\}
$$

is dense in the product of $n$ copies of the unit circle. Thus one can approximate $\left(z_{1}, \ldots, z_{n}\right)$ with points of the form $\left(e^{\lambda_{1} z}, \ldots, e^{\lambda_{n} z}\right)$.

Let $z$ be such that $\left\{e^{\lambda_{j} z}\right\}_{j=1}^{n}$ belongs to the domain of $f$. Since $\left\{\lambda_{j}\right\}_{j=1}^{n}$ are linearly independent over $\mathbf{Q}$, by a theorem of Kronecker for any $\varepsilon \in \mathbf{R}_{+}$ and $\left\{\xi_{j}\right\}_{j=1}^{n} \subset\left(S^{1}\right)^{n}$ (where $S^{1}$ is the unit circle in $\mathbf{C}$ ) there exists a real $\theta$ such that $\max _{j}\left|\xi_{j}-e^{i \lambda_{j} \theta}\right|<\varepsilon$. Since $f\left(\left\{e^{\lambda_{j} z} \cdot e^{i \lambda_{j} \theta}\right\}_{j=1}^{n}\right)=0$ whenever this expression is defined, by continuity $f\left(\left\{e^{\lambda_{j} z} \cdot \xi_{j}\right\}_{j=1}^{n}\right)=0$ whenever all $\xi_{j}$ have modulus 1 and are sufficiently close to 1 . Since $f$ is holomorphic this implies that $f\left(\left\{e^{\lambda_{j} z} \cdot \xi_{j}\right\}_{j=1}^{n}\right)=0$ whenever all $\xi_{j}$ are sufficiently close to 1 , whence $f\left(\left\{w_{j}\right\}_{j=1}^{n}\right)=0$ whenever this latter expression is defined.

The second case of Example 3, of nonreal $\lambda$, cannot be generalized the way the case of $\lambda$ real was.

Example 5. Let $\lambda \in \mathbf{C}-\mathbf{R}$. The mapping $h: z \mapsto\left(e^{z}, e^{\lambda z}\right)$ is a proper embedding of $\mathbf{C}$ into $(\mathbf{C}-\{0\})^{2}$. Since $(\mathbf{C}-\{0\})^{2}$ is a Stein manifold, $h(\mathbf{C})$ being a complex-analytic submanifold is also a variety ${ }^{2}$ in $(\mathbf{C}-\{0\})^{2}$. Let $\lambda, \mu \in \mathbf{C}$ with $\operatorname{re} \lambda, \operatorname{re} \mu<0$ and $\operatorname{im} \lambda<0<\operatorname{im} \mu$. Then $g: z \mapsto\left(e^{z}, e^{\lambda z}, e^{\mu z}\right)$ is a proper embedding of $\mathbf{C}$ in $\mathbf{C}^{3}$. Thus again $g(\mathbf{C})$ is a variety.

The statement that $g(\mathbf{C})$ is a variety for $g: z \mapsto\left(e^{z}, e^{\lambda z}, e^{\mu z}\right)$, re $\lambda$, re $\mu<$ $0, \operatorname{im} \lambda<0<\operatorname{im} \mu$, tells us only that there exists an entire function in three variables which is not identically zero but $h\left(e^{z}, e^{\lambda z}, e^{\mu z}\right)=0$. How complicated is it? If $1, \lambda, \mu$ are linearly independent over $\mathbf{Q}$, then obviously $h$ cannot be a polynomial. Moreover, then (we can prove that) $h(u, v, w)$ cannot be obtained from the variables $u, v, w$ and constants by means of operations

\footnotetext{
2"Variety in $U$ " means "the zero set of an ideal in $\mathscr{H}(U)$ ".
} 
$+,-, \cdot, \exp$ (in other words, $h$ cannot belong to the Schanuel class). It is easy to see that the mapping $z \mapsto\left(e^{\lambda_{1} z}, \ldots, e^{\lambda_{n} z}\right)$ is a proper embedding of $\mathbf{C}$ into $\mathbf{C}^{n}$ if and only if 0 belongs to the interior of the convex hull of $\left\{\lambda_{1}, \ldots, \lambda_{n}\right\}$. This fact provides one more necessary condition for analytic independence of $e^{\lambda_{1} z}, \ldots, e^{\lambda_{n} z}$ (in addition to the linear independence of $\lambda_{1}, \ldots, \lambda_{n}$ over $\mathbf{Q}$ ). We do not know whether these two necessary conditions are sufficient. The second case of Example 3 is contained in the following.

Example 6. Let $\lambda_{1}, \ldots, \lambda_{n} \in \mathbf{R}$ be linearly independent over $\mathbf{Q}$ and $\mu \epsilon$ $\mathbf{C}-\mathbf{R}$. If $f$ is holomorphic in a neighborhood of a point $w=\left(w_{1}, \ldots, w_{n}, 0\right)$ in $\mathbf{C}^{n+1}$ with $\left|w_{1}\right|^{1 / \lambda_{1}}=\cdots=\left|w_{n}\right|^{1 / \lambda_{n}} \neq 0$ and $f\left(e^{\lambda_{1} z}, \ldots, e^{\lambda_{n} z}, e^{\mu z}\right)=0$ whenever this expression is defined, then $f=0$ identically.

Suppose the contrary: let $f$ not be identically zero. Without loss of generality one can assume that the germ of $f$ at $w \in \mathbf{C}^{n+1}$ is irreducible. Besides, one can assume that $\operatorname{im} \mu>0$. Let $U$ be a polydisk centered at $w$ and contained in the domain of $f$. As in Example 4 one can easily see that there exists a $z \in \mathbf{C}$ such that $\left(\left\{e^{\lambda_{j} z}\right\}_{j=1}^{n}, e^{\mu z}\right) \in U$. By Kronecker's theorem, for any $\varepsilon, M \in \mathbf{R}_{+}$and any $\left\{\xi_{j}\right\}_{j=1}^{n} \subset\left(S^{1}\right)^{n}$ (where $S^{1}$ is the unit circle in C) there exists a real $\theta>M$ such that $\max _{j}\left|\xi_{j}-e^{i \lambda_{j} \theta}\right|<\varepsilon$. Note that $\left|e^{\mu(z+i \theta)}\right|<\left|e^{\mu z}\right| \cdot \exp (-\operatorname{im} \mu \cdot M)$. Hence by continuity $f\left(\left\{e^{\lambda_{j} z} \cdot \xi_{j}\right\}_{j=1}^{n}, 0\right)=0$ whenever all $\xi_{j}$ have modulus 1 and are sufficiently close to 1 . Since $f$ is holomorphic, then $f\left(\left\{e^{\lambda_{j} z} \cdot \xi_{j}\right\}_{j=1}^{n}, 0\right)=0$ whenever all $\xi_{j}$ are sufficiently close to 1 , whence $f\left(\left\{w_{j}\right\}_{j=1}^{n}, 0\right)=0$ whenever this latter expression is defined. Then $f$ is divisible by $w_{n+1}$; and since $f$ is irreducible, $f=w_{n+1}$. (a unit). But $w_{n+1}$ does not vanish on $z \mapsto\left(\ldots, e^{\mu z}\right)$.

The examples below come nearer to what we do in this paper. Their proofs are also examples-of how to use the technique developed in the paper.

Example 7. Let $f, g$ be meromorphic in a neighborhood of 0 in $\mathbf{C}, f$ have a zero or a pole at 0 , and $g$ not be a rational constant. Let $f(\zeta) \in \mathbf{C}-\{0\}$, $g(\zeta) \in \mathbf{C}$; fix a value for $\log f(\zeta)$. Then $f^{g}$ (a holomorphic germ at $\zeta$ ) is analytically transcendental, i.e., if $h(w, u)$ is holomorphic in a neighborhood of $\{\zeta\} \times \mathbf{C}$ in $\mathbf{C}^{2}$ and $h\left(w, f(w)^{g(w)}\right)=0$ in a neighborhood of $\zeta$ then $h$ is identically zero.

Consider first the case of $g \in \mathbf{R}-\mathbf{Q}$. The analytic continuation along a loop winding around 0 multiplies $f^{g}$ by $\exp (2 \pi i g \mathrm{~km})$, where $k$ is the multiplicity of $f$ at $0 \quad(k \neq 0)$ and $m$ is the winding number. However, $h\left(w, e^{2 \pi i g k m} \cdot f^{g}\right)$ has to remain 0 . By Kronecker's theorem the set $\left\{e^{2 \pi i g k m} \mid m \in \mathbf{Z}\right\}$ is dense in the unit circle, and hence $h\left(w, \xi \cdot f^{g}\right)=0$ whenever $|\xi|=1$. Since $h$ is holomorphic, $h\left(w, \xi \cdot f^{g}\right)=0$ for all $\xi \in \mathbf{C}$, and thus $h=0$ identically.

Let $g \notin \mathbf{R}$. Without loss of generality one can assume that $h$ is not divisible by $u$, i.e., the zero set of $h$ does not contain $\mathbf{C} \times\{0\}$. The analytic continuation along a loop winding around 0 multiplies $f^{g}$ by $\exp (2 \pi i g(w) \mathrm{km})$, where $k$ is the multiplicity of $f$ at 0 and $m$ is the winding number. Choosing $w$ with $g(w) \notin \mathbf{R}$ and a sequence $\left\{m_{\nu}\right\}_{\nu}$ one can make $\exp \left(2 \pi i g(w) k m_{\nu}\right)$ converge to zero. Thus $h(w, 0)=0$ for $w$ with $g(w) \notin \mathbf{R}$, and consequently $h(w, 0)=0$ for all $w$, the latter contradicting the assumption on $h$. 
Example 8. Let $f, g$ be holomorphic in a neighborhood of $[0, \pi / 2]$ and not be rational constants. Then $(\sin x)^{f(x)}$ and $(\cos x)^{g(x)}$ are analytically independent, viz. if $h(z, u, v)$ is holomorphic in a neighborhood of $[0, \pi / 2] \times \mathbf{C}^{2}$ and $h\left(x,(\sin x)^{f},(\cos x)^{g}\right)=0$ on $(0, \pi / 2)$ then $h$ is identically 0 .

If $f$ and $g$ are real constants then by the same reasoning as in the previous example $h\left(x, \xi \cdot(\sin x)^{f}, \eta \cdot(\cos x)^{g}\right)=0$, where $\xi$ and $\eta$ are new independent complex variables (continue analytically $(\sin x)^{f}$ around 0 and $(\cos x)^{g}$ around $\pi / 2$ ), and hence $h=0$ identically.

Let at least one of $f, g$ not be in $\mathbf{R}$. Without loss of generality one can assume that $h(z, u, v)$ is not divisible by $u$ or $v$, i.e., that the zero set of $h$ contains neither $[0, \pi / 2] \times\{0\} \times \mathbf{C}$ nor $[0, \pi / 2] \times \mathbf{C} \times\{0\}$. Let $w$ be sufficiently close to $[0, \pi / 2]$ and let at least one of $\operatorname{im} f(w)$, im $g(w)$ be nonzero. The analytic continuation along a loop winding around 0 or $\pi / 2$, respectively, leaves one of $(\cos w)^{g}$ and $(\sin w)^{f}$ intact while the other one is multiplied by $\exp (2 \pi i f(w) m)$ or $\exp (2 \pi i g(w) m)$, where $m$ is the winding number. By choosing a sequence $\left\{m_{\nu}\right\}_{\nu}$ one can make the appropriate one of $\exp \left(2 \pi i f(w) m_{\nu}\right)$ and $\exp \left(2 \pi i g(w) m_{\nu}\right)$ converge to zero. Thus

$$
h\left(w,(\sin w)^{f(w)}, 0\right)=0 \text { or } h\left(w, 0,(\cos w)^{g(w)}\right)=0 .
$$

By the arbitrariness in the choice of $w$, at least one of the above " $=0$ " holds identically. By the previous example, $h(w, 0, v)=0$ or $h(w, u, 0)=0$ identically, contradicting the assumption on $h$.

Example 9. Let $f, g$ be nonconstant entire functions such that both $f(\zeta), g(\zeta)$ $\in \mathbf{R}_{+}$for some $\zeta$ (to have the exponentiations below defined in the usual way at $\zeta)$. If there exists an entire $h(z, u, v)$, not identically zero, such that

$$
h\left(z,\left(f(z)^{\sqrt{2}}+1\right)^{\sqrt{2}},\left(g(z)^{\sqrt{2}}+1\right)^{\sqrt{2}}\right)=0,
$$

then $f=g$.

Of course, this is not a statement on mysterious properties of $\sqrt{2}$ or +1 . One possible generalization is

Example 10. Let $f, g$ be nonconstant entire functions, $P$ be a polynomial in $w$ with coefficients in $\mathbf{C}(z), \operatorname{deg}_{w}(P)>0$, and $P$ not be divisible by $w$. Let $\lambda, \mu \in \mathbf{R}-\mathbf{Q}$. Take a point $\zeta \in \mathbf{C}^{n}$ and the values of logarithms needed such that $a=\left(P\left(z, f^{\lambda}\right)\right)^{\mu}$ and $b=\left(P\left(z, g^{\lambda}\right)\right)^{\mu}$ are both well defined in a neighborhood of $\zeta$. If there exists an entire $h(w, u, v)$, not identically zero, such that $h(w, a(w), b(w))=0$ in a neighborhood of $\zeta$, then $f=g$.

The proof is analogous to, but is more difficult, than the proofs of the previous examples. Again, the underlying idea is to continue the germs analytically and to describe the monodromy, i.e., how the results of the analytic continuation are related to the original germs.

1.2. On Tarski's high school algebra problem. In this section we discuss briefly the aspects of Tarski's high school algebra problem dealt with in this paper. We state several conjectures, present examples, and make some remarks. Then we present some basic results and prove the "it is easy to see" statements of this section. Reminder: $\mathbf{N}$ does not contain $0, \mathbf{N}_{0}=\mathbf{N} \cup\{0\}$. 
Let $\uparrow$ be the binary operation symbol for $a, b \mapsto a^{b}$. For a set $V$ of variables let $L(V)$ be the set of terms built up from variables in $V$ and constants for natural numbers with operations $+, \cdot, \uparrow$. Each $a \in L(V)$ determines naturally a positive real function $\bar{a}$ on the positive orthant $\left(\mathbf{R}_{+}\right)^{V}$ of $\mathbf{R}^{V}$.

The following question of A. Tarski was popularized by L. Henkin in [18]: if two terms from $L(V)$ represent the same function on $\left(\mathbf{R}_{+}\right)^{V}$ can their identity then be derived from the high school algebra axioms? The "high school algebra axioms" are

(1) $1^{x}=1, x^{1}=1 x=x 1=x$

(2) $x+y=y+x, x y=y x$

(3) $x+(y+z)=(x+y)+z, x(y z)=(x y) z$,

(4) $x(y+z)=x y+x z$

(5) $x^{y+z}=x^{y} x^{z}$

(6) $x^{z} y^{z}=(x y)^{z}$

(7) $x^{y z}=\left(x^{y}\right)^{z}$.

It is convenient to have the constants for all natural numbers and the defining axioms for the constants: const ${ }_{n+1}=$ const $_{n}+1$. Then $\mathscr{T}=\{$ the axioms (1)-(7) $\}$ $\cup\{$ the defining axioms for the constants $\}$. Terms $t, u \in L$ will be called $\mathscr{T}$ equivalent, written $t=\mathscr{T} u$, if $\mathscr{T} \vdash t=u$. The above question then is: for $t, u \in L$ does $\mathbf{R}_{+} \vDash t=u$ imply $t=g u$ ? This question was answered negatively by A. J. Wilkie in [36]; see [18] for earlier results and [15] for some later results. The assertion that for terms from a particular subclass of $L(V)$ the functional identity implies the derivability is often referred to as Tarski's conjecture for that class.

Let $\bar{L}(V)=\{\bar{a} \mid a \in L(V)\}$. What can be said about arithmetic properties of $\bar{L}(V)$ ? One of the reasons to ask such a question is the fact that in cases where Tarski's conjecture was proved it was actually derived from the arithmetic properties of the classes considered. It is also interesting to compare the arithmetic properties of $\bar{L}$ and of the class of polynomials with positive integer coefficients. The classes of positive integer and real polynomials were considered in connection with ergodic theory and some other topics; see, e.g., [16].

The functions in $\bar{L}$ are real-analytic. If in $\bar{t}: \mathbf{N}^{m} \rightarrow \mathbf{N}, t \in L$, all indeterminates except one are fixed then the resulting function is in $\bar{L}$ (the remaining indeterminate). The set $\mathscr{S} k=\bar{L}(\{x\})$ of functions $\mathbf{R}_{+} \rightarrow \mathbf{R}_{+}$is called the Skolem set. $\mathscr{S} k$ is contained in every maximal Hardy field ${ }^{3}$ (see [5] and [6]) and all nonconstant functions in $\mathscr{S} k$ are eventually (i.e., for sufficiently large values of the variable) increasing. $\mathscr{S}_{k}$ is known to be well ordered by the relation $f \prec g \Leftrightarrow \exists x: \forall y>x: f(y)<g(y)$; see [12]. The order type of $(\mathscr{S} k, \prec)$ is $\geq \varepsilon_{0}$ and is $\leq$ the first fixed point of the $\varepsilon$-function; see [29]. ${ }^{4}$ It is a common conjecture that $\varepsilon_{0}$ is actually the order type of $(\mathscr{S} k, \prec)$.

Definition. Let $\$$ stand for any of $+, \cdot, \uparrow$. A function $f \in \bar{L}(V)$ will be called $\$$-prime if for any $g, h \in \bar{L}(V)$ with $f=g \$ h$ we have $f=g$ or $f=h$.

\footnotetext{
${ }^{3}$ A Hardy field is a differential field $\mathscr{B}$ of $C^{\infty}$ function germs at $+\infty$ in $\mathbf{R}_{+}$. If $f \in \mathscr{B}$ then $f(x) \neq 0$ for all sufficiently large $x$ (to have $1 / f \in \mathscr{B}$ ), and since $g^{\prime} \in \mathscr{B}$ for any $g \in \mathscr{B}$, each $f(x) \in \mathscr{B}$ is monotonic for all sufficiently large $x$.

${ }^{4}$ Reminder: $\varepsilon_{0}$ is the first ordinal number $\varepsilon$ satisfying $\varepsilon=\omega^{\varepsilon}$; see [25].
} 
Since $0 \notin \bar{L}(V)$ this definition implies that a +-prime function in $\bar{L}(V)$ is one which is not a sum of functions from $\bar{L}(V)$.

Examples. The constant 1 is additively, multiplicatively, and exponentially prime or, shorter, $(+, \cdot, \uparrow$ )-prime. Let $v \in V$. Then (for the functions generated by the terms shown) it is easy to see that:

(i) $v$ is $(+, \cdot, \uparrow)$-prime,

(ii) $v+1$ is $(\cdot, \uparrow)$-prime but not +-prime,

(iii) $v \cdot 2^{v}$ is $(+, \uparrow)$-prime but not multiplicatively prime,

(iv) $2^{v}$ is $(+, \cdot)$-prime but not $\uparrow$-prime,

(v) $v^{2}+3 v+2$ is $\uparrow$-prime only,

(vi) $v^{2}$ is +-prime only,

(vii) $v^{2}+2 v+1$ is not prime with respect to all of $+, \cdot, \uparrow$.

The absence from this list of a function multiplicatively prime only is not an accident. It is easy to see (Corollary 3 below) that each function in $\bar{L}(V)$ is a sum of +-primes, and that each +-prime can be presented in the form

$$
\text { (a monic monomial } \in \mathbf{Z}[V]) \cdot \prod_{j}\left(g_{j}\right)^{h_{j}},
$$

where each $\left(g_{j}\right)^{h_{j}}$ is $(+, \cdot)$-prime, $\neq 1$ (then $g_{j}$ is multiplicatively prime and $h_{j}$ is a +-prime), and $h_{j} \neq 1$ for each $j$.

Conjecture 1. If $u$ is a monic monomial in $\mathbf{Z}[V],\left\{g_{j}\right\}_{j} \subset \bar{L}(V),\left\{h_{j}\right\}_{j} \subset$ $\bar{L}(V)-\{1\}$, and each $h_{j}$ is +-prime then $u \cdot \prod_{j}\left(g_{j}\right)^{h_{j}}$ is +-prime.

An immediate consequence of Conjecture 1 would be: if $h$ is multiplicatively prime then $h$ is +-prime or $\uparrow$-prime. Indeed, let $h=p^{q}$ and $p \neq 1$; $q \neq 1$. Then $q$ must be +-prime (otherwise $h$ would not be multiplicatively prime) and by the conjecture $h$ will be +-prime.

Conjecture 2. If $h_{1}, \ldots, h_{m} \in \bar{L}(V)$ are +-prime and $h_{i} \neq h_{j}$ for $i \neq j$ then $\left\{h_{1}, \ldots, h_{m}\right\}$ is linearly independent over $\mathbf{R}$.

An immediate consequence of Conjecture 2 would be: every function in $\bar{L}(V)$ admits only one decomposition into +-prime summands.

Whereas uniqueness of decomposition into sums of +-primes is plausible, even a polynomial can have more than one decomposition into products of multiplicatively prime factors in $\bar{L}(V)$ :

$$
(x+1)\left(x^{4}+x^{2}+1\right)=\left(x^{3}+1\right)\left(x^{2}+x+1\right) .
$$

This example is crucial in Wilkie's counterexample to Tarski's conjecture (see [15] for some related results).

Conjectures 1 and 2 can be relativized to any subclass $S$ of $L$ by replacing $\bar{L}(V)$ with $\bar{S}(V)=\{\bar{a} \mid a \in S(V)\}$ in their formulations and in the definition of $\$$-primality $(\$=+, \cdot, \uparrow)$. Note that if Conjectures 1 and 2 hold for $\bar{L}(\{x\})$ (only one variable) then they hold for any $\bar{L}(V)$ (a proof is provided below in this section). L. van den Dries and H. Levitz in [11] (see also [7]) proved Conjecture 1 for the functions $\mathbf{R}_{+} \rightarrow \mathbf{R}_{+}$represented by terms in $\{t \in L(\{x\}) \mid$ if $a^{b}$ occurs as a subterm of $t$ then $b$ is either $x$ or a constant $\}$. For some very specific terms Conjectures 1 and 2 were proved by ad hoc methods in [15]. 
In [19] C. W. Henson and L. A. Rubel considered two classes of terms of $L(V)$ in which exponentiation is permitted only for certain bases: $\Sigma(V)=\{a \in L(V) \mid$ if $b^{c}$ occurs as a subterm of $a$ then $b$ is a constant term $\}$ (the Schanuel class), and $\Lambda(V)=\left\{a \in L(V) \mid\right.$ if $b^{c}$ occurs as a subterm of $a$ then $b \in V$ or $b$ is a constant term $\}$ (the Levitz class). They proved in particular (Conjectures 1 and 2 and as a consequence) Tarski's conjecture for $\Lambda$ and asked about that for the class $\Pi(V)=\left\{a \in L(V) \mid\right.$ if $b^{c}$ occurs as a subterm of $a$ then $b$ is a polynomial $\}$. In $\S 4.4$ of this paper we prove that Conjectures 1,2 and Tarski's conjecture hold not only for $\Pi$ but even for a larger (similar) class. We give an arithmetic conjecture which explains in much more detail my thoughts on $L$ and $\bar{L}$ but before it can be stated some definitions are needed.

Definition. For a polynomial $P \in \mathbb{Z}\left[x_{1}, \ldots, x_{n}\right]-\{0\}$, the content of $P$ is the monomial $m \cdot x_{1}^{l_{1}} \cdots x_{n}^{l_{n}}$, where $m \in \mathbf{N}$ is the greatest common divisor of the coefficients of $P$ and each $l_{i}$ is the minimal degree with which the indeterminate $x_{i}$ occurs in $P$.

This definition of content slightly differs from the usual one; see [26]. Clearly every polynomial in $\mathbf{N}_{0}\left[\left\{x_{i}\right\}_{i}\right]-\{0\}$ can be uniquely presented as the product of its content and a polynomial in $\mathbf{N}_{0}\left[\left\{x_{i}\right\}_{i}\right]-\{0\}$ with content $=1$.

Definition. Let $\Omega$ be a subset of $\bar{L}(V)$ algebraically independent over $\mathbf{Q}$. For any function $f$ generated from functions in $\Omega$ by addition and multiplication (i.e., for $f \in \mathbf{N}_{0}[\Omega]-\{0\}$ ) put the content of $f=$ the function corresponding to the content of $f$ considered as a polynomial in the set of indeterminates $\Omega$.

For any subclass $\Xi$ of $L$ denote $\mathscr{G}(\Xi)=\left\{a \in L \mid\right.$ if $b^{c}$ occurs as a subterm of $a$ then $\left.b \in \mathbf{N}_{0}[\Xi]\right\}$.

Conjecture 3. Let $\Xi$ be a subclass of $L$ such that the set $\bar{\Xi}$ of functions is algebraically independent over $\mathbf{Q}$, and such that if $a \$ b \in \Xi$ for any $a, b \in L$, $\$ \in\{+, \cdot, \uparrow\}$, then $a, b \in \mathbf{N}_{0}[\Xi]$. Then

(i) every function in $\overline{\mathscr{G}(\Xi)}$ can be uniquely decomposed into a sum of +-primes,

(ii) every +-prime in $\overline{\mathscr{G}(\Xi)}$ can be uniquely presented in the form

(a monic monomial $\in \mathbf{Z}[V]$ )

$$
\begin{aligned}
& \times \prod_{i}(\text { a prime } \in \mathbf{N})_{i} \uparrow(\text { a }+ \text {-prime } \in \overline{\mathscr{G}(\Xi)}, \neq 1)_{i} \\
& \times \prod_{j}(\text { a variable })_{j} \uparrow(\text { a }+ \text {-prime } \in \overline{\mathscr{G}(\Xi)}, \neq 1)_{j} \\
& \times \prod_{k}\left(\text { a function } \in \mathbf{N}_{0}[\bar{\Xi}], \neq 1 \text {, with content }=1\right)_{k} \\
& \uparrow(\text { a +-prime } \in \overline{\mathscr{G}(\Xi)}, \neq 1)_{k},
\end{aligned}
$$

where some (or all) of $\prod_{i}, \Pi_{j}, \Pi_{k}$ may be empty and in each of them all occurring exponents are distinct, and the content is taken with respect to $\bar{\Xi}$,

(iii) the functions in $\overline{\mathscr{G}(\Xi)}$ which can be presented in the form $\left(\nabla_{\Xi}\right)$ are +-prime. 
Assuming Conjecture 3 for $\Xi=$ the variables $\}$ (then $\mathscr{G}(\Xi)=\Pi$ ) one can derive from it the following:

the +-primes of $\bar{\Lambda}$ are +-prime in $\Pi$, the multiplicative primes of $\bar{\Lambda}$ are multiplicatively prime in $\Pi$, the $(+, \cdot)$-primes of $\bar{\Lambda}$ (sans 1 ) are algebraically independent.

The third of these statements was actually proved in [19] but was presented in another form. We provide a simplified proof of it in $\S 1.4$. The first two of the above statements will be considered in $\S 3.3$ (which contains some base-ofinduction lemmas) and then in $\S 4.4$ they will be proved together with several related results. In particular, in $\S 4.4$ of this paper we prove that Conjecture 3 is valid for $\bar{\Xi}=\{$ the $(+, \cdot)$-primes of $\bar{\Lambda}\}-\{1\}$.

Proposition 1. Let $p \in L$ and let $v$ be a variable symbol occurring in $p$. If $\mathbf{N} F$ ( $p$ does not depend on $v$ ) then one can erase a number of symbols from $p$ in such a way that the remaining string of symbols will be an L-term $q$ not containing $v$ and $\mathscr{T}$-equivalent to $p$.

In particular, if $\mathbf{N} \vDash p=1$ then that $q$ will be 1 , if $\mathbf{N} \vDash p=($ some variable $y)$ then that $q$ will be $y$.

Let $V$ be a set of variables, $t \in L(V)$, and $\$ \in\{+, \cdot, \uparrow\}$. If $\bar{t}$ is \$-prime in $\bar{L}(V)$ then it is \$-prime in every $\bar{L}(U), U \supset V$. In particular, if $f \in \mathscr{S}_{k}$ is \$-prime in $\mathscr{S} k$ then it is \$-prime in any $\bar{L}(V)$.

Proof. Proceed by induction on the construction of $p$. Note that $p$ can be neither a constant symbol (since $p$ contains $v$ ) nor a variable symbol (because then $p$ would be just $v$, whereas $\bar{p}$ must not depend on $v$ ). The statements which follow provide the induction steps. They all are immediate consequences of the fact that the nonconstant elements of $\mathscr{S} k$ are eventually monotonically increasing. If $\mathbf{N} \vDash(r+s$ does not depend on $v$ ) then $\mathbf{N} \vDash$ (both $r, s$ do not depend on $v$ ), and the same statements apply about $r s$ in place of $r+s$. If $\mathbf{N} \vDash\left(r^{s}\right.$ does not depend on $v$ ) then $\mathbf{N} \vDash r=1$ (in this case one can erase the whole of $s$ ) or $\mathbf{N} \vDash$ (both $r, s$ do not depend on $v$ ).

For the second statement of the proposition note that always $\mathbf{N} \vDash r+s \neq 1$, $\mathbf{N} \vDash r+s \neq y$, and that $\mathbf{N} \vDash r s=1$ implies $\mathbf{N} \vDash r=s=1$ (then one can erase any $r, s), \mathbf{N} \vDash r^{s}=1$ implies $\mathbf{N} \vDash r=1$ (then one can erase $s$ ), $\mathbf{N} \vDash r s=y$ implies $\mathbf{N} \vDash(r=1 \& s=y$ or $r=y \& s=1), \mathbf{N} \vDash r^{s}=y$ implies $\mathbf{N} \vDash(r=y \& s=1)$.

With each $f \in \bar{L}(V)$, one can associate $f(\overrightarrow{2})=f(2,2, \ldots, 2) \in \mathbf{N}$. This provides a $(+, \cdot, \uparrow)$-homomorphism $\bar{L} \rightarrow \mathbf{N}$ with very nice properties. Some examples are given in the following propositions.

Proposition 2. Let $f \in \bar{L}$.

(i) If $f(\overrightarrow{2})=1$ then $f=1$.

(ii) If $f(\overrightarrow{2})=2$ then either $f=2$ or $f=\bar{y}$ for some variable $y$.

(iii) If $f(\overrightarrow{2})=3$ then either $f=3$ or $f=1+\bar{y}$ for some variable $y$.

Of these examples (i) will be used in this paper to prove various statements about $\bar{L}$ by induction on $f(\overrightarrow{2}) \in \mathbf{N}$.

Proof. (i) Consider $p \in L$ satisfying $\bar{p}(\overrightarrow{2})=1$ and proceed by induction on the construction of $p$. The cases of $p$ being a constant or a variable are trivial. 
Further, such $p$ cannot be a sum. Consider $p=q \cdot r$. Then $\bar{q}(\overrightarrow{2}) \cdot \bar{r}(\overrightarrow{2})=$ 1 , whence $\bar{q}(\overrightarrow{2})=\bar{r}(\overrightarrow{2})=1$, and by the induction assumption $\bar{q}=\bar{r}=1$. Consider $p=q \uparrow r$. Then $\bar{q}(\overrightarrow{2})^{\vec{r}(\overrightarrow{2})}=1$, whence $\bar{q}(\overrightarrow{2})=1$, and by the induction assumption $\bar{q}=1$. Hence $\bar{p}=1$.

(ii) Consider $p \in L$ satisfying $\bar{p}(\overrightarrow{2})=2$ and proceed by induction on the construction of $p$. The cases of $p$ being a constant or a variable are trivial. Consider $p=q+r$. Then $\bar{q}(\overrightarrow{2})+\bar{r}(\overrightarrow{2})=2$, whence $\bar{q}(\overrightarrow{2})=\bar{r}(\overrightarrow{2})=1$, and by (i) $\bar{q}=\bar{r}=1, \bar{p}=2$. Consider $p=q \cdot r$. Then $\bar{q}(\overrightarrow{2}) \cdot \bar{r}(\overrightarrow{2})=2$, whence one of $\bar{q}(\overrightarrow{2}), \bar{r}(\overrightarrow{2})$ is 1 and the other is 2 . Consequently one of $\bar{q}, \bar{r}$ is 1 by (i), and the other is 2 or $\bar{y}$ for a variable $y$ by the induction assumption. Consider $p=q \uparrow r$. Then $\bar{q}(\overrightarrow{2})^{\vec{r}(\overrightarrow{2})}=2$, whence $\bar{q}(\overrightarrow{2})=2$ and $\bar{r}(\overrightarrow{2})=1$. Consequently $\bar{r}=1$ by (i), and $\bar{q}$ is either 2 or $\bar{y}$ for a variable $y$ by the induction assumption.

(iii) Consider $p \in L$ satisfying $\bar{p}(\overrightarrow{2})=3$ and proceed by induction on the construction of $p$. The cases of $p$ being a constant or a variable are trivial. Consider $p=q+r$. Then $\bar{q}(\overrightarrow{2})+\bar{r}(\overrightarrow{2})=3$, whence one of $\bar{q}(\overrightarrow{2}), \bar{r}(\overrightarrow{2})$ is 1 and the other is 2 . Then one of $\bar{q}, \bar{r}$ is 1 by (i), and the other is either 2 or $\bar{y}$ for a variable $y$ by (ii). Consider $p=q \cdot r$. Then $\bar{q}(\overrightarrow{2}) \cdot \bar{r}(\overrightarrow{2})=3$, whence one of $\bar{q}(\overrightarrow{2}), \bar{r}(\overrightarrow{2})$ is 1 and the other is 3 . Consequently one of $\bar{q}, \bar{r}$ is 1 by (i), and the other is either 3 or $1+\bar{y}$ for a variable $y$ by the induction assumption. Consider $p=q \uparrow r$. Then $\bar{q}(\overrightarrow{2})^{r}(\overrightarrow{2})=3$, whence $\bar{q}(\overrightarrow{2})=3$ and $\bar{r}(\overrightarrow{2})=1$. Consequently $\bar{r}=1$ by (i), and $\bar{q}$ is either 3 or $1+\bar{y}$ for a variable $y$ by the induction assumption.

Corollary 3. Let $S \subset L$. Then every $f \in \bar{S}$ is a sum of +-primes of $\bar{S}$ and a product of multiplicative primes of $\bar{S}$. If $a \uparrow b \in S \Rightarrow a, b \in S$ for any $a, b \in L$ and $S$ is closed under the multiplication in $L$ then each $f \in \bar{S}$ can be presented as $p^{q}$ with $p, q \in \bar{S}$ and $p$ exponentially prime.

Proof. Proceed by induction on $n=f(\overrightarrow{2})$. If $n=1$ then by the proposition above $f$ is $(+, \cdot, \uparrow)$-prime. Let $n>1$. If $f$ is not +-prime, $f=g_{1}+g_{2}$, then $g_{1}(\overrightarrow{2})<n$ and $g_{2}(\overrightarrow{2})<n$, and by the induction assumption $g_{1}$ and $g_{2}$ are sums of +-primes. If $f$ is not multiplicatively prime, $f=g_{1} g_{2}, g_{1}, g_{2} \neq 1$, then $1<g_{1}(\overrightarrow{2})<n$ and $1<g_{2}(\overrightarrow{2})<n$, and by the induction assumption $g_{1}$ and $g_{2}$ are products of multiplicative primes. If $f$ is not $\uparrow$-prime, $f=$ $p^{q}, p, q \neq 1$, then $1<p(\overrightarrow{2})<n$ and by the induction assumption $p=r^{s}$ with $r$ exponentially prime. Then $f=r^{s q}$.

To prove that the restriction of Conjectures 1 and 2 to $\mathscr{S} k$ imply those on any $\bar{L}(V)$ we proceed by induction on the cardinality of $V$ as follows.

Proposition 4. Let $V \neq \varnothing$ be a set of variables and $y \notin V$ be one more variable. If Conjectures 1 and 2 hold in $\bar{L}(V)$ then

(i) for $x \in V$ and $k \in \mathbf{N}$, if $f \in \bar{L}(V \cup\{y\})$ is +-prime, then the result of substituting $x^{k}$ for $y$ in $f$ is +-prime,

(ii) Conjecture 1 holds in $L(V \cup\{y\})$,

(iii) Conjecture 2 holds in $\bar{L}(V \cup\{y\})$.

Proof. (i) Proceed by induction on $f(\overrightarrow{2})$; let $f=u(y, \ldots) \cdot \prod_{j} g_{j}(y, \ldots)^{h_{j}(y, \ldots)}$ where $u$ is a monic monomial in $\mathbf{Z}[V \cup\{y\}], f \neq 1, g_{j}, h_{j} \in \bar{L}(V \cup\{y\})-\{1\}$, 
and $\left\{h_{j}\right\}_{j}$ are +-primes. Since $h_{j}(\overrightarrow{2})<f(\overrightarrow{2})$ for all $j$, also $\left\{h_{j}\left(x^{k}, \ldots\right)\right\}_{j}$ are +-primes. Besides, $u\left(x^{k}, \ldots\right)$ is a monic monomial in $\mathbf{Z}[V]$. So by Conjecture 1 for $V$ the function $f\left(x^{k}, \ldots\right)=u\left(x^{k}, \ldots\right) \cdot \prod_{j} g_{j}\left(x^{k}, \ldots\right) \uparrow h_{j}\left(x^{k}, \ldots\right)$ is a t-prime of $\bar{L}(V)$.

(ii) Let $f(y$, other variables $)=u(y, \ldots) \cdot \prod_{j} g_{j}(y, \ldots)^{h_{j}(y, \ldots)}$, where $u$ is a monic monomial in $\mathbf{Z}[V \cup\{y\}], g_{j}, h_{j} \in \bar{L}(V \cup\{y\})-\{1\}$, and $\left\{h_{j}\right\}_{j}$ are +-primes. Then $f(x, \ldots) \in \bar{L}(V)$ is +-prime by (i) and so $f$ itself is +-prime (recall that substituting a function from $\bar{L}(V)$ for $y$ is a $(+, \cdot, \uparrow)$ homomorphism $\bar{L}(V \cup\{y\}) \rightarrow \bar{L}(V))$.

(iii) Let $h_{j}(y$, other variables $) \in \bar{L}(V \cup\{y\}), 1 \leq j \leq m$, be additively prime and $h_{i} \neq h_{j}$ for $i \neq j$. Then for sufficiently large $k \in \mathbf{N}$ we have $h_{i}\left(x^{k}, \ldots\right) \neq h_{j}\left(x^{k}, \ldots\right)$, and $\left\{h_{j}\left(x^{k}, \ldots\right)\right\}_{j}$ are still + -primes by $(\mathrm{i})$. Then by Conjecture 2 for $V:\left\{h_{j}\left(x^{k}, \ldots\right)\right\}_{j}$ are linearly independent over $\mathbf{R}$, whence $\left\{h_{j}\right\}_{j}$ are linearly independent over $\mathbf{R}$.

1.3. Hiromi-Ozawa lemma. This section presents some Borel-type arguments. The Hiromi-Ozawa lemma says that if for some functions $\left\{a_{j}\right\}_{j}$ and $\left\{f_{j}\right\}_{j}$ holomorphic on a disk or on the complex plane $a_{1} \cdot e^{f_{1}}+\cdots+a_{n} \cdot e^{f_{n}}=a_{0}$ and at least one of $e^{f_{1}}, \ldots, e^{f_{n}}$ is Nevanlinna-large as compared to all of $a_{0}, a_{1}, \ldots, a_{n}$ then the functions $a_{1} \cdot e^{f_{1}}, \ldots, a_{n} \cdot e^{f_{n}}$ are linearly dependent over C. This result appeared in [21]; then C. W. Henson and L. A. Rubel in [19] generalized it to functions in several variables (on polydisks and on $\mathbf{C}^{m}$ ) and applied it to Tarski's high school algebra problem. The Hiromi-Ozawa lemma allowed Henson and Rubel to prove some results that are interesting from the logical as well as from the analytical point of view for a class of entire exponential functions (which they call "the Schanuel class") and for a class of "slightly" multivalued exponential functions (which they call "the Levitz class"). However, the typical exponential functions considered in Tarski's high school algebra problem are "wildly" multivalued, and so we sought generalizations of the Hiromi-Ozawa lemma to multivalued exponential functions. And we got some; but instead of "Nevanlinna-large" we use "more multivalued than" criteria; see $\S 4.1$.

In this section we present the Hiromi-Ozawa lemma and several corollaries frequently used in the paper. A terminological remark: A point $\xi$ will be called a zero point of a meromorphic function $f$ if $x(\xi)=0$ for every representation $f=x / y$ with $x$ and $y$ holomorphic in a neighborhood of $\xi$. Similarly, $\xi$ will be called a polar point of $f$ if $y(\xi)=0$ for every representation $f=x / y$ with $x$ and $y$ holomorphic in a neighborhood of $\xi$. A point may be a zero point and a polar point at the same time. This definition will be applied to functions on arbitrary complex-analytic manifolds (not only on $\mathbf{C}^{n}$ ). Sometimes we shall abbreviate "zero point" and "polar point" to "zero" and "pole" respectively.

Lemma 1 (Hiromi-Ozawa lemma). (i) Let $a_{j} \in \mathscr{M}\left(\mathbf{C}^{n}\right), 0 \leq j \leq m$, and $f_{j} \in \mathscr{H}\left(\mathbf{C}^{n}\right), 1 \leq j \leq m$. If

$$
\sum_{j=1}^{m} a_{j} \cdot e^{f_{j}}=a_{0} \quad \text { and } \quad \sum_{j=1}^{m} T\left(r, e^{f_{j}}\right) \neq O\left(\sum_{j=0}^{m} T\left(r, a_{j}\right)\right) \text {, }
$$


where $T(r, g)$ is the Nevanlinna characteristic of $g$, then the functions $\left\{a_{j} \cdot e^{f_{j}}\right\}_{j=1}^{m}$ are linearly dependent over C. (ii) Let $D$ be the unit disk in C. Let $a_{j} \in \mathscr{M}\left(D^{n}\right), 0 \leq j \leq m$, and $f_{j} \in \mathscr{H}\left(D^{n}\right), 1 \leq j \leq m$. If

$$
\sum_{j=1}^{m} a_{j} \cdot e^{f_{j}}=a_{0} \quad \text { and } \quad \sum_{j=1}^{m} T\left(r, e^{f_{j}}\right) \neq O\left(\sum_{j=0}^{m} T\left(r, a_{j}\right)+\log \frac{1}{1-r}\right),
$$

then the functions $\left\{a_{j} \cdot e^{f_{j}}\right\}_{j=1}^{m}$ are linearly dependent over $\mathbf{C}$.

The Hiromi-Ozawa lemma easily implies many results on linear and algebraic (in)dependence of exponentials of holomorphic functions.

Examples. (i) Let $a_{j} \in \mathscr{M}\left(\mathbf{C}^{n}\right)$ and $f_{j} \in \mathscr{H}\left(\mathbf{C}^{n}\right)$ for $1 \leq j \leq m$. If

$$
T\left(r, \sum_{j=1}^{m} a_{j} e^{f_{j}}\right)+\sum_{j=1}^{m} T\left(r, a_{j}\right)=o\left(T\left(r, e^{f_{k}}\right)\right) \quad \text { for all } k=1, \ldots, m,
$$

then $\sum_{j=1}^{m} a_{j} e^{f_{j}}=0$. Indeed, suppose the contrary and take the minimal $m$ for which the statement fails. Then the functions $\left\{a_{j} e^{f_{j}}\right\}_{j}$ are linearly independent over C. Put $a_{0}=\sum_{j=1}^{m} a_{j} e^{f_{j}}$ and apply Lemma 1 .

(ii) Let $\mathscr{L}=\left\{f \in \mathscr{M}\left(\mathbf{C}^{n}\right) \mid \lim \sup \log T(r, f) / \log r<1\right\} \quad(\mathscr{L}$ is the field of functions of the growth order $<1$, all rational functions belong to $\mathscr{L}, \cos \sqrt{z} \in$ $\mathscr{L})$. Let $f_{j} \in \mathscr{H}\left(\mathbf{C}^{n}\right), 1 \leq j \leq m$. If the functions $\left\{e^{f_{j}}\right\}_{j}$ are linearly dependent over $\mathscr{L}$ then $f_{k}-f_{l}=$ const for some $k \neq l$. Indeed, if $\sum_{j=1}^{m} a_{j} e^{f_{j}}=0$ then $\sum_{j=1}^{m-1} a_{j} e^{f_{j}-f_{m}}=-a_{m}$ and hence $a_{m}=0$ by (i), because the order of $e^{f}$ is at least 1 for every nonconstant entire $f$.

(iii) Let $D$ be the unit disk in $\mathrm{C}$ and $\mathscr{L}$ be the subfield of $\mathscr{M}\left(D^{n}\right)$ consisting of functions with Nevanlinna characteristic $O\left(\log \frac{1}{1-r}\right)$. Let $f_{j} \in \mathscr{H}\left(D^{n}\right), 1 \leq$ $j \leq m$. If $T\left(r, e^{f_{k}-f_{l}}\right) \notin \mathscr{L}$ for $k \neq l$ then the functions $\left\{e^{f_{j}}\right\}_{j}$ are linearly independent over $\mathscr{L}$.

To prove this, suppose the contrary and take a minimal subset $A$ of $\left\{e^{f_{j}}\right\}_{j}$ linearly dependent over $\mathscr{L}$. Without loss of generality one can assume that $A=\left\{e^{f_{j}}\right\}_{j=1}^{p}$ for some $p \leq m$. Let $\sum_{j=1}^{p} \lambda_{j} e^{f_{j}}=0$, where $\lambda_{j} \in \mathscr{L}-\{0\}$. Then $\sum_{j=1}^{p-1} \lambda_{j} e^{f_{j}-f_{p}}=-\lambda_{p}$ and by Lemma 1 the functions $\left\{\lambda_{j} e^{f_{j}-f_{p}}\right\}_{j=1}^{p-1}$ are linearly dependent over $\mathbf{C}$. Hence the functions $\left\{e^{f_{j}}\right\}_{j=1}^{p-1}$ are linearly dependent over $\mathscr{L}$ contradicting the minimality of $A$.

(iv) Let $f_{j} \in \mathscr{H}\left(\mathbf{C}^{n}\right), 1 \leq j \leq m$. If $f_{j}(0)=0$ for all $j$ and the functions $\left\{f_{j}\right\}_{j}$ are linearly independent over $\mathbf{Q}$ then the functions $\left\{e^{f_{j}}\right\}_{j}$ are algebraically independent over $\mathbf{C}(z)$. Indeed, a polynomial in $\left\{e^{f_{j}}\right\}_{j}$ with coefficients in $\mathbf{C}(z)$ has the form $\sum_{k=1}^{l} a_{k} e^{p_{k}}$, where $a_{k} \in \mathbf{C}(z)$ and $\left\{p_{k}\right\}_{k}$ are distinct linear combinations of $\left\{f_{j}\right\}_{j}$ with coefficients in $\mathbf{N}_{0}$. Thus the result follows from (ii).

$\S \S 3$ and 4 provide analogues of these statements for multivalued exponential functions, but they are based on measuring the multivaluedness instead of the growth of the functions involved. 
Lemma 2. (i) Let $t: \mathbf{R}_{+} \rightarrow \mathbf{R}_{+}, t(r) \rightarrow \infty$ as $r \rightarrow \infty$. Let $a_{j}, b_{k} \in \mathscr{M}\left(\mathbf{C}^{n}\right)-\{0\}$ and $f_{j}, g_{k} \in \mathscr{H}\left(\mathbf{C}^{n}\right)$ for $1 \leq j \leq p, 1 \leq k \leq q$. Let

$$
\sum_{j=1}^{p} T\left(r, a_{j}\right)+\sum_{k=1}^{q} T\left(r, b_{k}\right)=o(t(r))
$$

and

$$
\underset{j \neq l}{\&} T\left(r, e^{f_{j}-f_{l}}\right) \neq o(t(r)), \quad \underset{k \neq m}{\&} T\left(r, e^{g_{k}-g_{m}}\right) \neq o(t(r)) .
$$

If $\sum_{j=1}^{p} a_{j} e^{f_{j}}=\sum_{k=1}^{q} b_{k} e^{g_{k}}$ then $p=q$ and there is a permutation $\sigma$ of $\{1, \ldots, p\}$ such that $a_{j} e^{f_{j}}=b_{\sigma(j)} \cdot e^{g_{\sigma(j)}}$ for each $j$.

(ii) Let $t:[0,1) \rightarrow \mathbf{R}_{+}$. Let $D$ be the unit disk in $\mathbf{C}$. Let $a_{j}, b_{k} \in \mathscr{M}\left(D^{n}\right)-$ $\{0\}$ and $f_{j}, g_{k} \in \mathscr{H}\left(D^{n}\right)$ for $1 \leq j \leq p, 1 \leq k \leq q$. Let

$$
\sum_{j=1}^{p} T\left(r, a_{j}\right)+\sum_{k=1}^{q} T\left(r, b_{k}\right)+\log \frac{1}{1-r}=o(t(r)),
$$

and

$$
\&_{j \neq l} T\left(r, e^{f_{j}-f_{l}}\right) \neq o(t(r)), \quad \mathbb{k \neq m}_{k \neq m} T\left(r, e^{g_{k}-g_{m}}\right) \neq o(t(r)) .
$$

If $\sum_{j=1}^{p} a_{j} e^{f_{j}}=\sum_{k=1}^{q} b_{k} e^{g_{k}}$ then $p=q$ and there is a permutation $\sigma$ of $\{1, \ldots, p\}$ such that $a_{j} e^{f_{j}}=b_{\sigma(j)} \cdot e^{g_{\sigma(j)}}$ for each $j$.

Proof. Show that each of the families $\left\{a_{j} e^{f_{j}}\right\}_{j}$ and $\left\{b_{k} e^{g_{k}}\right\}_{k}$ is linearly independent over $\mathbf{C}$. Suppose the contrary; let $\left\{a_{j} e^{f_{j}}\right\}_{j}$, say, be linearly dependent over $\mathbf{C}$. Let $\nu \in \mathbf{N}$ be the minimal number for which the functions $\left\{a_{j} e^{f_{j}}\right\}_{j=1}^{\nu+1}$ are linearly dependent over $\mathbf{C}$. Then $\sum_{j=1}^{\nu+1} \lambda_{j} a_{j} e^{f_{j}}=0$ for some $\lambda_{j} \in \mathbf{C}$ with at least one $\lambda_{j} \neq 0$, and hence $\sum_{j=1}^{\nu} \lambda_{j} a_{j} e^{f_{j}-f_{\nu+1}}=-\lambda_{\nu+1} a_{\nu+1}$. By Lemma 1 , the latter identity implies that the functions $\left\{a_{j} e^{f_{j}}\right\}_{j=1}^{\nu}$ are linearly dependent over $\mathbf{C}$, contradicting the choice of $\nu$.

Show that there exist $j$ and $k$ such that $\left(a_{j} e^{f_{j}}\right) /\left(b_{k} e^{g_{k}}\right)=$ const. Recall that the whole family of functions $S=\left\{a_{j} e^{f_{j}}\right\}_{j} \cup\left\{b_{k} e^{g_{k}}\right\}_{k}$ is linearly dependent over C. In fact we shall show more. Let $A$ be a minimal linearly dependent subfamily of $S$ (so that every proper subfamily of $A$ is linearly independent over C)-then $A$ consists of two elements, some $a_{j} e^{f_{j}}$ and some $b_{k} e^{g_{k}}$. Upon a renumbering of the entries one can assume that $A=\left\{a_{j} e^{f_{j}}\right\}_{j=1}^{\mu} \cup\left\{b_{k} e^{g_{k}}\right\}_{k=1}^{\nu}$ for some $\mu \leq p, \nu \leq q$. Both $\mu$ and $\nu$ are $\geq 1$ because both families $\left\{a_{j} e^{f_{j}}\right\}_{j}$ and $\left\{b_{k} e^{g_{k}}\right\}_{k}$ are linearly independent. Let us show that $\mu=\nu=1$. Suppose the contrary, say $\nu>1$. We have $\sum_{j=1}^{\mu} \alpha_{j} a_{j} e^{f_{j}}=\sum_{k=1}^{\nu} \beta_{k} b_{k} e^{g_{k}}$ for some $\alpha_{j}, \beta_{k} \in \mathbf{C}-\{0\}$, and hence

$$
\sum_{j=1}^{\mu} \alpha_{j} a_{j} e^{f_{j}-g_{\nu}}-\sum_{k=1}^{\nu-1} \beta_{k} b_{k} e^{g_{k}-g_{\nu}}-\beta_{\nu} b_{\nu}=0 .
$$

Let $E$ be the set of those summands in $(*)$ above which have Nevanlinna characteristic $\neq o(t(r))$. Note that $E$ does not contain every summand of (*) above since $T\left(r, b_{\nu}\right)=o(t(r))$. On the other hand, $E$ is nonempty since $\beta_{1} b_{1} e^{g_{1}-g_{\nu}} \in E$. By Lemma 1 then $E$ is linearly dependent over $\mathbf{C}$. And a 
linear relation between elements of $E$ upon multiplication by $e^{g_{\nu}}$ becomes a linear relation between elements of $\left\{a_{j} e^{f_{j}}\right\}_{j=1}^{\mu} \cup\left\{b_{k} e^{g_{k}}\right\}_{k=1}^{\nu-1}$, contradicting the choice of $A$.

Note that if $a_{j} e^{f_{j}}=$ const $\cdot b_{k} e^{g_{k}}$ then $\left(a_{k} e^{f_{l}}\right) /\left(b_{k} e^{g_{k}}\right) \neq$ const for $l \neq j$ and $\left(a_{j} e^{f_{j}}\right) /\left(b_{m} e^{g_{m}}\right) \neq$ const for $m \neq k$ (because each of the families $\left\{a_{j} e^{f_{j}}\right\}_{j}$ and $\left\{b_{k} e^{g_{k}}\right\}_{k}$ is linearly independent over $\left.\mathbf{C}\right)$.

Show that for every $j, 1 \leq j \leq p$, there exists a $k$ such that

$$
\left(a_{j} e^{f_{j}}\right) /\left(b_{k} e^{g_{k}}\right)=\text { const . }
$$

Suppose the contrary. Upon a renumbering of entries, one can assume that there exist $\mu, 1 \leq \mu \leq p$, and $\lambda_{j} \in \mathbf{C}-\{0\}, 1 \leq j<\mu$, such that $a_{j} e^{f_{j}}=\lambda_{j} b_{j} e^{g_{j}}$ for $1 \leq j<\mu$ and $\left(a_{j} e^{f_{j}}\right) /\left(b_{k} e^{g_{k}}\right) \neq$ const for $j \geq \mu, k \geq \mu$. Then from $\sum_{j} a_{j} e^{f_{j}}=\sum_{k} b_{k} e^{g_{k}}$ we have

$$
\sum_{j=\mu}^{p} a_{j} e^{f_{j}}=\sum\left\{\left(1-\lambda_{k}\right) b_{k} e^{g_{k}} \mid 1 \leq k \leq q, \quad \lambda_{k} \neq 1\right\}+\sum_{k=\mu}^{q} b_{k} e^{g_{k}} .
$$

The latter identity says that the family of functions $\left\{a_{j} e^{f_{j}}\right\}_{j=\mu}^{p} \cup\left\{b_{k} e^{g_{k}}\right\}_{k=1}^{q}$ is linearly dependent over $\mathbf{C}$ and by the previous paragraph there then exist $j$, $\mu \leq j \leq p$ and $k$ such that $\left(a_{j} e^{f_{j}}\right) /\left(b_{k} e^{g_{k}}\right)=$ const.

Now, by symmetry, for each $k$ there exists a $j$ such that

$$
\left(a_{j} e^{f_{j}}\right) /\left(b_{k} e^{g_{k}}\right)=\text { const . }
$$

Hence $p=q$ and there exist a permutation $\sigma$ of $\{1, \ldots, p\}$ and $\lambda_{j} \in \mathbf{C}-\{0\}$, $1 \leq j \leq p$, such that $b_{\sigma(j)} e^{g_{\sigma(j)}}=\lambda_{j} a_{j} e^{f_{j}}$ for all $j$. Then $\sum_{j=1}^{p}\left(1-\lambda_{j}\right) a_{j} e^{f_{j}}=0$ whereas the functions $\left\{a_{j} e^{f_{j}}\right\}_{j}$ are linearly independent over $\mathbf{C}$. Thus $1-\lambda_{j}=$ 0 for all $j$.

The next corollary is an analogue of the fact that if $r, s \in \mathbf{C}[z]-\{0\}$ and $r / s$ has neither zeros nor poles in $\mathbf{C}^{n}$ then $r$ and $s$ can differ by a constant factor only.

Corollary 3. (i) Let $t: \mathbf{R}_{+} \rightarrow \mathbf{R}_{+}, t(r) \rightarrow \infty$ as $r \rightarrow \infty$. Let $a_{j}, b_{k} \in \mathscr{M}\left(\mathbf{C}^{n}\right)-$ $\{0\}$ and $f_{j}, g_{k} \in \mathscr{H}\left(\mathbf{C}^{n}\right)$ for $1 \leq j \leq p, 1 \leq k \leq q$. Let

$$
\sum_{j=1}^{p} T\left(r, a_{j}\right)+\sum_{k=1}^{q} T\left(r, b_{k}\right)=o(t(r))
$$

and

$$
\underset{j \neq l}{\&} T\left(r, e^{f_{j}-f_{l}}\right) \neq o(t(r)), \quad \underset{k \neq m}{\&} T\left(r, e^{g_{k}-g_{m}}\right) \neq o(t(r)) .
$$

If

$$
h=\frac{\sum_{j=1}^{p} a_{j} e^{f_{j}}}{\sum_{k=1}^{q} b_{k} e^{g_{k}}}
$$

has neither zeros nor poles on $\mathbf{C}^{n}$ or $T(r, h)=o(t(r))$ then $p=q$ and there is a permutation $\sigma$ of $\{1, \ldots, q\}$ such that $h=\left(a_{j} e^{f_{j}}\right) /\left(b_{\sigma(j)} e^{e_{\sigma(j)}}\right)$ for each $j$.

(ii) Let $t:[0,1) \rightarrow \mathbf{R}_{+}$. Let $D$ be the unit disk in $\mathbf{C}$. Let $a_{j}, b_{k} \in \mathscr{M}\left(D^{n}\right)-$ $\{0\}$ and $f_{j}, g_{k} \in \mathscr{H}\left(D^{n}\right)$ for $1 \leq j \leq p, 1 \leq k \leq q$. Let

$$
\sum_{j=1}^{p} T\left(r, a_{j}\right)+\sum_{k=1}^{q} T\left(r, b_{k}\right)+\log \frac{1}{1-r}=o(t(r)),
$$


and

$$
\mathbb{j \neq l}_{j \neq l} T\left(r, e^{f_{j}-f_{l}}\right) \neq o(t(r)), \quad \mathbb{k}_{k \neq m} T\left(r, e^{g_{k}-g_{m}}\right) \neq o(t(r))
$$

If

$$
h=\frac{\sum_{j=1}^{p} a_{j} e^{f_{j}}}{\sum_{k=1}^{q} b_{k} e^{g_{k}}}
$$

has neither zeros nor poles on $D^{n}$ or $T(r, h)=o(t(r))$ then $p=q$ and there exists a permutation $\sigma$ of $\{1, \ldots, p\}$ such that $h=\left(a_{j} e^{f_{j}}\right) /\left(b_{\sigma(j)} e^{g_{\sigma(j)}}\right)$ for all $j$.

Proof. If $h$ has neither zeros nor poles then $h=e^{u}$ for some $u \in \mathscr{H}\left(\mathbf{C}^{n}\right)$ in case (i) or $u \in \mathscr{H}\left(D^{n}\right)$ in case (ii). Then

$$
\sum_{j=1}^{p} a_{j} e^{f_{j}}=\sum_{k=1}^{q} b_{k} e^{g_{k}+u},
$$

and one can apply Lemma 2 to the functions $\left\{a_{j}\right\}_{j},\left\{b_{k}\right\}_{k},\left\{f_{j}\right\}_{j},\left\{g_{k}+u\right\}_{k}$. If $T(r, h)=o(t(r))$ then

$$
\sum_{j=1}^{p} a_{j} e^{f_{j}}=\sum_{k=1}^{q}\left(b_{k} h\right) \cdot d^{g_{k}},
$$

and one can apply Lemma 2 to the functions $\left\{a_{j}\right\}_{j},\left\{b_{k} h\right\}_{k},\left\{f_{j}\right\}_{j},\left\{g_{k}\right\}_{k}$.

Example. (v) Let $a_{j}, b_{k} \in \mathbf{C}(z)-\{0\}$ and $f_{j}, g_{k} \in \mathscr{H}\left(\mathbf{C}^{n}\right)$ for $1 \leq j \leq p$, $1 \leq k \leq q$. Let $f_{j}-f_{l} \neq$ const for $j \neq l$ and $g_{k}-g_{m} \neq$ const for $k \neq m$. If

$$
h=\frac{\sum_{j=1}^{p} a_{j} e^{f_{j}}}{\sum_{k=1}^{q} b_{k} e^{g_{k}}}
$$

has neither zeros nor poles then $p=q$ and there is a permutation $\sigma$ of $\{1, \ldots, p\}$ such that $h=\left(a_{j} e^{f_{j}}\right) /\left(b_{\sigma(j)} e^{\left.g_{\sigma(j)}\right)}\right.$ for all $j$. To prove this, take $t(r)=r$ and apply the above corollary.

Although Nevanlinna theory is not adapted easily to functions meromorphic on $\left(\mathbf{C}_{*}\right)^{n}=(\mathbf{C}-\{0\})^{n}$ instead of $\mathbf{C}^{n}$ (even for $n=1$ ) extensions of Examples (ii), (iv), and (v) are easily available.

Corollary 4. Let $f_{j} \in \mathscr{H}\left(\mathbf{C}_{*}^{n}\right), 1 \leq j \leq m$. If the functions $\left\{e^{f_{j}}\right\}_{j}$ are linearly dependent over $\mathbf{C}(z)$ then $f_{k}-f_{l}=$ const for some $k \neq l$. If for every $\vec{q} \in$ $\mathbf{Q}^{m}-\{0\}$ the linear combination $\sum_{j=1}^{m} q_{j} f_{j} \neq$ const then the functions $\left\{e^{f_{j}}\right\}_{j}$ are algebraically independent over $\mathbf{C}(z)$.

Proof. Consider the first statement. Let $\sum_{j=1}^{m} p_{j} e^{f_{j}}=0$, where $p_{j} \in \mathbf{C}[z]$ and at least one $p_{j} \neq 0$. Let $\left\{\mu_{j t}\right\}_{t}$ be the monomials occurring in $p_{j}$. Put $g_{j}\left(\left\{w_{k}\right\}_{k}\right)=f_{j}\left(\left\{e^{w_{k}}\right\}_{k}\right)$ and $\nu_{j t}\left(\left\{w_{k}\right\}_{k}\right)=\mu_{j t}\left(\left\{e^{w_{k}}\right\}_{k}\right)$. Then $g_{j} \in \mathscr{H}\left(\mathbf{C}^{n}\right)$ and each $\nu_{j t}$ has the form (the coefficient $\lambda_{j t} \in \mathbf{C}$ of $\mu_{j t}$ ) $\exp$ (a linear form $\psi_{j t}$ in $\left.w_{1}, \ldots, w_{n}\right)$. Since $\sum_{j=1}^{m} \sum_{t} \lambda_{j t} \cdot e^{\psi_{j t}+g_{t}}=0$, there exist $(j, t) \neq(k, u)$ such that $\psi_{j t}+g_{j}-\psi_{k u}-g_{k}=$ const, or $\psi_{j t}-\psi_{k u}=g_{k}-g_{j}+$ const. Since the righthand side of the latter identity is periodic in each of $\left\{w_{l}\right\}_{l}$ while the left-hand side is a linear form, $\psi_{j t}=\psi_{k u}$ and $g_{k}=g_{j}+$ const. Hence $f_{j}-f_{k}=$ const.

The second statement of this corollary follows from the first one by the same reasoning as that used in Example (iv). 
Corollary 5. Let $a_{j} \in \mathbf{C}(z)-\{0\}, f_{j} \in \mathscr{H}\left(\mathbf{C}_{*}^{n}\right)$ for $1 \leq j \leq p$, and $b_{k} \in$ $\mathbf{C}(z)-\{0\}, g_{k} \in \mathscr{H}\left(\mathbf{C}_{*}^{n}\right)$ for $1 \leq k \leq q$. Let $f_{j}-f_{j^{\prime}} \neq$ const for $j \neq j^{\prime}$ and $g_{k}-g_{k^{\prime}} \neq$ const for $k \neq k^{\prime}$. If

$$
h=\frac{\sum_{j=1}^{p} a_{j} e^{f_{j}}}{\sum_{k=1}^{q} b_{k} e^{g_{k}}}
$$

has neither zeros nor poles in $\mathbf{C}_{*}^{n}$ then $p=q$ and there is a permutation $\sigma$ of $\{1, \ldots, q\}$ such that $h=\left(a_{j} / b_{\sigma(j)}\right) \cdot e^{f_{j}-g_{\sigma(j)}}$ for each $j$.

Proof. Without loss of generality one can assume that $a_{j}, b_{k} \in \mathbf{C}[z]$. Let $\left\{\mu_{j t}\right\}_{t}$ be the monomials occurring in $a_{j}$ and $\left\{\nu_{k u}\right\}_{u}$ be the monomials occurring in $b_{k}$. Put $\hat{r}(w)=r\left(\left\{e^{w_{l}}\right\}_{l}\right)$ for each function $r$ occurring. Then $\hat{h}, \hat{f}_{j}, \hat{g}_{k} \in$ $\mathscr{H}\left(\mathbf{C}^{n}\right)$ and $\hat{\mu}_{j t}=\alpha_{j t} \cdot e^{\varphi_{j t}}, \hat{\nu}_{k u}=\beta_{k u} \cdot e^{\psi_{k u}}$, where $\alpha_{j t}, \beta_{k u} \in \mathbf{C}_{*}$ and $\varphi_{j t}, \psi_{k u}$ are linear forms in $w_{1}, \ldots, w_{n}$. Note that $\varphi_{j t}-\varphi_{j^{\prime} t^{\prime}}+\hat{f}_{j}-\hat{f}_{j^{\prime}} \neq$ const unless $(j, t)=\left(j^{\prime}, t^{\prime}\right)$ and $\psi_{k u}-\psi_{k^{\prime} u^{\prime}}+\hat{g}_{k}-\hat{g}_{k^{\prime}} \neq$ const unless $(k, u)=\left(k^{\prime}, u^{\prime}\right)$. By Corollary 3 the set of $(j, t)$ pairs and the set of $(k, u)$ pairs have the same cardinality and there exists a bijection $\tau$ between them such that $\hat{h}=\left(\alpha_{j t} / \beta_{k u}\right)$. $\exp \left(\varphi_{j t}-\psi_{k u}+\hat{f}_{j}-\hat{g}_{k}\right)$ for $(k, u)=\tau(j, t)$. Hence $h=\left(\mu_{j t} / \nu_{k u}\right) \cdot e^{f_{j}-g_{k}}$ for $(k, u)=\tau(j, t)$.

Let us show that $k$ in $(k, u)=\tau(j, t)$ depends on $j$ only (does not depend on $t)$. Suppose $\left(k^{\prime}, u^{\prime}\right)=\tau\left(j, t^{\prime}\right)$. Then $h=\left(\mu_{j t} / \nu_{k u}\right) \cdot e^{f_{j}-g_{k}}=\left(\mu_{j t^{\prime}} / \nu_{k^{\prime} u^{\prime}}\right) \cdot$ $e^{f_{j}-g_{k^{\prime}}}$ and hence $e^{g_{k}-g_{k^{\prime}}}=\left(\mu_{j t} \nu_{k^{\prime} u^{\prime}}\right) /\left(\nu_{k u} \mu_{j t^{\prime}}\right)$. However, $e^{g_{k}-g_{k^{\prime}}}$ is not equal to a quotient of monomials unless $k=k^{\prime}$. Symmetrically, $j$ in $(j, t)=$ $\tau^{-1}(k, u)$ depends on $k$ only. Consequently $p=q$ and $\sigma: j \mapsto \tau(j$, any $t)$ is a permutation of $\{1, \ldots, p\}$. Further, $\tau$ induces a bijection between $\left\{\mu_{j t}\right\}_{t}$ and $\left\{\nu_{k u}\right\}_{u}$ for $k=\sigma(j)$, and $h=\left(\mu_{j t} / \nu_{\tau(j, t)}\right) \cdot e^{f_{j}-g_{\sigma(j)}}$ implies $\mu_{j t} / \nu_{\tau(j, t)}=$ $\mu_{j t^{\prime}} / \nu_{\tau\left(j, t^{\prime}\right)}$. Thus $\mu_{j t} / \nu_{\tau(j, t)}=a_{j} / b_{\sigma(j)}$.

The idea in the proofs of Corollaries 4 and 5 will be further exploited in the next subsection and in $\S 2$.

1.4. Some applications to Tarski's high school algebra problem. In this subsection we explain how the Hiromi-Ozawa lemma is applied to the Schanuel class $\Sigma$ (mostly following Henson and Rubel [19]) and to the Levitz class $\Lambda$ (much simpler than in [19]). Both $\bar{\Sigma}$ and $\bar{\Lambda}$ happen to be free semirings ${ }^{5}$ with units, and their systems of generators are easy to describe. This fact allows one to prove easily that Tarski's conjecture holds for $\Sigma$ and $\Lambda$.

Recall that $\Sigma=\{t \in L \mid$ if $a \uparrow b$ occurs as a subterm of $t$ then $a$ contains no variables $\}$. Elements of $\bar{\Sigma}$ are entire functions. By Corollary 3 of $\S 1.2$ each $f \in \bar{\Sigma}$ is a sum of +-primes of $\bar{\Sigma}$ and every +-prime $f$ of $\bar{\Sigma}$ can be presented

\footnotetext{
${ }^{5} \mathrm{~A}$ semiring is an algebraic system with binary operations + and $\cdot$ which are commutative, associative, and satisfy the distributivity law $x \cdot(y+z)=x \cdot y+x \cdot z$. In this paper "semiring" will mean a semiring with the unit, i.e., containing an element 1 satisfying $1 \cdot x=x$. For a semiring $S$ with unit and $X \subset S-\{1\}$ it is said that $S$ is free over $X$ if $S$ is generated by $X \cup\{1\}$ and the elements of $X$ are algebraically independent, i.e., for all polynomials $P\left(\left\{t_{i}\right\}_{i}\right), Q\left(\left\{t_{i}\right\}_{i}\right)$ with positive integer coefficients and every $x_{i} \in X$ if $P\left(\left\{x_{i}\right\}_{i}\right)=Q\left(\left\{x_{i}\right\}_{i}\right)$ then $P=Q$. Obviously, if $S$ is free over $X$ then $X$ is the set of $(+, \cdot)$-primes of $S$ and $S$ is isomorphic to the semiring of polynomials with positive integer coefficients in the indeterminates corresponding to the elements of $X$.
} 
in the form

(a monic monomial)

$$
\times \prod_{j}(\text { a number } \in \mathbf{N}-\{1\})_{j} \uparrow(\mathbf{a}+\text {-prime } \in-\Sigma, \neq 1)_{j},
$$

perhaps with empty $\prod_{j}$, with all the exponents occurring in $\prod_{j}$ distinct.

Proposition 1. (i) Let $f, g \in \bar{\Sigma}$ possess representations of the form $\left(\nabla_{0}\right)$. If $f / g=$ const then $f=g$ and their $\left(\nabla_{0}\right)$ representations coincide up to a permutation of factors.

(ii) Let $\left\{f_{k}\right\}_{k} \in \bar{\Sigma}$ have the form $\left(\nabla_{0}\right)$; if $f_{i} \neq f_{j}$ for $i \neq j$ then $\left\{f_{k}\right\}_{k}$ are linearly independent over $\mathbf{R}$.

An immediate consequence of this proposition is that every function which can be presented in the form $\left(\nabla_{0}\right)$ is +-prime. Indeed, the statement that such a function is a sum of several others of the $\left(Q_{0}\right)$ form is a linear relation.

Proof. Proceed by induction on the values at $\overrightarrow{2}$ of the functions occurring, viz. let $M \in \mathbf{N}$ and assume that (i) holds whenever all exponents occurring in $\left(\nabla_{0}\right)$ have values $<M$ at $\overrightarrow{2}$, and that (ii) holds whenever $\max \left\{f_{k}(\overrightarrow{2})\right\}_{k}<M$. Consider the induction steps.

$\left(\mathrm{i}_{M+1}\right) \quad f=\mu \cdot e^{\varphi}$ and $g=\nu \cdot e^{\psi}$ for some monic monomials $\mu, \nu$ and entire functions $\varphi, \psi$. Then $(\mu / \nu) \cdot e^{\varphi-\psi}=$ const and consequently $\mu=\nu$. Thus one can assume that $f$ and $g$ have no monomial factors in the $\left(\nabla_{0}\right)$ form. Since the values at integral points of functions from $\bar{\Sigma}$ are integral, $f / g=m / n$ with some $m, n \in \mathbf{N}$. Let $\alpha \in \mathbf{N}$ be a prime and

$$
f=\prod_{i}\left(p_{i}\right)^{r_{i}} \quad \text { and } \quad g=\prod_{j}\left(q_{j}\right)^{s_{j}},
$$

in the $\left(Q_{0}\right)$ form. Consider the degrees with which $\alpha$ occurs in the sides of the identity $n f=m g$. Let $m=\alpha^{\mu} \cdot m^{\prime}, n=\alpha^{\nu} \cdot n^{\prime}, p_{i}=\alpha^{k_{i}} \cdot p_{i}^{\prime}$, $q_{j}=\alpha^{l_{j}} \cdot q_{j}^{\prime}$, where $\mu, m^{\prime}, \nu, n^{\prime}, k_{i}, p_{i}^{\prime}, l_{j}, q_{j}^{\prime}$ are nonnegative integers and all of $m^{\prime}, n^{\prime}, p_{i}^{\prime}, q_{j}^{\prime}$ are not divisible by $\alpha$. Then

$$
\nu+\sum_{i} k_{i} r_{i}=\mu+\sum_{j} l_{j} s_{j} .
$$

Since all $r_{i}(\overrightarrow{2})$ and $s_{j}(\overrightarrow{2})$ are $<M+1$ one can apply $\left(\mathrm{ii}_{M}\right)$ to $1, r_{i}$ and $s_{j}$. The above identity $(*)$ is a linear relation between $1, r_{i}$ and $s_{j}$, and hence the sides of $(*)$ must coincide up to a permutation of summands. (Recall that $r_{i} \neq r_{i^{\prime}}$ for $i \neq i^{\prime}$ and $s_{j} \neq s_{j^{\prime}}$ for $j \neq j^{\prime}$.) Consequently $\mu=\nu$ and there exists a bijection $\sigma_{\alpha}$ from $I_{\alpha}=\left\{i \mid k_{i} \neq 0\right\}$ to $J_{\alpha}=\left\{j \mid l_{j} \neq 0\right\}$ such that $r_{i}=s_{\sigma_{\alpha}(i)}$ and $k_{i}=l_{\sigma_{\alpha}(i)}$. Note that $\sigma_{\alpha}$ is determined uniquely.

Since $\alpha$ is arbitrary, the identity $\mu=\nu$ implies that $m=n$ (they have the same decomposition into primes). Put $\sigma=\bigcup_{\alpha} \sigma_{\alpha}$. Since $p_{i} \neq 1$ for each $i$ there exists a prime $\alpha$ such that $p_{i}$ is divisible by $\alpha, p_{i}=\alpha^{k_{i}} \cdot p_{i}^{\prime}, k_{i}, p_{i}^{\prime} \in$ $\mathbf{N}$. Thus each $i$ belongs to the domain of some $\sigma_{\alpha}$. Symmetrically, each $j$ belongs to the range of some $\sigma_{\alpha}$. Since $r_{i}$ 's are distinct and $s_{j}$ 's are distinct, if $i \in \operatorname{dom}\left(\sigma_{\alpha}\right) \cap \operatorname{dom}\left(\sigma_{\beta}\right)$ then $\sigma_{\alpha}(i)=\sigma_{\beta}(i)$-because both are the number $j$ 
for which $s_{j}=r_{i}$. Thus $\sigma$ is a bijection of the set of $i$-indices to the set of $j$ indices. Now the identity $k_{i}=l_{\sigma(i)}$ implies that $p_{i}=q_{\sigma(i)}$ (they have the same decomposition into primes) for each $i$. Thus $\sigma$ is the required permutation of factors transforming the $\left(\nabla_{0}\right)$ form of $f$ into that of $g$.

$\left(\mathrm{ii}_{M+1}\right)$ Suppose that $\left\{f_{i}\right\}_{i}$ are linearly dependent over $\mathbf{R}$. Then by the Hiromi-Ozawa lemma (see $\S 1.3$ ) there are $i \neq j$ such that $f_{i} / f_{j}=$ const and then $f_{i}=f_{j}$ by $\left(\mathrm{i}_{M+1}\right)$.

The corollary below is essentially a reformulation of Proposition 1.

Corollary 2. $\bar{\Sigma}$ is a free semiring with unit whose system of generators (the $(+, \cdot)$-primes of $\bar{\Sigma}$ sans 1) is: $\{$ the variables $\} \cup\left\{p^{f} \mid p\right.$ a prime in $\mathbf{N}$ and $f$ $a$ t-prime in $\bar{\Sigma}, f \neq 1\}$.

Proof. We need to show that the above list is indeed the list of all $(+, \cdot)$-primes of $\bar{\Sigma}$ and that they are algebraically independent.

Each $(+, \cdot)$-prime $t \neq 1$ of $\bar{\Sigma}$, as a + -prime, can be presented in the form $\left(\nabla_{0}\right)$, and (as a multiplicative prime) must have only one factor $\neq 1$ in it. If that is the "monic monomial" factor then $t$ is a variable. Otherwise $t$ is $p^{u}$, where $p \in \mathbf{N}-\{1\}$ and $u$ is a +-prime of $\bar{\Sigma}, u \neq 1$. Then $p$ must be a prime. Thus all $(+, \cdot)$-primes of $\bar{\Sigma}$ are contained in the list presented, and Proposition 1(i) makes sure that a +-prime can be decomposed into a product of $(+, \cdot)$-primes in only one way.

An algebraic dependence between $(+, \cdot)$-primes $\left\{t_{i}\right\}_{i=1}^{n}$ is a linear relation between some monomials in them, $t^{\alpha}=\prod_{i=1}^{n}\left(t_{i}\right)^{\alpha_{i}}, \alpha \in\left(\mathbf{N}_{0}\right)^{n}$. Since each $t^{\alpha}$ can be presented in the form $\left(\nabla_{0}\right)$, Proposition 1 (ii) implies that $t^{\alpha}=t^{\beta}$ for some $\alpha, \beta \in\left(\mathbf{N}_{0}\right)^{n}, \alpha \neq \beta$. But the latter is prohibited by Proposition 1(i) when all $t_{i} \neq 1$.

Recall that $\Lambda=\{t \in L \mid$ if $a \uparrow b$ occurs as a subterm of $t$ then either $a$ is a variable or $a$ contains no variables at all $\}. \Sigma \subset \Lambda$ and $\bar{\Lambda}$ is strictly larger than $\bar{\Sigma}$. For example $x^{y} \in \Lambda$ and the function it represents is not entire, whence not in $\bar{\Sigma}$. By Corollary 3 of $\S 1.12$ each $f \in \bar{\Lambda}$ is a sum of +-primes of $\bar{\Lambda}$ and every +-prime of $\bar{\Lambda}$ can be presented in the form

(a monic monomial)

$$
\times \prod_{j}(\text { a monomial }, \neq 1)_{j} \uparrow(\mathrm{a}+\text {-prime } \in \bar{\Lambda}, \neq 1),
$$

perhaps with empty $\prod_{j}$, with all exponents occurring in $\Pi_{j}$ distinct. Consequently the $(+, \cdot)$-primes of $\bar{\Lambda}$ are contained in the set $\{1\} \cup\{$ the variables $\} \cup\left\{p^{f} \mid p\right.$ a prime in $\mathbf{N}$ and $f$ a + -prime in $\left.\bar{\Lambda}, f \neq 1\right\} \cup\left\{x^{f} \mid x\right.$ a variable and $f$ a +-prime in $\bar{\Lambda}\}$.

Although $\bar{\Lambda}$ contains multivalued functions, it is still very close to $\bar{\Sigma}$. Consider the transformation $\chi: \bar{\Lambda} \rightarrow \bar{\Lambda}$ given by

$$
f\left(\left\{x_{i}\right\}_{i}\right) \mapsto f\left(\left\{2^{x_{i}}\right\}_{i}\right) .
$$

Note that $\chi$ preserves operations $+, \cdot, \uparrow$ and $\chi(f) \neq \chi(g)$ whenever $f \neq g$. Consequently, if $\chi(f)$ is $\$$-prime then $f$ is $\$$-prime, $\$ \in\{+, \cdot, \uparrow\}$ (if $f=$ $g \$ h$ and $f \neq g, f \neq h$ then $\chi(f)=\chi(g) \$ \chi(h)$ and $\chi(f) \neq \chi(g), \chi(f) \neq$ 
$\chi(h))$. It turns out that $\chi$ transforms $\bar{\Lambda}$ into a subset of $\bar{\Sigma}$ and in a very neat way.

Proposition 3. $\chi\{$ the $(+, \cdot)$-primes of $\bar{\Lambda}\} \subset\{$ the $(+, \cdot)$-primes of $\bar{\Sigma}\}$ and $\chi\{$ the + -primes of $\bar{\Lambda}\} \subset\{$ the + -primes of $\bar{\Sigma}\} \cap$ exponentials of entire functions $\}$.

Proof. Proceed by induction on the values at $\overrightarrow{2}$ of the functions occurring. The induction base, $f(\overrightarrow{2})=1$, is trivial in view of Proposition 2 of $\S 1.2$. Let $M \in \mathbf{N}$ and assume that $\chi(f)$ is a $(+, \cdot)$-prime of $\bar{\Sigma}$ for any $(+, \cdot)$-prime $f$ of $\bar{\Lambda}$ with $f(\overrightarrow{2})<M$, and that $\chi(f)$ is an exponential of an entire function and a +-prime of $\bar{\Sigma}$ for any +-prime $f$ of $\bar{\Lambda}$ with $f(\overrightarrow{2})<M$. Consider the induction steps.

Let $f$ be a $(+, \cdot)$-prime of $\bar{\Lambda}$ and $f(\overrightarrow{2}) \leq M$. If $f$ is a variable $v$ then $\chi(f)=2^{v} \in \bar{\Sigma}$ and is a $(+, \cdot)$-prime by Corollary 2. Consider $f=p^{g}$, where $p$ is a prime in $\mathbf{N}$ and $g$ is a t-prime of $\bar{\Lambda}$. Then $\chi(f)=p^{\chi(g)}$ and $g(\overrightarrow{2})<M$. By the induction assumption $\chi(g)$ is a +-prime of $\bar{\Sigma}$ and then $\chi(f)$ is a $(+, \cdot)$-prime of $\bar{\Lambda}$ by Corollary 2. Consider $f=v^{g}$, where $v$ is a variable and $g$ is a +-prime of $\bar{\Lambda}$. Then $\chi(f)=2^{v \cdot \chi(g)}$ and $g(\overrightarrow{2})<M$. By the induction assumption $\chi(g)$ is a +-prime of $\bar{\Sigma}$ and by Corollary 2 then $v \cdot \chi(g)$ is a +-prime of $\bar{\Sigma}$, whence $\chi(f)$ is a $(+, \cdot)$-prime of $\bar{\Sigma}$ again by Corollary 2 .

Let $f$ be a +-prime but not a $(+, \cdot)$-prime of $\bar{\Lambda}$. Then $f$ is a product of $(+, \cdot)$-primes of $\bar{\Lambda}$ by Corollary 3 of $\S 1.2$. Each of those has value $<M$ at $\overrightarrow{2}$ and by the induction assumption is transformed by $\chi$ into a $(+, \cdot)$-prime of $\bar{\Sigma}$. By Corollary 2 then $\chi(f)$ is a +-prime of $\bar{\Sigma}$.

The above proposition easily implies the following.

Corollary 4. (i) Let $f$ and $g$ possess representations of the form $\left(\boldsymbol{\alpha}_{0}\right)$. If $f / g=$ const then $f=g$ and their $\left(\boldsymbol{\$}_{0}\right)$ representations coincide up to a permutation of factors.

(ii) Let $\left\{f_{k}\right\}_{k} \subset \bar{\Lambda}$ have the form $\left(\boldsymbol{n}_{0}\right)$; if $f_{i} \neq f_{j}$ for $i \neq j$ then $\left\{f_{k}\right\}_{k}$ are linearly independent over $\mathbf{R}$.

(iii) $\bar{\Lambda}$ is a free semiring with unit, whose system of generators $($ the $(+, \cdot)$ primes of $\bar{\Lambda}$ sans 1) is: \{the variables $\} \cup\left\{p^{f} \mid p\right.$ a prime in $\mathbf{N}$ and $f$ a +-prime of $\bar{\Lambda}, f \neq 1\} \cup\left\{v^{f} \mid v\right.$ a variable and $f$ a +-prime of $\left.\bar{\Lambda}, f \neq 1\right\}$.

Proof. (i) Let

$$
f=\mu_{0} \cdot \prod_{i=1}^{m}\left(\mu_{i}\right)^{p_{i}} \quad \text { and } \quad g=\nu_{0} \cdot \prod_{j=1}^{n}\left(\nu_{j}\right)^{q_{j}}
$$

according to $\left(\boldsymbol{m}_{0}\right)$, and $f / g=$ const. Consider $\chi(f)$ and $\chi(g)$. Since they are +-primes of $\bar{\Sigma}$ and $\chi(f) / \chi(g)=$ const, we have $\chi(f)=\chi(g)$ and hence $f=g$. Let $\left\{x_{k}\right\}_{k}$ be the variables occurring in $f$ and $g$. Let $\mu_{0}=\prod_{k}\left(x_{k}\right)^{r_{k}}$, $\mu_{i}=a_{i} \cdot \prod_{k}\left(x_{k}\right)^{s_{k}}, \nu_{0}=\prod_{k}\left(x_{k}\right)^{t_{k}}, \nu_{j}=b_{j} \cdot \prod_{k}\left(x_{k}\right)^{u_{k}}$, where $r_{k}, s_{k}, t_{k}, u_{k} \in \mathbf{N}_{0}$ and $a_{i}, b_{j} \in \mathbf{N}$. Then

$$
\chi(f)=\prod\left\{\left(2^{r_{k}}\right)^{x_{k}} \mid r_{k} \neq 0\right\} \cdot \prod\left\{\left(a_{i}\right)^{\chi\left(p_{i}\right)} \mid a_{i} \neq 1\right\} \cdot \prod\left\{\left(2^{s_{k}}\right)^{x_{k} \cdot \chi\left(p_{i}\right)} \mid s_{k} \neq 0, i\right\}
$$

and

$$
\chi(g)=\prod\left\{\left(2^{t_{k}}\right)^{x_{k}} \mid t_{k} \neq 0\right\} \cdot \prod\left\{\left(b_{j}\right)^{\chi\left(q_{j}\right)} \mid b_{j} \neq 1\right\} \cdot \prod\left\{\left(2^{u_{k}}\right)^{x_{k} \cdot x\left(q_{j}\right)} \mid u_{k} \neq 0, j\right\}
$$


in the $\left(\nabla_{0}\right)$ form with the trivial (i.e., empty) "monic monomial" factors.

To prove this, show that $x_{k} \neq \chi(c), x_{k} \neq x_{l} \cdot \chi(c), \chi(c) \neq x_{k} \cdot \chi(d)$, and $x_{k} \cdot \chi(c) \neq x_{l} \cdot \chi(d)$ unless $k=l$ and $c=d$, for any +-primes $c, d$ of $\bar{\Lambda}$, $c \neq 1, d \neq 1$. Identity $x_{k}=\chi(c)$ is impossible because $\chi(c)$ is an exponential of an entire function while $x_{k}$ is not. Identity $x_{k}=x_{l} \cdot \chi(c)$ is equivalent to $x_{k} / x_{l}=\chi(c)$. The right-hand side of the latter identity is an exponential of an entire function and consequently $x_{k} / x_{l}$ is an exponential of an entire function. Hence $k=l$ and $\chi(c)=1$, whereas $c \neq 1$ (a contradiction). Identity $\chi(c)=x_{k} \cdot \chi(d)$ is impossible because $\chi(c) / \chi(d)$ is an exponential of an entire function while $x_{k}$ is not. Identity $x_{k} \cdot \chi(c)=x_{l} \cdot \chi(d)$ is equivalent to $x_{k} / x_{l}=\chi(d) / \chi(c)$. The right-hand side of the latter identity is an exponential of an entire function and consequently $x_{k} / x_{l}$ must be an exponential of an entire function. Thus $k=l$ and $\chi(c)=\chi(d)$, whence $c=d$.

By Proposition 1, the above representations of $\chi(f)$ and $\chi(g)$ must coincide up to a permutation of factors. In view of the above remarks, such a permutation must send the factors of each of the products $\Pi\{\cdots\}$ in the representation of $\chi(f)$ above into the factors of the corresponding $\Pi\{\cdots\}$ of the representation of $\chi(g)$. Consequently $r_{k}=t_{k}$ for each $k$ (the bases corresponding to the exponent $x_{k}$ must coincide), $m=n$ (the number of exponentials in the second $\prod\{\cdots\}$ must coincide), there exists a permutation $\sigma$ of $\{1, \ldots, m\}$ such that $\chi\left(p_{i}\right)=\chi\left(q_{\sigma(i)}\right)$ and hence $p_{i}=q_{\sigma(i)}$ for each $i$ (the sets of exponents in the second $\prod\{\cdots\}$ must coincide), $a_{i}=b_{\sigma(i)}$ for each $i$ (the bases corresponding to the exponent $\chi\left(p_{i}\right)=\chi\left(q_{\sigma(i)}\right)$ must coincide), and $s_{k}=u_{k}$ for each $k$ (the bases corresponding to the exponent $x_{k} \cdot \chi\left(p_{i}\right)=x_{k} \cdot \chi\left(q_{\sigma(i)}\right)$ must coincide). Hence the $\left(\boldsymbol{\beta}_{0}\right)$ representations of $f$ and $g$ coincide up to a permutation of factors.

(ii) Let $a_{k} \in \mathbf{R}$ and $\sum_{k} a_{k} f_{k}=0$. Then $\sum_{k} a_{k} \cdot \chi\left(f_{k}\right)=0$, and each $\chi\left(f_{k}\right)$ can be presented in a $\left(Q_{0}\right)$ form. By Proposition 1 , all $a_{k}=0$ or there exist $k \neq l$ such that $\chi\left(f_{k}\right)=\chi\left(f_{l}\right)$. But the latter would imply $f_{k}=f_{l}$.

(iii) Together (i) and (ii) obviously imply that the $(+, \cdot)$-primes of $\bar{\Lambda}$ have the forms shown, and that the $(+, \cdot)$-primes of $\bar{\Lambda}$ are algebraically independent.

Proposition 5. Let $f, g \in \bar{\Sigma}$ both have content $=1$. If $f / g$ has neither zeros nor poles on $\mathbf{C}^{n}$ ( $n=$ the number of variables occurring) then $f=g$.

Proof. Let us regard the polynomials in the $(+, \cdot)$-primes of $\bar{\Sigma}$ (sans 1) as elements of $\left(\mathbf{N}_{0}[\vec{x}]\right)[\vec{X}]$, where the lower case formal variables are the actual variables occurring and the upper case formula variables stand for the $(+, \cdot)$ primes of $\bar{\Sigma}$ of the form (a prime $\in \mathbf{N}) \uparrow(a+$-prime of $\bar{\Sigma}, \neq 1)$. Each monic monomial in $\vec{X}$ is an exponential of an entire function on some $\mathbf{C}^{n}$ and each polynomial in $\vec{x}$ is indeed a polynomial function on some $\mathbf{C}^{n}$. Recall that the functions represented by distinct monic monomials in $\vec{X}$ are linearly independent over $\mathbf{R}$ (the $(+, \cdot)$-primes of $\bar{\Sigma}$ sans 1 are algebraically independent). For a multi-index $\gamma=\left(\gamma_{1}, \ldots, \gamma_{k}\right)$ denote $X^{\gamma}=\prod_{i}\left(X_{i}\right)^{\gamma_{i}}$.

Let $f=\sum_{\alpha \in A} p_{\alpha} X^{\alpha}$ and $g=\sum_{\beta \in B} q_{\beta} X^{\beta}$, where $p_{\alpha}, q_{\beta} \in \mathbf{N}_{0}[\vec{x}]-\{0\}$ and $A, B$ are some finite sets of multi-indices. Then by Corollary 4 of $\S 1.3$ the number of elements in $A$ and $B$ are the same and there exists a bijection $\sigma: A \rightarrow B$ such that $f / g=\left(p_{\alpha} / q_{\sigma(\alpha)}\right) \cdot X^{\alpha-\sigma(\alpha)}$ for each $\alpha \in A$. Again, since $f / g$ has neither poles nor zeros, each $p_{\alpha} / q_{\sigma(\alpha)}$ has neither poles nor zeros and 
hence is a constant. Thus $f / g=$ const $_{\alpha} \cdot X^{\alpha-\sigma(\alpha)}$ for all $\alpha \in A$. Since $\left\{X^{\gamma}\right\}_{\gamma}$ are linearly independent, all $\alpha-\sigma(\alpha)$ coincide. Denote $\theta=\alpha-\sigma(\alpha), \theta$ not depending on $\alpha$. Let us show that $\theta=\overrightarrow{0}$ (then we will have $\sigma(\alpha)=\alpha$ for all $\alpha)$. Note that $\gamma_{i} \geq 0,1 \leq i \leq k$, for each $\gamma=\left(\gamma_{1}, \ldots, \gamma_{k}\right) \in A \cup B$. Let $1 \leq j \leq k$. Since the content of $f$ is 1 , there exists an $\alpha=\left(\alpha_{1}, \ldots, \alpha_{k}\right) \in A$ with $\alpha_{j}=0$. Hence $\theta_{j}=(\alpha-\sigma(\alpha))_{j}=-(\sigma(\alpha))_{j} \leq 0$. Since the content of $g$ is 1 , there exists a $\beta=\left(\beta_{1}, \ldots, \beta_{k}\right) \in B$ with $\beta_{j}=0$. Hence $\theta_{j}=$ $\left(\sigma^{-1}(\beta)-\beta\right)_{j}=\left(\sigma^{-1}(\beta)\right)_{j} \geq 0$. Thus $A=B$ and $f=g=p_{\alpha} / q_{\alpha}=$ const $\in \mathbf{Q}$ for all $\alpha \in A$. Let $f / g=l / m$, where $l, m \in \mathbf{N}$ are mutually prime. Then each $p_{\alpha}$ is divisible by $l$, contradicting content $(f)=1$ unless $l=1$. Similarly, $m=1$.

The following is an easy consequence of Proposition 5.

Corollary 6. Let $f, g \in \bar{\Lambda}$ both have content $=1$. If for the analytic continuations of $f$ and $g$ along any path in $\left(\mathbf{C}_{*}\right)^{n}$ beginning in $\left(\mathbf{R}_{+}\right)^{n}$ the quotients $f / g$ have neither zeros nor poles then $f=g$.

Proof. By Proposition 3 then $\chi(f), \chi(g) \in \bar{\Sigma}$ and both have content $=1$. Besides, $\chi(f) / \chi(g)$ has neither zeros nor poles on $\mathbf{C}^{n}$, and by the above proposition then $\chi(f)=\chi(g)$. Hence $f=g$.

Corollary 7. Tarski's conjecture holds for $\Lambda$, i.e., if $t, u \in \Lambda$ and $\bar{t}=\bar{u}$ (in other words, $\mathbf{R}_{+} \vDash t=u$ ) then $\mathscr{T} \vdash t=u$.

Proof. Let us show that each term $t$ in $\Lambda$ is $\mathscr{T}$-equivalent to a term of the form

$$
\sum_{i}\left(\prod_{j} t_{i j}\right)
$$

there $t_{i j} \in \Lambda$ and all $\overline{t_{i j}}$ are $(+, \cdot)$-primes in $\bar{\Lambda}$ (the sum may consist of one summand only, any product may consist of one factor only).

Proceed by induction on the construction of $t$. If $t$ is a constant symbol then $t=g 1+\cdots+1$. If $t$ is a variable symbol then it is already in the required form. Case $t=u+v$ is trivial. In case $t=u \cdot v$ apply the distributivity law to multiply the representations of $u$ and $v$. Consider $t=u \uparrow v$, let $v$ be presented as required: $v=\sum_{i=1}^{n}\left(\prod_{j} v_{i j}\right)$ with $v_{i j} \in \Lambda$ and $\overline{v_{i j}}(+, \cdot)$-prime in $\bar{\Lambda}$. To obtain a required representation of $t$ proceed by induction on $n$. Note that $u$ is either a constant or a variable symbol.

Let $n=1$. If $u$ is a variable symbol then $\overline{u \uparrow v}$ is $(+, \cdot)$-prime and thus has the required form. If $u$ is a constant symbol and $\bar{u}=1$ then $t=\mathscr{T} 1$. If $u$ is a constant symbol and $\bar{u} \neq 1$ then decompose $\bar{u}$ into a product of primes $\prod_{l} p_{l}$ in $\mathbf{N}$; then $t=g \prod_{l}\left(p_{l} \uparrow v\right)$ with the right-hand side having the required form. Let $n>1$. Denote $v_{i}=\prod_{j} v_{i j}$. Then $t=\mathscr{g}\left(u \uparrow v_{n}\right) \cdot\left(u \uparrow v^{\prime}\right)$, where $v^{\prime}=\sum_{i=1}^{n-1}$, and each factor is $\mathscr{T}$-equivalent to a term of the required form. It remains then to apply the distributivity law to multiply the representations of the factors.

Now let $t, u \in \Lambda$; present both in the above form:

$$
t=\mathscr{g} \sum_{i}\left(\prod_{j} t_{i j}\right) \quad \text { and } u=\mathscr{T} \sum_{i}\left(\prod_{j} u_{i j}\right) \text {, }
$$


with $t_{i j}, u_{i j} \in \Lambda$ and $\overline{t_{i j}}, \overline{u_{i j}}(+, \cdot)$-prime in $\bar{\Lambda}$. Suppose that $\bar{t}=\bar{u}$. Since the $(+, \cdot)$-primes of $\bar{\Lambda}$ are algebraically independent, the above representations of $t$ and $u$ must coincide up to a permutation of summands in $\sum_{i}$ and permutations of factors in the corresponding $\prod_{j}$ 's.

\section{ON EXPONENTIAL FIELDS OF FUNCTIONS}

2.0. Preliminary remarks. In this section we consider the exponential field ${ }^{6}$ generated by the meromorphic functions (on any connected complex-analytic manifold). Such fields admit analogues of the Hiromi-Ozawa lemma and related results; see $\S 1.3$.

Example. Let $U \subset \mathbf{C}$ and $f_{j} \in \mathscr{M}(U)$ for $1 \leq j \leq n$. Let $f_{k}-f_{l} \notin \mathscr{H}(U)$ for $k \neq 1$. Then functions $\left\{e^{f_{j}}\right\}_{j=1}^{n}$ are linearly independent over $\mathscr{M}(U)$.

Proof. Suppose the contrary and take the minimal $n$ for which the statement fails. Let $g_{j} \in \mathscr{M}(U)-\{0\}$ for $1 \leq j \leq k$ and $\sum_{j=1}^{n} g_{j} e^{f_{j}}=0$. Then $\sum_{j=1}^{n-1} p_{j} e^{q_{j}}+1=0$, where $p_{j}=g_{j} / g_{n}$ and $q_{j}=f_{j}-f_{n}$ for $1 \leq j \leq n-1$. Note that $q_{k}-q_{l}=f_{k}-f_{l} \notin \mathscr{H}(U)$. By differentiation $\sum_{j=1}^{n-1}\left(p_{j} q_{j}^{\prime}+p_{j}^{\prime}\right) e^{q_{j}}=0$, whence $p_{j} q_{j}^{\prime}+p_{j}^{\prime}=0$ for $1 \leq j \leq n-1$ (by the minimality of $n$ ). The latter identity means that $\left(p_{j} e^{q_{j}}\right)^{\prime}=0$ and $p_{j} e^{q_{j}}=$ const for $1 \leq j \leq n-1$. However $e^{q_{j}}=e^{f_{j}-f_{n}}$ is not meromorphic (it has an essential singularity since $f_{j}-f_{n}$ has a pole) whereas $p_{j}$ is meromorphic. Hence $p_{j} e^{q_{j}}=$ const implies $p_{j}=0$. Thus $g_{j}=0$ for $1 \leq j \leq n-1$ and then $g_{n}=0$ too.

Before we turn to the statements of results we want to make some definitions. Note that the ring of entire functions $\mathscr{H}\left(\mathbf{C}^{n}\right)$ is not a field and its field of quotients $\mathscr{M}\left(\mathbf{C}^{n}\right)$ consists of some analytic functions defined not on the whole of $\mathbf{C}^{n}$ but only "almost everywhere". Similarly, $\mathscr{M}\left(\mathbf{C}^{n}\right)$ is not closed under exponentiation exp : $f \mapsto e^{f}$, and the analytic functions obtained from meromorphic ones by iterated field operations and exp are again defined only almost everywhere, i.e., outside sets which can be regarded as small. We need a formal definition of a class of analytic functions defined almost everywhere which will be closed under composition.

Definition. A closed proper subset $K$ of a connected manifold $U$ will be called exceptional if $W-K$ is connected for every connected open $W \subset U$.

It is well known that analytic subsets of complex-analytic manifolds ${ }^{7}$ are exceptional (see, e.g., [33]). It is less well known that for $n>1$ a closed subset of $\mathbf{R}^{n}$ is exceptional if and only if it has topological dimension $\leq n-2$; see $\S \S 4,5$ of [1]. (The "if" part is relatively easy, see Theorem IV:4 of [23].) In the case of $n=2$ the dim $\leq n-2$ result mentioned means that a closed subset of $\mathbf{C}$ is exceptional if and only if it is totally disconnected.

\footnotetext{
${ }^{6} \mathrm{An}$ exponential field is a pair $(F, \exp )$, where $F$ is a field and exp is a homomorphism from the additive group of $F$ into the multiplicative group $F-\{0\}$. For fields of complex-valued functions $\exp : f \mapsto e^{f}$.

${ }^{7} \mathrm{~A}$ set $X \subset W$ is called an analytic subset of $W$ if for every $\xi \in W$ there exists a neighborhood $U$ of $\xi$ in $W$ and $f_{1}, \ldots, f_{k} \in \mathscr{H}(U)-\{0\}$ such that $X \cap U=\bigcap_{j=1}^{k} f_{j}^{-1}(0)$.
} 
Remarks. (i) If $A$ and $B$ both are exceptional then $A \cup B$ is exceptional. If $K$ is exceptional in $U$ and $W$ is a connected open subset of $U$ then $K \cap W$ is exceptional in $W$. Obvious.

(ii) Let $X$ be a connected manifold, $\operatorname{dim}(X)>1$, and $K \subset X$. If for every $x \in X$ there exists a neighborhood $U$ of $x$ such that $K \cap U$ is exceptional in $U$ then $K$ is exceptional in $X$.

Trivially, $K$ is closed. Let $Y$ be an open connected subset of $X$. For each $x \in Y$ fix an open ball neighborhood $U_{x}$ of $x$ in $Y$ so small that $K \cap U_{x}$ is exceptional in $U_{x}$. Let $p, q \in Y-K$. Since $Y$ is connected, there exist $x_{0}, \ldots, x_{n} \in Y$ such that $p \in U_{x_{0}}, q \in U_{x_{n}}$, and $U_{x_{j-1}} \cap U_{x_{j}} \neq \varnothing$ for $1 \leq j \leq n$. Since all $K \cap U_{x}$ 's are nowhere dense, $\left(U_{x_{j-1}}-K\right) \cap\left(U_{x_{j}}-K\right) \neq \varnothing$ for $1 \leq j \leq n$, and since each $U_{x}-K$ is connected, $p$ and $q$ belong to the same connected component of $Y-K$.

(iii) A subset $K$ of a connected topological manifold $X$ with $\operatorname{dim}(X)>1$ is exceptional if and only if $\operatorname{dim}(K) \leq \operatorname{dim}(X)-2$. This follows immediately from (ii) and the case of $X=\mathbf{R}^{n}$.

(iv) If $K$ is an exceptional subset of a connected manifold $X$ with $\operatorname{dim}(X)>$ 1 and $p, q \in X-K$ then the set of paths in $X-K$ from $p$ to $q$ is dense (in the compact-open topology) in the set of all paths in $X$ from $p$ to $q$.

Let $\lambda:[0,1] \rightarrow X, \lambda(0)=p, \lambda(1)=q$. Let us take a neighborhood of $\lambda$ in the space of paths in $X$ and find a path $\alpha$ in $X-K$ from $p$ to $q$ belonging to that neighborhood of $\lambda$. Let $\left\{U_{j}\right\}_{j=1}^{n}$ be open balls in $X$, let $t_{0}=0<t_{1}<\cdots<t_{n-1}<t_{n}=1$, and $\lambda\left(\left[t_{j-1}, t_{j}\right]\right) \subset U_{j}$ for $1 \leq j \leq n$. Then $U_{j} \cap U_{j+1} \neq \varnothing$ for $1 \leq j<n$. Since $K$ is nowhere dense, for each $j$, $1 \leq j<n$, there exists a $u_{j} \in\left(U_{j} \cap U_{j+1}\right)-K$. Put also $u_{0}=p, u_{n}=q$. Since each $U_{j}-K$ is connected, it is also arcwise connected. Hence there exist paths $\alpha_{j}:\left[t_{j-1}, t_{j}\right] \rightarrow U_{j}-K$ with $\alpha_{j}\left(t_{j-1}\right)=u_{j-1}$ and $\alpha_{j}\left(t_{j}\right)=u_{j}$. Together $\left\{\alpha_{j}\right\}_{j=1}^{n}$ form a path from $p$ to $q$ in $X-K$ which belongs to the neighborhood of $\lambda$ chosen.

(v) If $f: U \rightarrow W$ is a holomorphic mapping of connected complex-analytic manifolds, $f(U)=W$, and $K \subset W$ is exceptional then $f^{-1}(K)$ is an exceptional subset of $U$.

Note first that if $U=V \times W$ for some complex-analytic manifold $V$ and $f$ is the projection of the product $U$ onto the second factor then the result is trivial. Consider the general case. Since $f$ is onto, the set $C$ of critical points of $f$ (i.e., of those points $u \in U$ at which the differential $T_{u} f: T_{u} U \rightarrow T_{u} W$ has rank $<\operatorname{dim}(W))$ is an analytic subset of $U$ and hence is exceptional in $U$. Thus it suffices to show that $(U-C) \cap f^{-1}(K)$ is exceptional in $U-C$. Upon replacing $U$ by $U-C$ and $W$ by $f(U-C)$ one can assume that $f$ has no critical points. Let $V$ be an open connected subset of $U$ and $p, q \in$ $V-f^{-1}(K)$. Let $\lambda$ be a path in $V$ from $p$ to $q$. For each $v \in \lambda([0,1])$ let $D_{v} \subset V$ be a polydisk neighborhood of $v$ such that the restriction of $f$ to $D_{v}$ is isomorphic to a projection $D_{v}^{\prime} \times D_{v}^{\prime \prime} \rightarrow D_{v}^{\prime}$ for some decomposition $D_{v}=$ $D_{v}^{\prime} \times D_{v}^{\prime \prime}$ of $D_{v}$ into a product of polydisks. Since $\lambda([0,1])$ is compact, one can cover it with finitely many such polydisks. Thus one can assume that there are $n$ such polydisks, $\left\{D_{j}\right\}_{j=1}^{n}$, and $\lambda([0,1]) \subset \bigcup_{j=1}^{n} D_{j}$ and $D_{j} \cap D_{j+1} \neq \varnothing$ for $1 \leq j<n$. Let $u_{j} \in\left(D_{j} \cap D_{j+1}\right)-f^{-1}(K)$ for $1 \leq j<n$. Such $u_{j}$ 's exist because $f^{-1}$ (a nowhere dense set) is nowhere dense for every open mapping 
$f$. Denote also $u_{0}=p, u_{n}=q$. Since the result is trivial for $f$ a coordinate projection, for each $j, 1 \leq j \leq n$, there exists a path $\alpha_{j}:[j-1, j] \rightarrow$ $D_{j}-f^{-1}(K)$ with $\alpha_{j}(j-1)=u_{j-1}, \alpha_{j}(j)=u_{j}$. Then $\left\{\alpha_{j}\right\}_{j}$ together form a path $[0, n] \rightarrow V-f^{-1}(K)$ from $p$ to $q$.

Let $K$ be an exceptional subset of a connected complex-analytic manifold $U$ (for example, consider $U$ a polydisk), and $f \in \mathscr{H}(U-K)$. One can say that $K$ is a set of singularities of $f$, but some of these singularities may be removable.

Proposition. Let $U$ be a connected complex-analytic manifold, $K$ be an exceptional subset of $U$, and $f \in \mathscr{H}(U-K)$. Let $x \in K$. If there exists a path $\lambda$ in $U$ such that $\lambda([0,1)) \subset U-K, \lambda(1)=x$, and $f$ can be continued analytically along $\lambda$ then $f \in \mathscr{H}\left(U-K^{\prime}\right)$ for some closed set $K^{\prime} \subset K, x \notin K^{\prime}$.

It is important that $K$ is exceptional. For example, $\log z \in \mathscr{H}(\mathbf{C}-(-\infty, 0])$ can be continued analytically along any path not passing through $0 \in \mathbf{C}$, but cannot be extended to a holomorphic function on a larger subset of $\mathbf{C}$.

Proof. Recall that an analytic continuation of $f$ along a path $\lambda:[0,1] \rightarrow U$ is a family $\left\{\left(t_{j}, U_{j}, f_{j}\right)\right\}_{j=0}^{n}$, where $t_{0}=0<t_{1}<\cdots<t_{n-1}<t_{n}=1$, each $U_{j}$ is a connected open neighborhood of $\lambda\left(t_{j}\right)$ and $f_{j} \in \mathscr{H}\left(U_{j}\right)$, such that $U_{j-1} \cap U_{j} \neq \varnothing$ for $1 \leq j \leq n, f_{0}=\left.f\right|_{U_{0}}$ and $f_{j}$ coincides with $f_{j-1}$ on $U_{j-1} \cap U_{j}$ for $1 \leq j \leq n$.

Let $\lambda([0,1)) \subset U-K$ and $\lambda(1) \in K$. Show that $f_{j}$ coincides with $f$ on $U_{j}-K$ (the latter set is nonempty because $K$ is nowhere dense) for all $j$. By the definition, this holds for $j=0$. Suppose that $f_{j}$ coincides with $f$ on $U_{j}-K$ and consider $f_{j+1}$. Since $K$ is nowhere dense, $\left(U_{j} \cap U_{j+1}\right)-K \neq \varnothing$, and $f_{j+1}$ coincides with $f_{j}$ and hence with $f$ on $\left(U_{j} \cap U_{j+1}\right)-K$. Since $U_{j+1}-K$ is connected and $f_{j+1}$ coincides with $f$ on an open subset of it, $f_{j+1}$ coincides with $f$ on the whole of $U_{j+1}-K$. Thus $f_{n}$ coincides with $f$ on $U_{n}-K$, and

$$
\tilde{f}(u)= \begin{cases}f(u) & \text { if } u \in U-K, \\ f_{n}(u) & \text { if } u \in U_{n}\end{cases}
$$

is a holomorphic function on $(U-K) \cup U_{n}$.

Thus one can consider the set of nonremovable singularities of $f$; denote by $\mathbf{S}_{f}$ the set of points of $K$ to which $f$ cannot be analytically continued. Then $\mathbf{S}_{f}$ is an exceptional set too. If $K$ is an analytic subset of $U$ and $f \in \mathscr{H}(U-K)$ then $\mathbf{S}_{f}$ is an analytic subset of $U$ (then $\mathbf{S}_{f}$ is $K$ minus some of the components of $K)$. This fact ${ }^{8}$ provokes us to state the following.

Conjecture. For any holomorphic mapping $h$ of a connected complex-analytic manifold $W$ into $U$ either $h(W) \subset \mathbf{S}_{f}$ or $h^{-1}\left(\mathbf{S}_{f}\right)$ is exceptional in $W$.

This conjecture is an analogue of the fact that for every holomorphic mapping $h: W \rightarrow U$ of connected complex-analytic manifolds and any analytic subset $V \subset U$ either $h(W) \subset V$ or $h^{-1}(V)$ is an analytic subset of $W$. A particular case equivalent to the whole of the above conjecture is: if $g$ is a holomorphic

\footnotetext{
${ }^{8}$ We do not need this in the sequel. A proof would be a standard exercise in several complex variables theory.
} 
mapping on the unit disk $D$ into $U$ then either $g(D) \subset \mathbf{S}_{f}$ or $g(D) \cap \mathbf{S}_{f}$ is totally disconnected.

Let $\mathbf{S}$ be a proper closed subset of $U$ such that for every holomorphic mapping $g: D \rightarrow U$ either $g(D) \subset \mathbf{S}$ or $g(D) \cap \mathbf{S}$ is totally disconnected. (Then $\mathbf{S}$ is an exceptional subset of $U$.) The question of whether such $\mathbf{S}$ is actually of the form $\mathbf{S}_{f}$ is an analogue of the classical question of whether a given analytic subset is a variety. We suspect that each $\mathbf{S}$ satisfying the above conditions is of the form $\mathbf{S}_{f}$ (whereas not every analytic subset is a variety).

Definition. For a connected complex-analytic manifold $U$ put

$$
\mathscr{E}(U)=\bigcup\{\mathscr{H}(U-K) \mid K \text { an exceptional subset of } U\} \text {. }
$$

Functions in $\mathscr{E}(U)$ will be called almost everywhere (a.e.) holomorphic on $U$.

Then $\mathscr{E}$ is a sheaf of fields and every meromorphic function is holomorphic almost everywhere. Each $\mathscr{E}(U)$ obviously admits left composition with meromorphic functions: if $K$ is an exceptional subset of $U, f: U-K \rightarrow W$ is a holomorphic mapping, $g \in \mathscr{M}(W)$, and $f(U-K)$ is not contained in the polar set of $g$ then $g \circ f \in \mathscr{E}(U)$. In particular, each $\mathscr{E}(U)$ is closed under exponentiation. Some examples of functions in $\mathscr{E}(\mathbf{C})$ are the following:

$$
1 / z, \quad e^{1 / \sin (z)}, \quad \tan (\tan z), \quad \sum_{n=1}^{\infty} \frac{1}{n !\left(z-t_{n}\right)^{n}},
$$

where $\left\{t_{n}\right\}_{n}$ is a dense subset of the Cantor set, and in $\mathscr{E}\left(\mathbf{C}^{2}\right)$ :

$$
\sin \left(e^{\tan (\tan z)}+\Gamma\left(\frac{z+w}{z-w}\right)\right), \quad e^{\sqrt{\Gamma(\tan z+w)}}+e^{-\sqrt{\Gamma(\tan z+w)}} .
$$

The following functions, though defined on $\mathbf{C}-\{$ a very small set $\}$, do not belong to $\mathscr{E}(\mathbf{C})$ :

$$
\sqrt{z(z-2)}, \quad \sqrt[3]{z(z-1)(z-2)} \in \mathscr{H}(\mathbf{C}-[0,2]) .
$$

The reason is that they cannot be extended to holomorphic functions on subsets of the disk $|z|<1$ containing loops around 0 , while every exceptional $K \subset \mathbf{C}$ is totally disconnected and hence (the open unit disk) $-K$ contains loops around every point of $K$.

\subsection{Lemmata.}

Remark. Let $U$ be a real-analytic submanifold of $\mathbf{R}^{m}$. Then for every $u \in U$ there exists a neighborhood $W$ of $u$ in $\mathbf{R}^{m}$ such that the function

$$
p: x \mapsto \text { (the point of } U \text { nearest to } x \text { in the Euclidean distance) }
$$

is correctly defined and real-analytic on $W$.

Lemma 1. Let $V$ be a real-analytic manifold. Let $t_{1}, \ldots, t_{k} \in S^{1}$, where $S^{1}$ is the unit circle $|z|=1$ in $\mathbf{C}$, and $v_{1}, \ldots, v_{k} \in V$. The set of real-analytic mappings $\lambda: S^{1} \rightarrow V$ satisfying $\lambda\left(t_{j}\right)=v_{j}, 1 \leq j \leq k$, is dense for every $l=1,2, \ldots, \infty$ in the set of all $C^{l}$ (i.e., $l$ times differentiable) mappings $h: S^{1} \rightarrow V$ satisfying $h\left(t_{j}\right)=v_{j}, 1 \leq j \leq k$, in the usual $C^{l}$-mappings topology.

This implies in particular that every element of $\pi_{1}(V, a)$ can be represented by a real-analytic mapping $\left(S^{1}, 1\right) \rightarrow(V, a)$, since every two sufficiently close 
mappings $\left(S^{1}, 1\right) \rightarrow(V, a)$ are homotopic. The lemma can be easily generalized to mappings $X \rightarrow V$ for arbitrary real-analytic manifolds $X$, but we do not need this generalization in this paper.

Proof. By [31] one can assume that $V$ is a submanifold of $\mathbf{R}^{m}$ for some $m$. Let $h: S^{1} \rightarrow V$ and $h\left(t_{j}\right)=v_{j}$ for all $j$. Denote $A=h\left(S^{1}\right)$. Since $A$ is compact there is a neighborhood $G$ of $A$ in $\mathbf{R}^{m}$ such that the function $p: x \mapsto$ (the point of $V$ nearest to $x$ ) is correctly defined and real-analytic on $G$. Approximate $h: S^{1} \rightarrow \mathbf{R}^{m}$ with a sequence of real-analytic functions $g_{n}: S^{1} \rightarrow \mathbf{R}^{m}$ satisfying $g_{n}\left(t_{j}\right)=v_{j}$ for all $j$ and converging to $h$ in the $C^{l}$ mappings topology. Then for a sufficiently large $n$ the function $p \circ g_{n}: S^{1} \rightarrow V$ will be as close to $h$ (in the topology of the space of $C^{l}$-loops in $V$ ) as required.

Lemma 2. Let $V$ be a complex-analytic manifold and $h: S^{1} \rightarrow V$ be a realanalytic mapping, $S^{1}=\{z \in \mathbf{C}|| z \mid=1\}$. Then there exists a unique complexanalytic extension $g:\{z \in \mathbf{C}|1-\varepsilon<| z \mid<1+\varepsilon\} \rightarrow V$ of $h$ for sufficiently small $\varepsilon>0$.

Proof. Clearly, for each $z \in S^{1}$ there exists an $\varepsilon_{z}>0$ such that $h$ can be analytically continued to a mapping of the disk $|w-z|<\varepsilon_{z}$ into $V$. Since $S^{1}$ is compact there is an $\varepsilon>0$ so small that for every $u \in S^{1}$ the disk $|w-u|<\varepsilon$ is contained in one of the disks $|w-z|<\varepsilon_{z}$. Then one can continue $h$ analytically to every point of the annulus $1-\varepsilon<|w|<1+\varepsilon$ starting at $z=w /|w|$ and going along the radius $\{t z\}_{1-\varepsilon<t<1+\varepsilon}$.

2.2. The exponential field generated by meromorphic functions: formulations of results. Let $U$ be a connected complex-analytic manifold and $\mathscr{F}(U)$ be the minimal subfield of $\mathscr{E}(U)$ containing $\mathscr{M}(U)$ and closed under exponentiation.

Theorem 1. Let $f_{j}, g_{k} \in \mathscr{F}(U)$ and $p_{j}, q_{k} \in \mathscr{M}(U)-\{0\}$ for $1 \leq j \leq m$, $1 \leq k \leq n$. If

$$
\frac{\sum_{j=1}^{m} p_{j} e^{f_{j}}}{\sum_{k=1}^{n} q_{k} e^{g_{k}}}=h \in \mathscr{M}(U),
$$

and $f_{j}-f_{j^{\prime}} \notin \mathscr{H}(U)$ for $j \neq j^{\prime}$ and $g_{k}-g_{k^{\prime}} \notin \mathscr{H}(U)$ for $k \neq k^{\prime}$, then $m=n$ and there exists a permutation $\sigma$ of $\{1, \ldots, m\}$ such that $f_{j}-g_{\sigma(j)} \in \mathscr{H}(U)$ and $h=\left(p_{j} / q_{\sigma(j)}\right) \cdot e^{f_{j}-g_{\sigma(j)}}$ for $1 \leq j \leq m$.

This theorem admits a strengthening which is most easily formulated for $U$ being one-dimensional.

Proposition 2. Let $U$ be 1-dimensional. Let $f_{j}, g_{k} \in \mathscr{F}(U)$ and $p_{j}, q_{k} \in$ $\mathscr{M}(U)-\{0\}$ for $1 \leq j \leq m, 1 \leq k \leq n$. Let $f_{j}-f_{j^{\prime}} \notin \mathscr{H}(U)$ for $j \neq j^{\prime}$ and $g_{k}-g_{k^{\prime}} \notin \mathscr{H}(U)$ for $k \neq k^{\prime}$. If the set of zeros and singularities of

$$
h=\frac{\sum_{j=1}^{m} p_{j} e^{f_{j}}}{\sum_{k=1}^{n} q_{k} e^{g_{k}}}
$$

is discrete then $m=n$ and there exists a permutation $\sigma$ of $\{1, \ldots, m\}$ such that $h=\left(p_{j} / q_{\sigma(j)}\right) \cdot e^{f_{j}-g_{\sigma(j)}}$ for $1 \leq j \leq m$.

For multidimensional $U$ the analogue of "all singularities are isolated" is "the set of singularities (if nonempty) is an analytic subset". 
Theorem 3. Let $f_{j}, g_{k} \in \mathscr{F}(U)$ and $p_{j}, q_{k} \in \mathscr{M}(U)-\{0\}$ for $1 \leq j \leq m$, $1 \leq k \leq n$. Let $f_{j}-f_{j^{\prime}} \notin \mathscr{H}(U)$ for $j \neq j^{\prime}$ and $g_{k}-g_{k^{\prime}} \notin \mathscr{H}(U)$ for $k \neq k^{\prime}$. If the set $\{$ the zeros of $h\} \cup\{$ the singularities of $h\}$ is contained in a codimension 1 analytic subset of $U$, where

$$
h=\frac{\sum_{j=1}^{m} p_{j} e^{f_{j}}}{\sum_{k=1}^{n} q_{k} e^{g_{k}}}
$$

then $m=n$ and there exists a permutation $\sigma$ of $\{1, \ldots, m\}$ such that $h=$ $\left(p_{j} / q_{\sigma(j)}\right) \cdot e^{f_{j}-g_{\sigma(j)}}$ for $1 \leq j \leq m$.

These results allow one to construct for the exponential field $\mathscr{F}(U)$ an analogue of the van den Dries normal form for free exponential rings; see [8]. It is known how to describe in a similar way the algebraic structure of the "free exponential field" obtained from an exponential field and a number of indeterminates; see, e.g., A. Macintyre's notes on exponentiation. Using Theorem 1 one can show immediately that the minimal exponential subfield of $\mathscr{E}\left(\mathbf{C}^{m}\right)$ containing all rational functions with coefficients in $\mathbf{C}$ is indeed the free exponential field over $\mathbf{C}$ generated by indeterminates $\left\{z_{j}\right\}_{j=1}^{m}$ : consider the $m$ th power of the Riemann sphere instead of $\mathbf{C}^{m}$, the meromorphic functions on it are exactly the rational functions on $\mathbf{C}^{m}$.

Recall that in analogy with semigroup rings $R[S]$ ( $R$ a ring, $S$ a semigroup) one can consider group fields $F(G)$ for fields $F$ and torsion-free Abelian groups $G$ : put $F(G)=$ the field of quotients of $F[G]$. Then $F$ is naturally embedded into $F(G)$, and $F(G)$ is a linear space over $F$.

Now turn to $\mathscr{F}(U)$. Put $F_{0}=\mathscr{M}(U)$ and let $S_{0}$ be an additive subgroup of $\mathscr{M}(U)$ complementing $\mathscr{H}(U)$, i.e., $S_{0} \cap \mathscr{H}(U)=\{0\}$ and $S_{0}+\mathscr{H}(U)=\mathscr{M}(U)$. For $n \geq 1$ put $F_{n}=$ the minimal subfield of $\mathscr{E}(U)$ containing $F_{n-1} \cup\left\{e^{f} \mid f \in\right.$ $\left.F_{n-1}\right\}$ and let $S_{n}$ be any additive subgroup of $F_{n}$ complementing $F_{n-1}$ in $F_{n}$ (i.e., $\left.F_{n}=S_{n} \oplus F_{n-1}\right)$. Clearly $\mathscr{F}(U)=\bigcup_{n} F_{n}$.

Theorem 4. In the above notation, each $F_{n}$ is isomorphic to $F_{n-1}\left(S_{n-1}\right)$, i.e., the set $\exp \left(S_{n-1}\right)$ is linearly independent over $F_{n-1}$.

It is not an accident that Theorems 1 and 3 resemble the Hiromi-Ozawa lemma; they are actually its easy corollaries. We would prefer to prove those theorems in a more differentially algebraic way: we feel that a statement holding for an arbitrary manifold should admit a proof not based on Nevanlinna theory. But at present we do not don't have a purely differentially algebraic proof of the results of this section.

Remark 5. Let $\mathscr{R} \subset \mathscr{H}(U)$ be an exponential ring such that $f / g \in \mathscr{R}$ whenever $f, g \in \mathscr{R}$ and $f / g \in \mathscr{H}(U)$. Then, for every $f \in \mathscr{H}(U)$ if $f$ belongs to the exponential field generated by $\mathscr{R}$ then $f \in \mathscr{R}$.

Proof. Denote $F_{0}=$ the field of quotients of $\mathscr{R}$, and $F_{n+1}=$ the field generated by $F_{n} \cup\left\{e^{h} \mid h \in F_{n}\right\}$. Let $f \in \mathscr{H}(U) \cap$ the exponential field generated by $\mathscr{R}\}$, and proceed by induction on $k=\min \left\{n \mid f \in F_{n}\right\}$. The case $k=0$ is trivial. Let $k>0$. Then (using the induction assumption) one can present $f$ in the form

$$
f=\frac{\sum_{j=1}^{m} p_{j} e^{g_{j}}}{\sum_{l=1}^{n} q_{l} e^{h_{l}}},
$$


for some $p_{j}, q_{l} \in \mathscr{R}-\{0\}, g_{j}, h_{l} \in F_{k-1}$ with $g_{j}-g_{j^{\prime}} \notin \mathscr{H}(U)$ for $j \neq j^{\prime}$ and $h_{l}-h_{l^{\prime}} \notin \mathscr{H}(U)$ for $l \neq l^{\prime}$. By Theorem $1, m=n$ and there exists a permutation $\sigma$ of $\{1, \ldots, m\}$ such that $g_{1}-h_{\sigma(1)} \in \mathscr{H}(U), f=\left(p_{1} / q_{\sigma(1)}\right)$. $e^{g_{1}-h_{\sigma(1)}}$. Then $g_{1}-h_{\sigma(1)} \in \mathscr{R}$ (by the induction assumption), whence $f \in \mathscr{R}$.

Below we provide a nontrivial example of $\mathscr{R}$ with the properties required in Remark 5 (a trivial example would be $\mathscr{R}=\mathscr{H}(U)$ ) but we want to make a comment before. It was proved by Ritt that if $a_{j}, \lambda_{j}, b_{k}, \mu_{k} \in \mathbf{C}$ and

$$
h=\frac{\sum_{j} a_{j} e^{\lambda_{j} z}}{\sum_{k} b_{k} e^{\mu_{k} z}}
$$

is entire then $h=\sum_{l} c_{l} e^{\nu_{l} z}$ for some $c_{l}, \nu_{l} \in \mathbf{C}$. We asked L. A. Rubel whether "if $f_{j}, g_{k} \in \mathscr{H}(\mathbf{C})$ and

$$
H=\frac{\sum_{j} e^{f_{j}(z)}}{\sum_{k} e^{g_{k}(z)}}
$$

is entire then $H=\sum_{l} e^{h_{l}(z)}$ for some $h_{l} \in \mathscr{H}(\mathbf{C})$ " and he provided the answer: no,

$$
\frac{\sin \left(\pi z^{2}\right)}{\sin (\pi z)} \text { and } \frac{\sin (\sin z)}{\sin z}
$$

are entire, but are not sums of exponentials. To prove Rubel's remark one has to use the Hiromi-Ozawa lemma (see §1.3).

Example 6. The exponential field generated by $\left\{e^{f} \mid f \in \mathscr{H}\left(\mathbf{C}^{n}\right)\right\}$ contains no meromorphic functions of order $<1$ and no meromorphic functions of order 1 with the minimal type. ${ }^{9}$

Thus $\mathscr{R}=\mathscr{H}\left(\mathbf{C}^{n}\right) \cap$ (the exponential field generated by $\left\{e^{f} \mid f\right.$ is entire $\left.\}\right)$ is an example for Remark 5 above.

Proof. Denote $F_{0}=$ the field generated by $\left\{e^{f} \mid f\right.$ is entire $\}$, and $F_{n+1}=$ the field generated by $F_{n} \cup\left\{e^{h} \mid h \in F_{n}\right\}$. Let $f \in\left(\bigcup_{n \in \mathrm{N}} F_{n}\right) \cap \mathscr{M}\left(\mathbf{C}^{n}\right)$ and $k=$ $\min \left\{n \mid f \in F_{n}\right\}$. To show that $f$ cannot have order $<1$ or order 1 with the minimal type proceed by induction on $k$. Suppose the contrary: $T(r, f)=$ $o\left(T\left(r, e^{g}\right)\right)$ for all nonconstant entire $g$.

Case $k=0$. Then $f=\left(\sum_{j} e^{g_{j}}\right) /\left(\sum_{l} e^{h_{l}}\right)$ for some entire $g_{j}, h_{l}$ with $g_{j}-g_{j^{\prime}} \neq$ const for $j \neq j^{\prime}$ and $h_{l}-h_{l^{\prime}} \neq$ const for $l \neq l^{\prime}$. Since $f$ is Nevanlinna-small compared to all $e^{g_{j}-g_{j^{\prime}}\left(j \neq j^{\prime}\right)}$ and $e^{h_{l}-h_{l^{\prime}}}\left(l \neq l^{\prime}\right)$, by the Hiromi-Ozawa lemma (§1.3) functions $\left\{e^{g_{j}}\right\}_{j} \cup\left\{f \cdot e^{h_{l}}\right\}_{l}$ are linearly dependent over $\mathbf{C}$. Thus $f$ can be presented by a fraction with less total number of summands in numerator and denominator. Iterating this reasoning one arrives at $f=e^{g}, g \in \mathscr{H}\left(\mathbf{C}^{n}\right)-\mathbf{C}$, contradicting the assumption that $f$ is Nevanlinna-small.

Case $k>0$. Then (by induction on $k$ )

$$
f=\frac{\sum_{j=1}^{J} p_{j} e^{g_{j}}}{\sum_{l=1}^{L} q_{l} e^{h_{l}}},
$$

\footnotetext{
${ }^{9}$ For and $f$ to have order $<1$ or order 1 with the minimal type is equivalent to $T(r, f)=$ $o\left(T\left(r, e^{g}\right)\right)$ for all nonconstant entire $g$.
} 
for some $g_{j}, h_{l} \in F_{k-1}$ and $p_{j}, q_{l} \in$ (the ring generated by $\left\{e^{t} \mid t\right.$ is entire $\}$ ) $\{0\}$, with $g_{j}-g_{j^{\prime}} \notin \mathscr{H}\left(\mathbf{C}^{n}\right)$ for $j \neq j^{\prime}$ and $h_{l}-h_{l^{\prime}} \notin \mathscr{H}\left(\mathbf{C}^{n}\right)$ for $l \neq l^{\prime}$. Then by Theorem 3 there are $\alpha, \beta$ such that $f=\left(p_{\alpha} e^{g_{\alpha}}\right) /\left(q_{\beta} e^{h_{\beta}}\right)=\left(p_{\alpha} / q_{\beta}\right) \cdot e^{g_{\alpha}-h_{\beta}}$. If $g_{\alpha}-h_{\beta} \notin \mathscr{H}\left(\mathbf{C}^{n}\right)$ then $\left(p_{\alpha} / q_{\beta}\right) \cdot e^{g_{\alpha}-h_{\beta}}$ has an essential singularity whereas $f$ is meromorphic. Thus $g_{\alpha}-h_{\beta} \in \mathscr{H}\left(\mathbf{C}^{n}\right)$ and $f=\left(\sum_{\mu} e^{s_{\mu}}\right) /\left(\sum_{\nu} e^{t_{\nu}}\right)$ for some entire $s_{\mu}, t_{\nu}$. This reduces the situation to the case $k=0$.

2.3. The exponential field generated by meromorphic functions: proofs. In this subsection we prove Theorems 1,3 and 4 of $\S 2.2$. First we shall prove Proposition 2 of $\S 2.2$ (which is a particular case of Theorem 3 of $\S 2.2$ ), and then we shall use it as a lemma for the more general results. We begin with the case of $U$ being the unit disk $D=\{z \in \mathbf{C}|| z \mid<1\}$. Denote by $\mu$ the universal covering $D \rightarrow D-\{0\}$ defined by the formula

$$
z=\mu(u)=e^{(u+1) /(u-1)} .
$$

Then $z \rightarrow 0$ if $u \rightarrow 1$ nontangentially; in particular,

$$
\max _{r-1<\theta<1-r}\left|\mu\left(r e^{i \theta}\right)\right| \rightarrow 0, \quad \text { as } r \rightarrow 1 .
$$

Lemma 1. Let $f \in \mathscr{H}(D), f(0) \neq 0$, and $k \in \mathbf{N}$. Then

$$
e^{2 k /(1-r)} \cdot(1-r)^{1.5}=O\left(T\left(r,\left(e^{f(z) / z^{k}}\right) \circ \mu\right)\right),
$$

as $r \rightarrow 1$, where $T(r, \cdot)$ is the Nevanlinna characteristic.

Proof. In analogy with $\log ^{+}|\cdot|$, denote $\operatorname{re}^{+}(w)=\max (0$, re $w)$ for $w \in \mathbf{C}$ and $(x)^{+}=\max (0, x)$ for $x \in \mathbf{R}$. Recall that $\log ^{+}\left|e^{w}\right|=\operatorname{re}^{+}(w)$ for all $w \in \mathbf{C}$. So,

$$
T\left(r,\left(e^{f(z) / z^{k}}\right) \circ \mu\right)=\frac{1}{2 \pi} \int_{-\pi}^{\pi} \mathrm{re}^{+}\left((f \circ \mu)\left(r e^{i \theta}\right) \cdot e^{k \cdot\left(1+r e^{i \theta}\right) /\left(1-r e^{i \theta}\right)}\right) d \theta .
$$

We are going to prove that

$$
\int_{-(1-r)^{1.5}}^{(1-r)^{1.5}} \mathrm{re}^{+}\left((f \circ \mu)\left(r e^{i \theta}\right) \cdot e^{k \cdot\left(1+r e^{i \theta}\right) /\left(1-r e^{i \theta}\right)}\right) d \theta \geq \mathrm{const} \cdot(1-r)^{1.5} \cdot e^{2 k /(1-r)}
$$

as $r \rightarrow 1$. Note first that

$$
\left|\frac{1+r e^{i \theta}}{1-r e^{i \theta}}\right|<\frac{2}{1-r}
$$

whence

$$
\left|e^{k \cdot\left(1+r e^{i \theta}\right) /\left(1-r e^{i \theta}\right)}\right|<e^{2 k /(1-r)}
$$

and

$$
\int_{-(1-r)^{1.5}}^{(1-r)^{1.5}}\left|e^{k \cdot\left(1+r e^{i \theta}\right) /\left(1-r e^{i \theta}\right)}\right| d \theta<2(1-r)^{1.5} \cdot e^{2 k /(1-r)} .
$$

Consequently, for any $g \in \mathscr{H}(D)$

$$
\int_{-(1-r)^{1.5}}^{(1-r)^{1.5}}\left|(g \circ \mu)\left(r e^{i \theta}\right) \cdot e^{k\left(1+r e^{i \theta}\right) /\left(1-r e^{i \theta}\right)}\right| d \theta<2 G(r) \cdot(1-r)^{1.5} \cdot e^{2 k /(1-r)}
$$

where $G(r)$ is the maximum of $\left|(g \circ \mu)\left(r e^{i \theta}\right)\right|$ for $\theta \in\left[-(1-r)^{1.5},(1-r)^{1.5}\right]$. Put $a=f(0)$ and $g(z)=f(z)-a$. Then $G(r)$ is not more than

$$
\max _{r-1<\theta<1-r}\left|((f-a) \cdot \mu)\left(r e^{i \theta}\right)\right|,
$$


which tends to 0 as $r \rightarrow 1$, because $\mu(u)$ tends to 0 when $u \rightarrow 1$ nontangentially. Thus it suffices to estimate from below

$$
\int_{-(1-r)^{1.5}}^{(1-r)^{1.5}} \mathrm{re}^{+}\left(a \cdot e^{k \cdot\left(1+r e^{i \theta}\right) /\left(1-r e^{i \theta}\right)}\right) d \theta
$$

for arbitrary constant nonzero $a \in \mathbf{C}$. The latter means estimating from below

$$
\begin{aligned}
\int_{-(1-r)^{1.5}}^{(1-r)^{1.5}} e^{k \cdot\left(1-r^{2}\right) /\left(1-2 r \cos \theta+r^{2}\right)} & \\
& \cdot\left(b \cdot \cos \frac{2 k r \sin \theta}{1-2 r \cos \theta+r^{2}}+c \cdot \sin \frac{2 k r \sin \theta}{1-2 r \cos \theta+r^{2}}\right)^{+} d \theta,
\end{aligned}
$$

for arbitrary $b, c \in \mathbf{R}$ with $b^{2}+c^{2} \neq 0$, or

$$
\int_{-(1-r)^{1.5}}^{(1-r)^{1.5}} \exp \left(k \cdot \frac{1-r^{2}}{1-2 r \cos \theta+r^{2}}\right) \cdot\left(\cos \left(\frac{2 k r \sin \theta}{1-2 r \cos \theta+r^{2}}+p\right)\right)^{+} d \theta,
$$

for arbitrary $p \in \mathbf{R}$. Since $\cos \theta>1-\theta^{2} / 2$, for $\eta=(1-r)^{1.5}$

$$
\begin{aligned}
e^{k \cdot\left(1-r^{2}\right) /\left(1-2 r \cos \eta+r^{2}\right)} & >e^{k \cdot\left(1-r^{2}\right) /(1-r)^{2}\left(1+r-r^{2}\right)} \\
& =e^{k \cdot(1+r) /(1-r)\left(1+r-r^{2}\right)}>e^{-3} \cdot e^{2 k /(1-r)},
\end{aligned}
$$

and hence it will be enough for any fixed $p \in \mathbf{R}$ to show that

$$
\int_{-(1-r)^{1.5}}^{(1-r)^{1.5}}\left(\cos \left(\frac{2 k r \sin \theta}{1-2 r \cos \theta+r^{2}}+p\right)\right)^{+} d \theta>\text { const } \cdot(1-r)^{1.5},
$$

as $r \rightarrow 1$. Since $1>\cos \theta>1-\theta^{2} / 2$ and $\theta>\sin \theta>\theta-\theta^{3} / 6$ (for $\theta>0$ ),

$$
\max _{\theta \in\left[0,(1-r)^{1.5}\right]}\left|\frac{2 k r \theta}{(1-r)^{2}}-\frac{2 k r \sin \theta}{1-2 r \cos \theta+r^{2}}\right| \leq \text { const } \cdot(1-r)^{0.5},
$$

as $r \rightarrow 1$ and thus

$$
\max _{\theta \in\left[0,(1-r)^{1.5}\right]}\left|\cos \left(\frac{2 k r \theta}{(1-r)^{2}}+p\right)-\cos \left(\frac{2 k r \sin \theta}{1-2 r \cos \theta+r^{2}}+p\right)\right| \rightarrow 0,
$$

as $r \rightarrow 1$. Consequently, it suffices to check that

$$
\int_{A_{r}} \cos \left(\frac{2 k r \theta}{(1-r)^{2}}+p\right) d \theta>\text { const } \cdot(1-r)^{1.5},
$$

as $r \rightarrow 1$, where $A_{r}=\left\{\theta \in\left[0,(1-r)^{1.5}\right] \mid \cos \left(2 r \theta(1-r)^{-2}+p\right)>1 / 2\right\}$. The latter estimation follows immediately from the fact that

$$
g: \theta \mapsto 2 k r \theta(1-r)^{-2}+p
$$

is a linear mapping of $\left[0,(1-r)^{1.5}\right]$ onto the segment $\left[p, p+2 k r(1-r)^{-0.5}\right]$ whose length tends to $\infty$ as $r \rightarrow 1$.

The above estimation is exact: take $f(z)=1$. The universal covering of the unit disk without zero by the unit disk is given by an easy formula, and this fact helped very much in proving Lemma 1 . Another helpful circumstance was $f / z^{k} \in \mathscr{M}(D)$. It would be very interesting to find similar estimations for a universal covering $\mu$ of $S=(D$ or $\mathbf{C})-\left(\right.$ a discrete set) for $T\left(r, e^{f} \circ \mu\right)$, with $f \in \mathscr{H}(S)$ having arbitrary singularities. For example, what is the growth order of $T\left(e^{f} \circ \mu\right)$ for $\mu: D \rightarrow D-\{0\}$ with arbitrary $f \in \mathscr{H}(D-\{0\})$ ? Or, the growth order of $T\left(r, e^{f} \circ \mu\right)$ for $\mu: D \rightarrow \mathbf{C}-\{0,1\}$ with $f \in \mathscr{M}(\mathbf{C})$ ? 
Lemma 2. Let $U$ be 1-dimensional, $f \in \mathscr{F}(U)-\{0\}$, and $Y_{f}=\{$ the zeros of $f\} \cup\{$ the singularities of $f\}$. Then the Cantor derivative $Y_{f}^{(n)}=\varnothing$ for some $n \in \mathbf{N}$.

Recall that the Cantor derivatives of a closed set are defined inductively as follows: $Y^{(0)}=Y, Y^{(\alpha+1)}=$ the set of limit points of $Y^{(\alpha)}$, and $Y^{(\lambda)}=$ $\bigcap_{\alpha<\lambda} Y^{(\alpha)}$ for $\lambda$ a limit ordinal number.

Proof. Proceed by induction on the construction of $f$. If $f \in \mathscr{M}(U)-\{0\}$ then $Y_{f}$ is discrete and hence $Y_{f}^{(1)}=\varnothing$. If $f=g / h$ then $Y_{f} \subset Y_{g} \cup Y_{h}$ and hence $Y_{f}^{(n)}=\varnothing$ whenever $Y_{g}^{(n)}=Y_{h}^{(n)}=\varnothing$. If $f=e^{g}$ then $Y_{f} \subset Y_{g}$. If $f=g-h$ then $Y_{f} \subset Y_{g} \cup Y_{h} \cup A$, where $A$ (the set of zeros of $f$ ) has no limit points outside $Y_{g} \cup Y_{h}$; then $Y_{f}^{(1)} \subset Y_{g} \cup Y_{h}$ and hence $Y_{f}^{(n+1)}=\varnothing$ whenever $Y_{g}^{(n)}=Y_{h}^{(n)}=\varnothing$.

Lemma 3. Let $f_{j}, g_{k} \in \mathscr{F}(D) \cap \mathscr{H}(D-\{0\})$ and $p_{j}, q_{k} \in \mathscr{M}(D)-\{0\}$ for $1 \leq j \leq m, 1 \leq k \leq n$. Let $f_{j}-f_{j^{\prime}} \notin \mathscr{H}(D)$ for $j \neq j^{\prime}$ and $g_{k}-g_{k^{\prime}} \notin \mathscr{H}(D)$ for $k \neq k^{\prime}$. Let all $p_{j}, q_{k}$ be of bounded Nevanlinna characteristic. Denote

$$
h=\frac{\sum_{j=1}^{m} p_{j} e^{f_{j}}}{\sum_{k=1}^{n} q_{k} e^{g_{k}}} \in \mathscr{M}(D-\{0\}) .
$$

(i) If $h$ has neither zeros nor poles on $D-\{0\}$ then $m=n$ and there exists a permutation $\sigma$ of $\{1, \ldots, m\}$ such that $h=\left(p_{j} / q_{\sigma(j)}\right) \cdot e^{f_{j}-g_{\sigma(j)}}$ for all $j$.

(ii) If $T(r, h \circ \mu)=o\left(e^{2 / 1-r} \cdot(1-r)^{1.5}\right)$ as $r \rightarrow 1$ then $h \in \mathscr{M}(D), m=n$, and there exists a permutation $\sigma$ of $\{1, \ldots, m\}$ such that $f_{j}-g_{\sigma(j)} \in \mathscr{H}(D)$ and $h=\left(p_{j} / q_{\sigma(j)}\right) \cdot e^{f_{j}-g_{\sigma(j)}}$ for all $j$.

Proof. Denote $F_{0}=\mathscr{M}(D)$ and $F_{n}=$ the subfield of $\mathscr{E}(D)$ generated by $F_{n-1} \cup\left\{e^{f} \mid f \in F_{n-1}\right\}$. For $f \in \mathscr{F}(D)$ denote $\nu(f)=\min \left\{n \mid f \in F_{n}\right\}$. To prove the results proceed by induction on the maximum of $\left\{\nu\left(f_{j}\right)\right\}_{j}$ and $\left\{\nu\left(g_{k}\right)\right\}_{k}$. Denote $t(r)=e^{2 /(1-r)} \cdot(1-r)^{1.5}$.

Induction base. Let $f_{j}, g_{k} \in \mathscr{M}(D)$. Then all $f_{j}-f_{j^{\prime}}$ for $j \neq j^{\prime}$ and $g_{k}-g_{k^{\prime}}$ for $k \neq k^{\prime}$ have $0 \in D$ as a pole. By Lemma $1, T\left(r,\left(e^{f_{j}-f_{j^{\prime}}}\right) \circ \mu\right)$ for $j \neq j^{\prime}$ and $T\left(r,\left(e^{g_{k}-g_{k^{\prime}}}\right) \circ \mu\right)$ for $k \neq k^{\prime}$ are $>$ const $\cdot t(r)$ as $r \rightarrow 1$. On the other hand, all $T\left(r, p_{j} \circ \mu\right)$ and $T\left(r, q_{k} \circ \mu\right)$ are bounded (since all $p_{j}$ and $q_{k}$ are of bounded Nevanlinna characteristic, they are quotients of bounded analytic functions, and hence all $p_{j} \circ \mu$ and $q_{k} \circ \mu$ are quotients of bounded analytic functions). Now Corollary 3 of $\S 1.3$ implies that $m=n$ and that there exists a permutation $\sigma$ of $\{1, \ldots, m\}$ such that $h=\left(p_{j} / q_{\sigma(j)}\right) \cdot e^{f_{j}-g_{\sigma(j)}}$ for all $j$. If $f_{j}-g_{\sigma(j)} \notin \mathscr{H}(D)$ then $T\left(r,\left(e^{f_{j}-g_{\sigma(j)}}\right) \circ \mu\right)>$ const $\cdot t(r)$ by Lemma 1 and hence $T(r, h \circ \mu)>$ const $\cdot t(r)$ (recall that $T\left(r,\left(p_{j} / q_{\sigma(j)}\right) \circ \mu\right)$ is bounded). Thus, in case $T(r, h \circ \mu)=o(t(r))$ we have $f_{j}-g_{\sigma(j)} \in \mathscr{H}(D)$ for all $j$.

Induction step. Let $N=$ the maximum of $\left\{\nu\left(f_{j}\right)\right\}_{j}$ and $\left\{\nu\left(g_{k}\right)\right\}_{k}$. Then all those members of the family $\left\{f_{j}\right\}_{j} \cup\left\{g_{k}\right\}_{k}$ which are not meromorphic on $D$ can be presented in the form $(*)$ with all exponents occurring belonging to $F_{N-1}$. Hence all nonmeromorphic members of the family $\left\{f_{i}-f_{j}\right\}_{i \neq j} \cup$ $\left\{g_{k}-g_{l}\right\}_{k \neq l}$ can be presented in the form $(*)$ with all exponents occurring belonging to $F_{N-1}$. By the induction assumption then $T\left(r,\left(f_{i}-f_{j}\right) \circ \mu\right) \neq$ 
$o(t(r))$ whenever $f_{i}-f_{j} \notin \mathscr{M}(D)$ and $T\left(r,\left(g_{k}-g_{l}\right) \circ \mu\right) \neq o(t(r))$ whenever $g_{k}-g_{l} \notin \mathscr{M}(D)$. Note that $T(r, u)=o\left(T\left(e^{u}\right)\right)$ whenever $u$ is a holomorphic function of unbounded Nevanlinna characteristic (this is a very special case of a result of Clunie, see [17]). Hence $T\left(r,\left(e^{f_{i}-f_{j}}\right) \circ \mu\right) \neq o(t(r))$ whenever $f_{i}-f_{j} \notin \mathscr{M}(D)$ and $T\left(r,\left(e^{g_{k}-g_{l}}\right) \circ \mu\right) \neq o(t(r))$ whenever $g_{k}-g_{l} \notin \mathscr{M}(D)$. Besides, $T\left(r,\left(e^{f_{i}-f_{j}}\right) \circ \mu\right)>$ const $\cdot t(r)$ for $f_{i}-f_{j} \in \mathscr{M}(D)-\mathscr{H}(D)$ and $T\left(r,\left(e^{g_{k}-g_{l}}\right) \circ \mu\right)>$ const $\cdot t(r)$ for $g_{k}-g_{l} \in \mathscr{M}(D)-\mathscr{H}(D)$ by Lemma 1 . As before, all $T\left(r, p_{j} \circ \mu\right)$ and $T\left(r, q_{k} \circ \mu\right)$ are bounded.

Again, Corollary 3 of $\S 1.3$ implies that $m=n$ and that there exists a permutation $\sigma$ of $\{1, \ldots, m\}$ such that $h=\left(p_{j} / q_{\sigma(j)}\right) \cdot e^{f_{j}-g_{\sigma(j)}}$ for all $j$. Suppose that $f_{j}-g_{\sigma(j)} \notin \mathscr{H}(D)$ for some $j$. If $f_{j}-g_{\sigma(j)} \in \mathscr{M}(D)-\mathscr{H}(D)$ then $T\left(r,\left(e^{\left.f_{j}-g_{\sigma(j)}\right)} \circ \mu\right)>\right.$ const $\cdot t(r)$ by Lemma 1 . If $f_{j}-g_{\sigma(j)} \notin \mathscr{M}(D)$ then $f_{j}-g_{\sigma(j)}$ can be presented in the form $(*)$ with all exponents occurring belonging to $F_{N-1}$, and $T\left(r,\left(e^{f_{j}-g_{\sigma(j)}}\right) \circ \mu\right) \neq o(t(r))$ by the induction assumption. Consequently $T(r, h \circ \mu) \neq o(t(r))$ (recall that each $T\left(r,\left(p_{j} / q_{\sigma(j)}\right) \circ \mu\right)$ is bounded).

Now we are ready to prove Proposition 2 of the previous section. Consider all triples $(A, B, \tau)$, where $A$ and $B$ are partitions of $\{1, \ldots, m\}$ and $\{1, \ldots$, $n\}$ respectively into the same number of sets (i.e., $|A|=|B|$ ) and $\tau$ is a bijection $A \rightarrow B$ such that

$$
h=\frac{\sum_{j \in a} p_{j} e^{f_{j}}}{\sum_{k \in \tau(a)} q_{k} e^{g_{k}}}
$$

for each $a \in A$. We will write $(A, B, \tau) \preceq\left(A^{\prime}, B^{\prime}, \tau^{\prime}\right)$ if the triple $\left(A^{\prime}, B^{\prime}, \tau^{\prime}\right)$ is a refinement of the triple $(A, B, \tau)$, i.e., if every $a^{\prime} \in A^{\prime}$ is contained in some $a \in A$, every $b^{\prime} \in B^{\prime}$ is contained in some $b \in B$ and $\tau(a)=b$ whenever $\tau^{\prime}\left(a^{\prime}\right)=b^{\prime}$ for some $a^{\prime} \subset a, b^{\prime} \subset b$. The relation $\preceq$ is an ordering; let $(A, B, \tau)$ be a $\preceq$-maximal triple. We are going to show that both $A$ and $B$ consist of one-element sets only.

Suppose the contrary: $a \in A, b \in B, \tau(a)=b$, and $|a|+|b|>2$. Denote by $Y$ the set of singularities of all $f_{j}-f_{j^{\prime}}$ and $g_{k}-g_{k^{\prime}}$ for $j, j^{\prime} \in a$ and $k, k^{\prime} \in b$.

By Lemma 2 there exists an isolated point $u$ in $Y$. Take a closed disk neighborhood $V$ of $u$ in $U$ so small that $(V-\{u\}) \cap Y=\varnothing$. One can identify $(V, u)$ with the closed unit disk and its center $(\bar{D}, 0)$ in $\mathbf{C}$. Then all $\left\{p_{j}\right\}_{j \in a}$ and $\left\{q_{k}\right\}_{k \in b}$ are quotients of bounded analytic functions. Let $\alpha$ be the partition of $a$ into the equivalence classes of the relation $j \simeq j^{\prime}$ defined as $f_{j}-f_{j^{\prime}} \in \mathscr{H}(\bar{D})$, and let $\beta$ be the partition of $b$ into the equivalence classes of the relation $k \approx k^{\prime}$ defined as $g_{k}-g_{k^{\prime}} \in \mathscr{H}(\bar{D})$. Note that if $|a|>1$ then $|\alpha|>1$, if $|b|>1$ then $|\beta|>1$, and consequently $|\alpha|+|\beta|>2$. For each $J \in \alpha$ let $f_{J}$ be one of $\left\{f_{j}\right\}_{j \in J}$ and for each $K \in \beta$ let $g_{K}$ be one of $\left\{g_{k}\right\}_{k \in K}$. Put $\tilde{p}_{J}=\sum_{j \in J} p_{j} e^{f_{j}-f_{J}}$ and $\tilde{q}_{K}=\sum_{k \in K} q_{k} e^{g_{k}-g_{K}}$. Then all $\tilde{p}_{J}$ and $\tilde{q}_{K}$ are quotients of bounded analytic functions because $f_{j}-f_{J}, g_{k}-g_{K} \in \mathscr{H}(\bar{D})$, and

$$
h=\frac{\sum_{J \in \alpha} \tilde{p}_{J} e^{f_{J}}}{\sum_{K \in \beta} \tilde{q}_{K} e^{g_{K}}} .
$$

Now, by Lemma 3 , the number of sets in $\alpha$ and $\beta$ coincide, $|\alpha|=|\beta|$, and 
there exists a bijection $\sigma: \alpha \rightarrow \beta$ such that

$$
h=\frac{\tilde{p}_{J} e^{f_{J}}}{\tilde{q}_{\sigma(J)} e^{g_{\sigma(J)}}}=\frac{\sum_{j \in J} p_{j} e^{f_{j}}}{\sum_{k \in \sigma(J)} q_{k} e^{g_{k}}},
$$

for all $J \in \alpha$. Thus one can refine $(A, B, \tau)$ by taking

$$
((A-\{a\}) \cup \alpha,(B-\{b\}) \cup \beta,(\tau-\{(a, b)\}) \cup \sigma),
$$

i.e., replacing each $a \in A$ and $b \in B$ with several smaller sets and adding the correspondence $\sigma$ between the new sets. The latter contradicts the $\preceq$ maximality of $(A, B, \tau)$.

Lemma 4. Let $U$ be a connected complex-analytic manifold and $a \in \mathscr{F}(U)-$ $\mathscr{H}(U)$. Then there exist a finite set $X_{a} \subset U$ and an exceptional set $E_{a} \subset U$ such that $a \in \mathscr{H}\left(U-E_{a}\right)$ and $a \circ \psi \in \mathscr{F}(D)-\mathscr{H}(D)$ for any holomorphic embedding $\psi: D \rightarrow U$ satisfying $\psi(D) \supset X_{a}$ and $\psi(D)-E_{a} \neq \varnothing$.

Proof. Denote $F_{0}=\mathscr{M}(U)$ and $F_{n}=$ the subfield of $\mathscr{E}(U)$ generated by $F_{n-1} \cup\left\{e^{f} \mid f \in F_{n-1}\right\}$. For $f \in \mathscr{F}(U)$ denote $\nu(f)=\min \left\{n \mid f \in F_{n}\right\}$. To prove the result proceed by induction on $\nu(a)$.

Induction base. For $a \in \mathscr{M}(U)-\{0\}$ put $E_{a}=$ the polar set of $a$; pick a point $t$ in the polar set but not in the zero set of $a$ and put $X_{a}=\{t\}$. Let us show that these $X_{a}, E_{a}$ satisfy the requirements. Let $\psi: D \rightarrow U$ be a holomorphic embedding and $\psi(D) \supset X_{a}, \psi(D)-E_{a} \neq \varnothing$. Since the intersection $\psi(D) \cap E_{a}$ is an analytic subset of $\psi(D)$, it is discrete. For every $z \in D$ there exist functions $u, v$ holomorphic in a neighborhood of $\psi(z)$ in $U$ with the zero set of $v$ contained in $E_{a}$ such that $a=u / v$. Since $\psi(D) \cap E_{a}$ is discrete, none of $u \circ \psi$ and $v \circ \psi$ is identically zero (in a neighborhood of $z \in D)$ and $a \circ \psi=(u \circ \psi) /(v \circ \psi)$. Thus $a \circ \psi$ is meromorphic and not identically zero on $D$. Let $t=\psi(w)$. Since $t$ is a polar point but not a zero point of $a$, in a neighborhood of $t$ in $U$ one has $a=u / v$ for some holomorphic $u, v$ with $u(t) \neq 0$ and $v(t)=0$. Then

$$
(a \circ \psi)(z)=(u \circ \psi)(z) /(v \circ \psi)(z) \rightarrow \infty,
$$

as $z \rightarrow w$.

Induction step. Let $N>0$ and assume that the statement of the lemma holds whenever $\nu(a)<N$. Let $\nu(a)=N$. Then

$$
a=\frac{\sum_{j} p_{j} e^{f_{j}}}{\sum_{k} q_{k} e^{q_{k}}}
$$

for some $p_{j}, q_{k} \in \mathscr{M}(U)-\{0\}$ and $f_{j}, g_{k} \in F_{N-1}$ such that $f_{i}-f_{j} \notin \mathscr{H}(U)$ for $i \neq j$ and $g_{k}-g_{l} \notin \mathscr{H}(U)$ for $k \neq l$.

Consider first the case when each of $\sum_{j}$ and $\sum_{k}$ consists of one summand only. Then $a=(p / q) \cdot e^{f-g}$, where $f-g \notin \mathscr{H}(U)$ because otherwise $e^{f-g} \in$ $\mathscr{H}(U)$ and $a \in \mathscr{M}(U)$. Put $X_{a}=X_{f-g}$ and $E_{a}=E_{f-g} \cup\{$ the zero and polar points of $p / q\}$ (recall that $f-g \in F_{N-1}$ ). Let us show that these $X_{a}, E_{a}$ satisfy the requirements. Let $\psi: D \rightarrow U$ be a holomorphic embedding and $\psi(D) \supset X_{a}, \psi(D)-E_{a} \neq \varnothing$. We have $p / q, f-g \in \mathscr{H}\left(U-E_{a}\right)$, whence $(p / q) \cdot e^{f-g} \in \mathscr{H}\left(U-E_{a}\right)$. Further, $(p / q) \circ \psi \in \mathscr{M}(D)-\{0\}$ (see the reasoning in the induction base), and $(f-g) \circ \psi \notin \mathscr{H}(D)$ by the induction 
assumption. Consequently $e^{(f-g) \circ \psi}$ and $a \circ \psi=((p / q) \circ \psi) \cdot e^{(f-g) \circ \psi}$ are not only nonholomorphic but even nonmeromorphic on $D$ (see, e.g., Lemma 3 ).

Now consider the case when $a$ cannot be presented as $r \cdot e^{s}$ for $r \in \mathscr{M}(U)-$ $\{0\}$ and $s \in F_{N-1}$. Then at least one of $\sum_{j}, \sum_{k}$ above contains more than one summand, and the zero set of $a-\left(p_{j} / q_{k}\right) \cdot e^{f_{i}-g_{l}}$ is an analytic subset in $U-\left(\bigcup_{j} E_{f_{j}}\right)-\left(\bigcup_{k} E_{g_{k}}\right)$ for all $i, l$. Put

$$
X_{a}=\left(\bigcup_{j} X_{f_{j}}\right) \cup\left(\bigcup_{k} X_{g_{k}}\right) \cup\left(\bigcup_{i \neq j} X_{f_{i}-f_{j}}\right) \cup\left(\bigcup_{k \neq l} X_{g_{k}-g_{l}}\right),
$$

and

$$
\begin{aligned}
E_{a}= & \left(\bigcup_{j} E_{f_{j}}\right) \cup\left(\bigcup_{k} E_{g_{k}}\right) \cup\left(\text { the zero set of } \sum_{k} q_{k} e^{g_{k}}\right) \\
& \left.\cup \text { (the union of zero and polar sets of }\left\{p_{j}\right\}_{j}\right) \\
& \left.\cup \text { (the union of zero and polar sets of }\left\{q_{k}\right\}_{k}\right) \\
& \cup\left(\text { the union of zero sets of }\left\{a-\left(p_{j} / q_{k}\right) \cdot e^{f_{j}-g_{k}}\right\}_{j, k}\right) .
\end{aligned}
$$

Trivially, $a \in \mathscr{H}\left(U-E_{a}\right)$. Let $\psi: D \rightarrow U$ be a holomorphic embedding satisfying $\psi(D) \supset X_{a}, \psi(D)-E_{a} \neq \varnothing$. Then $f_{j} \circ \psi, g_{k} \circ \psi \in \mathscr{F}(D)$ by the induction assumption, $p_{j} \circ \psi, q_{k} \circ \psi \in \mathscr{M}(D)$ and so $a \circ \psi \in \mathscr{F}(D)$. Let us show that $a \circ \psi \notin \mathscr{H}(D)$. By the induction assumption $\left(f_{i}-f_{j}\right) \circ \psi \notin \mathscr{H}(D)$ for $i \neq j$ and $\left(g_{k}-g_{l}\right) \circ \psi \notin \mathscr{H}(D)$ for $k \neq l$. By Lemma 3, if $a \circ \psi \in \mathscr{H}(D)$ then there are $\sigma$ and $\tau$ such that $a \circ \psi=\left(\left(p_{\sigma} / q_{\tau}\right) \cdot e^{f_{\sigma}-g_{\tau}}\right) \circ \psi$, i.e., $\psi(D)$ is contained in the zero set of $a-\left(p_{\sigma} / q_{\tau}\right) \cdot e^{f_{\sigma}-g_{\tau}}$ (contradicting $\left.\psi(D)-E_{a} \neq \varnothing\right)$.

Now we are ready to prove Theorems 1 and 3 of the previous section. Note that for any finite set $A \subset U$ there exists a holomorphic embedding $\psi: D \rightarrow U$ with $\psi(D) \supset A$. Indeed, let $\psi^{\prime \prime}:[0,1] \rightarrow U$ be a $C^{\infty}$-path through all points of $A$ without critical points and self-intersections. By Lemma 1 of $\S 2.1$ one can substitute for $\psi^{\prime \prime}$ a real-analytic $\psi^{\prime}:[0,1] \rightarrow U$ with the same properties. Then $\psi^{\prime}$ can be analytically continued to an embedding $\psi$ of a neighborhood of $[0,1]$ into $U$.

In the notation of Theorems 1 and 3 of $\S 2.2$, put $X=\left(\bigcup_{j \neq j^{\prime}} X_{f_{j}-f_{j^{\prime}}}\right) \cup$ $\left(\bigcup_{k \neq k^{\prime}} X_{g_{k}-g_{k^{\prime}}}\right)$ and $E=E_{h} \cup\left(\bigcup_{j} E_{f_{j}}\right) \cup\left(\bigcup_{k} E_{g_{k}}\right) \cup$ (the union of the zero and polar sets of all $p_{j}$ and $q_{k}$ ), where the $X_{\text {? }}$ 's and $E_{\text {? }}$ 's are as described in Lemma 4 above. Consider all holomorphic embeddings $\psi$ of the closed disk $\bar{D}$ into $U$ satisfying $\psi(D) \supset X$ and $\psi(D)-E \neq \varnothing$. For each such $\psi$ the set of singularities of $h \circ \psi$ is contained in a proper analytic subset of $\bar{D}$, i.e., is finite. By Proposition 2 of $\S 2.2$ (proved above) then $m=n$ and there exists a permutation $\sigma=\sigma_{\psi}$ of $\{1, \ldots, m\}$ such that $h \circ \psi=\left(\left(p_{j} / q_{\sigma(j)}\right) \cdot e^{\left.f_{j}-g_{v(j)}\right) \circ \psi}\right.$ for all $j$. Since one can construct such $\psi$ passing through any prescribed point, for every $x \in U-E$ there exists a permutation $\sigma_{x}$ of $\{1, \ldots, m\}$ such that $h(x)=\left(\left(p_{j} / q_{\sigma_{x}(j)}\right) \cdot e^{\left.f_{j}-g_{\sigma_{x}(j)}\right)(x)}\right.$ for all $j$. Since there are only finitely many permutations of $\{1, \ldots, m\}$ whereas $U-E$ is open, there exists a $\sigma$ such that the identity $h(x)=\left(\left(p_{j} / q_{\sigma(j)}\right) \cdot e^{f_{j}-g_{\sigma(j)}}\right)(x)$ holds on an open subset of $U$ and hence on the whole of $U$. This proves Theorem 3 . 
For Theorem 1 of $\S 2.2$ we have to show that $f_{j}-g_{\sigma(j)} \in \mathscr{H}(U)$. Suppose the contrary: $f_{j}-g_{\sigma(j)} \notin \mathscr{H}(U)$. Then for an appropriate holomorphic embedding (see Lemma 4) $\psi: D \rightarrow U$ we have $\left(f_{j}-g_{\sigma(j)}\right) \circ \psi \in \mathscr{F}(D)-\mathscr{H}(D)$ and $\left(h \cdot q_{\sigma(j)} / p_{j}\right) \circ \psi \in \mathscr{M}(D)$. Then $\left(f_{j}-g_{\sigma(j)}\right) \circ \psi$ is a logarithm on $D$ of a meromorphic function $\left(h \cdot q_{\sigma(j)} / p_{j}\right) \circ \psi$, whereas $\log ($ a meromorphic function $s$ on $D$ ) either does not exist (if $s$ has a zero or a pole on $D$ ) or is holomorphic on $D$.

Next is the proof of Theorem 4 of $\S 2.2$. Proceed by induction on $n$. The case $n=1$ is almost proved in the example in $\S 2.0$ (in that example $U \subset \mathbf{C}$ ). Let $\left\{s_{j}\right\}_{j=0}^{m} \subset S_{0}$ be distinct. Without loss of generality one can assume that $s_{0}=0$. Let

$$
1=e^{s_{0}}=\sum_{j=1}^{m} p_{j} e^{s_{j}}=\sum_{j=1}^{m} p_{j} e^{s_{j}} / 1,
$$

for some $p_{j} \in \mathscr{M}(U)$. By Theorem 1 of $\S 2.2$ then $m=1$ and $s_{1} \in \mathscr{H}(U)$, contradicting the assumption on $S_{0}$.

Consider $n>1$. We have to show that for any $\left\{s_{j}\right\}_{j=1}^{m} \subset F_{n}$ satisfying $s_{k}-s_{l} \notin F_{n-1}$ for $k \neq l$, the functions $\left\{e^{s_{j}}\right\}_{j=0}^{m}$ are linearly independent over $F_{n-1}$. Note that each $F_{n}$ can be described also as the subfield of $\mathscr{E}(U)$ generated by $\mathscr{M}(U) \cup \exp \left(F_{n-1}\right)$, where $\exp \left(F_{n-1}\right)=\left\{e^{f} \mid f \in F_{n-1}\right\}$. Denote by $R_{n}$ the subring of $\mathscr{E}(U)$ generated by $\mathscr{M}(U) \cup \exp \left(F_{n-1}\right)$. To show that $\left\{e^{s_{j}}\right\}_{j}$ are linearly independent over $F_{n-1}$ it suffices to show that they are linearly independent over $R_{n-1}$. Without loss of generality one can assume that $s_{0}=0$. Let

$$
1=e^{s_{0}}=\sum_{j=1}^{m} q_{j} e^{s_{j}}=\sum_{j=1}^{m} q_{j} e^{s_{j}} / 1,
$$

where $q_{j} \in R_{n-1}-\{0\}$. Let $q_{j}=\sum_{k=1}^{l_{j}} p_{j k} e^{r_{j k}}$ with $p_{j k} \in \mathscr{M}(U)-\{0\}$ and $r_{j k} \in F_{n-2}$ for each $j$. Without loss of generality one can assume that $r_{j, k}-$ $r_{j, k^{\prime}} \notin \mathscr{H}(U)$ for $k \neq k^{\prime}$. For $j \neq j^{\prime}$ we have $\left(s_{j}-s_{j^{\prime}}\right)+\left(r_{j k}-r_{j^{\prime} k^{\prime}}\right) \in$ $F_{n-1}-F_{n-2} \subset F_{n-1}-\mathscr{H}(U)$. By Theorem 1 of $\S 2.2$ then $\sum_{j=1}^{m} l_{j}=1$, whence $m=1$ and $s_{1}+r_{11} \in \mathscr{H}(U)$, contradicting the assumption on $\left\{s_{j}\right\}_{j}$.

\section{EASY MULTIVALUED FUNCTIONS}

This is also an introductory section, but it is somewhat less introductory than $\S 1$. Here we consider mostly the rings of analytic germs (for every connected complex-analytic manifold $U$ and a point $\zeta \in U$ ) generated by the germs of the form $h=f^{g}$, where $f, g \in \mathscr{M}(U) \cap \mathscr{H}_{\zeta}$ and $f(\zeta) \neq 0$. The monodromy of such multivalued functions, i.e., how the result $\tilde{h}_{\lambda}$ of the analytic continuation of a germ $h$ along a loop $\lambda$ is related to $g$, is clear.

Reminders. $\mathscr{H}_{\zeta}^{*}=\{$ those holomorphic germs at $\zeta$ which have value $\neq 0$ at $\zeta\}, \mathscr{C}^{*}(U)=\{$ functions holomorphic on $U$ without zeros $\}$.

$H_{1}(X)=$ the first homology group of $X$. Note that (the $n=1$ case of) the Hurewicz homomorphism $\pi_{1}(X) \rightarrow H_{1}(X)$ is onto and its kernel is the commutant $\left[\pi_{1}(X), \pi_{1}(X)\right]$. Thus one can think of $H_{1}$ as $\left[\pi_{1}, \pi_{1}\right]$. For homomorphisms of groups $h: A \rightarrow B$, we use Bourbaki words "epimorphism" 
(onto), "monomorphism" $(h(x)=h(y) \Rightarrow x=y)$, and the notation im $h=$ $h(A), \operatorname{ker} h=h^{-1}(0)$.

The bar over a field means its algebraic closure; thus $\overline{\mathscr{M}}(U)=$ the algebroid functions on $U\}, \overline{\mathscr{E}}(U)=$ the algebraic closure of the field of functions holomorphic almost everywhere on $U$.

3.1. Lemmata. This subsection contains some basics on logarithms of holomorphic functions on connected complex-analytic manifolds (to be used in the sequel).

Lemma 1. Let $Q_{0}=P, Q_{1}, \ldots, Q_{k}$ be Weierstrass polynomials ${ }^{10}$ with respect to $z_{n}$ in a neighborhood $U$ of 0 in $\mathbf{C}^{n}$. Let the images of $\left\{Q_{j}\right\}_{j}$ as germs at 0 be distinct irreducibles in $\mathscr{H}_{0}$. Then there exists $a u \in U$ such that $Q_{1}, \ldots, Q_{k} \in \mathscr{H}_{u}^{*}$ and $u$ is an order 1 zero of $P$ (i.e., $P(u)=0$ and $\left(\partial P / \partial z_{j}\right)(u) \neq 0$ for at least one $\left.j, 1 \leq j \leq n\right)$.

Proof (following [33]). Put $g=\prod_{j} Q_{j}$. Since $g$ is not divisible by $P$ we have $g=P q+r$, where $q \in \mathscr{H}_{0}, r$ is a nonzero polynomial in $z_{n}$ with coefficients in $\mathscr{H}_{0}$ on $\mathbf{C}^{n-1}$, and $\operatorname{deg}(r)<\operatorname{deg}(P)$. Let $d$ be the resultant of $P$ and $\partial P / \partial z_{n}$. Since $P$ is irreducible, $d$ (which belongs to $\mathscr{H}_{0}$ on $\mathbf{C}^{n-1}$ ) does not vanish identically. Let $V$ be a neighborhood of 0 in $\mathbf{C}^{n-1}$ such that $d$ and the coefficients of $r$ are in $\mathscr{H}(V)$, and put $V_{*}=\{v \in V \mid d(v) \neq 0$ and at least one of the coefficients of $r$ is $\neq 0$ at $v\}$. Then $V_{*}$ is open and dense in $V$ and for any sufficiently small $v \in V_{*}$ the polynomial $P\left(z_{n}\right)$ has $\operatorname{deg}(P)$ distinct roots whereas $r$ has $\leq \operatorname{deg}(r)<\operatorname{deg}(P)$ roots. So there are $v \in V_{*}$ and $w$ such that $P(v, w)=0 \neq g(v, w)$. Since $w$ is a simple root of $P(v, \cdot)$ the order of $P$ in $\mathscr{H}_{(v, w)}$ is 1 and thus $P$ is an irreducible in $\mathscr{H}_{(v, w)}$.

If $U$ is a complex analytic manifold and $f \in \mathscr{H}^{*}(U)$ then the increment of $\frac{1}{2 \pi} \arg f$ along a loop (the same as the increment of $\frac{1}{2 \pi i} \log f$ along a loop) is a homomorphism $\pi_{1}(U) \rightarrow \mathbf{Z}$ and hence a homomorphism $H_{1}(U) \rightarrow \mathbf{Z}$.

Lemma 2. Let $U$ be a neighborhood of 0 in $\mathbf{C}^{n}, P \in \mathscr{H}(U)$, and 0 be an order 1 zero of $P$. Let $\zeta \in U, P(\zeta) \neq 0$. Then the homomorphism

$$
\frac{1}{2 \pi} \arg P: H_{1}(U-Y, \zeta) \rightarrow \mathbf{Z},
$$

where $Y$ is the zero set of $P$, is an epimorphism.

Proof. Denote by $D$ the unit disk of C. For $v \in \mathbf{C}^{n}-\{0\}$ denote by $\psi_{v}$ the embedding $\mathbf{C} \rightarrow \mathbf{C}^{n}$ given by $w \mapsto v w$. Take a $v \in \mathbf{C}^{n}$ such that $\psi_{v}(D) \subset U$ and 0 is the only root and a simple root of $P \circ \psi_{v}$ on $D$. Then

$$
\frac{1}{2 \pi} \arg \left(P \circ \psi_{v}\right): H_{1}(D-\{0\}, \zeta) \rightarrow \mathbf{Z}
$$

is an isomorphism, whence

$$
\frac{1}{2 \pi} \arg P: H_{1}\left(\psi_{v}(D)-\{0\}, \zeta\right) \rightarrow \mathbf{Z}
$$

\footnotetext{
${ }^{10} \mathrm{~A}$ Weierstrass polynomial with respect to $z_{n}$ around $\overrightarrow{0} \in \mathbf{C}^{n}$ is $\left(z_{n}\right)^{k}+c_{1}\left(z_{n}\right)^{k-1}+\cdots+$ $c_{k-1} z_{n}+c_{k}$, where each $c_{j}$ is a function in $\left(z_{1}, \ldots, z_{n-1}\right)$ holomorphic in a neighborhood of $\overrightarrow{0}$ in $\mathbf{C}^{n-1}$ and $c_{j}(\overrightarrow{0})=0$.
} 
is an isomorphism too. The result then follows from $\psi_{v}(D)-\{0\} \subset U-Y$.

Lemma 3. Let $Q_{1}, \ldots, Q_{k} \in \mathscr{H}(U)$, where $U$ is a neighborhood of 0 in $\mathbf{C}^{n}$, $\zeta \in U-\{0\}$, and $Q_{j}(\zeta) \neq 0$ for all $j$. Let the images of $\left\{Q_{j}\right\}_{j}$ be distinct irreducibles in $\mathscr{H}_{0}, Q_{l} / Q_{m} \notin \mathscr{H}_{0}^{*}$ for $l \neq m$. Let $Y$ be the union of the zero sets of $\left\{Q_{j}\right\}_{j}$. Then the family $\left\{\nu_{j}\right\}_{j=1}^{k}$, where

$$
\nu_{j}=\frac{1}{2 \pi} \arg Q_{j}: H_{1}(U-Y, \zeta) \rightarrow \mathbf{Z},
$$

maps $H_{1}(U-Y, \zeta)$ onto $\mathbf{Z}^{k}$.

Proof. By Lemmas 1 and 2 for each $j$ there exists a loop $\gamma$ in $(U-Y, \zeta)$ such that $\nu_{j}(\gamma)=1$ while $\nu_{m}(\gamma)=0$ for $m \neq j$.

Lemma 4. Let $U$ be a connected complex-analytic manifold, $K$ be an exceptional subset of $U$, and $\zeta \in U-K$. Let $f \in \mathscr{H}^{*}(U-K)$. If $\frac{1}{2 \pi} \arg f$ maps $H_{1}(U-K, \zeta)$ into $\{0\}$ then there exists a $g \in \mathscr{H}(U-K)$ such that $f=e^{g}$. If, besides that, $f \in \mathscr{M}(U)$ then $g \in \mathscr{H}(U)$.

Proof. Take for $g$ any branch of $\log f$. For $f \in \mathscr{M}(U)$ it suffices to show that $f$ has neither zeros nor poles in $U$. Suppose the contrary: the germ of $f$ at some $u \in U$ has the decomposition $\prod_{j=1}^{k}\left(\psi_{j}\right)^{l_{j}}$, where $l_{j} \in \mathbf{Z}-\{0\}, k>0$, and all $\left\{\psi_{j}\right\}_{j}$ are distinct irreducibles of $\mathscr{H}_{u}$ (in particular, $\psi_{j} / \psi_{j^{\prime}} \notin \mathscr{H}_{u}^{*}$ for $j \neq j^{\prime}$ ). Then the homomorphism

$$
\left\{\frac{1}{2 \pi} \arg \psi_{j}\right\}_{j}: H_{1}(U-Y, \zeta) \rightarrow \mathbf{Z}^{k},
$$

where $Y$ is the zero and pole sets of $f$, is an epimorphism. Since

$$
\pi_{1}(U-K, \zeta) \rightarrow \pi_{1}(U-Y, \zeta)
$$

is an epimorphism,

$$
\frac{1}{2 \pi} \arg f=\frac{1}{2 \pi} \sum_{j=1}^{k} l_{j} \cdot \arg \psi_{j}: H_{1}(U-K, \zeta) \rightarrow \mathbf{Z}
$$

does not vanish.

Let $U$ be a connected complex-analytic manifold and $\zeta \in U$.

Lemma 5. Let $f_{j} \in \mathscr{M}(U) \cap \mathscr{H}_{\zeta}^{*}$ and $g_{j} \in \overline{\mathscr{E}}(U) \cap \mathscr{H}_{\zeta}$ for $1 \leq j \leq k$. Fix a value for $\log f_{j}(\zeta)$ for each $j$. If $1, g_{1}, \ldots, g_{k}$ are linearly independent over $\mathbf{Q}$ and the germ $\prod_{j=1}^{k}\left(f_{j}\right)^{g_{j}} \in \overline{\mathscr{E}}(U)$ then $\left\{\log f_{j}\right\}_{j} \subset \mathscr{H}(U)$.

Proof. Let an exceptional subset $K \subset U, \zeta \notin K$, and a finite-sheeted covering $(V, \zeta) \rightarrow(U-K, \zeta)$ be such that all $g_{j} \in \mathscr{H}(V)$. The increment of $\frac{1}{2 \pi i} \log \left(\prod_{j=1}^{k}\left(f_{j}\right)^{g_{j}}\right)=\frac{1}{2 \pi i} \sum_{j=1}^{k} g_{j} \log f_{j}$ along a loop in $(V, \zeta)$ is equal to the increment of $\frac{1}{2 \pi} \sum_{j=1}^{k} g_{j} \arg f_{j}$ along a loop in $(V, \zeta)$ and is a homomorphism $\pi_{1}(V, \zeta) \rightarrow \mathscr{H}(V)$ with values in $\mathbf{Z}$. The linear independence of $\{1\} \cup\left\{g_{j}\right\}_{j}$ over $\mathbf{Q}$ implies that all $\frac{1}{2 \pi} \arg f_{j}$ map $\pi_{1}(V, \zeta)$ into $\{0\}$. Since the index of $\pi_{1}(V, \zeta)$ in $\pi_{1}(U-K, \zeta)$ is finite, all $\frac{1}{2 \pi} \arg f_{j}$ map $\pi_{1}(U-K, \zeta)$ into $\{0\}$, and Lemma 4 provides the result. 
Lemma 6. Let $f_{j} \in \mathscr{M}(U) \cap \mathscr{H}_{\zeta}^{*}$ and $g_{j} \in \mathscr{H}(U)$ for $1 \leq j \leq k$. Fix a value for $\log f_{j}(\zeta)$ and put $h_{j}=\left(f_{j}\right)^{g_{j}}$ for each $j$. If for some exceptional set $K$ the increment of $\frac{1}{2 \pi} \sum_{j} g_{j}$ arg $f_{j}$ along a loop maps $\pi_{1}(U-K, \zeta)$ into the copy of $\mathbf{Z}$ in $\mathscr{H}(U-K)$ consisting of integer constants then $h=\prod_{j} h_{j} \in \mathscr{M}(U)$.

Proof. Let $m$ and $k^{\prime} \leq k$ be such that each $g_{j}$ for $k^{\prime}<j \leq k$ is an integer linear combination of $1 / m, g_{1} / m, \ldots, g_{k^{\prime}} / m$ and $1, g_{1}, \ldots, g_{k^{\prime}}$ are linearly independent over $\mathbf{Q}$. Then $h=\left(\tilde{f}_{0}\right)^{1 / m} \cdot \prod_{j=1}^{k^{\prime}}\left(\tilde{f}_{j}\right)^{g_{j} / m}$ for some $\left\{\tilde{f}_{j}\right\}_{j=0}^{k^{\prime}} \subset \mathscr{M}(U)$, and the increment of $\frac{1}{2 \pi i} \log h$ along a loop, $\frac{1}{2 \pi m} \arg \tilde{f}_{0}+\frac{1}{2 \pi m} \sum_{j=1}^{k^{\prime}} g_{j} \arg \tilde{f}_{j}$, maps $H_{1}(U-K, \zeta)$ into $\mathrm{Z} \subset \mathscr{H}(U-K)$ for some exceptional set $K$. Since $1, g_{1}, \ldots, g_{k^{\prime}}$ are linearly independent over $\mathbf{Q}$, each $\frac{1}{2 \pi} \arg \tilde{f}_{j}, 1 \leq j \leq k^{\prime}$, maps $H_{1}(U-K, \zeta)$ into $\{0\}$ and $\frac{1}{2 \pi} \arg \tilde{f}_{0}$ maps $H_{1}(U-K, \zeta)$ into $m \mathbf{Z}$. Hence $\left\{\log \tilde{f}_{j}\right\}_{j=1}^{k^{\prime}} \subset \mathscr{H}(U)$ by Lemma 4 , and $\left(\tilde{f}_{0}\right)^{1 / m} \in \mathscr{M}(U)$.

3.2. Examples. Let $U$ be a connected complex-analytic manifold; fix a point $\zeta \in U$. Let $f_{j} \in \mathscr{M}(U) \cap \mathscr{H}_{\zeta}^{*}$ and $g_{j} \in \mathscr{H}(U)$ for $1 \leq j \leq k$. Fix a value for $\log f_{j}(\zeta)$ and put $h_{j}=\left(f_{j}\right)^{g_{j}} \in \mathscr{H}_{\zeta}^{*}$ for each $j$. In this subsection we show how easy multivaluedness considerations help to detect algebraic or analytic (in)dependence of $\left\{h_{j}\right\}_{j}$.

Consider first algebraic relations between the $h_{j}$ 's. For any multi-index $\alpha \in$ $\mathbf{Z}^{k}$ denote $t_{\alpha}=\prod_{j=1}^{k}\left(h_{j}\right)^{\alpha_{j}}$. An algebraic relation between the $h_{j}$ 's over a field $\mathscr{F}$ is a linear relation between some $t_{\alpha}$ 's over $\mathscr{F}$. The examples below which concern algebraic dependence of $\left\{h_{j}\right\}_{j}$ or linear dependence of $\left\{t_{\alpha}\right\}_{\alpha}$ are some vague analogues of the Hiromi-Ozawa lemma for "exponentials" $\left\{t_{\alpha}\right\}_{\alpha}$ with "Nevanlinna-small" and "Nevanlinna-large" substituted by "few-valued" and "many-valued" respectively.

Example 1. Let all $f_{j} \in \mathscr{H}^{*}(U)$. Take a finite family $\left\{t_{\alpha}\right\}_{\alpha \in A}$, and consider homomorphisms

$$
\nu_{\alpha}=\sum_{j} \frac{\alpha_{j}}{2 \pi} g_{j} \arg f_{j}: \pi_{1}(U, \zeta) \rightarrow \mathscr{H}(U),
$$

for each $\alpha \in A$ (each $\nu_{\alpha}$ is "the increment of $\frac{1}{2 \pi i} \log t_{\alpha}$ along a loop"). If there exists a $\tau \in \pi_{1}(U, \zeta)$ such that $\left(\nu_{\beta}-\nu_{\gamma}\right)(\tau) \notin \mathbf{Z}$ for $\beta \neq \gamma, \beta, \gamma \in A$, then $\left\{t_{\alpha}\right\}_{\alpha \in A}$ are linearly independent over $\mathscr{E}(U)$.

Indeed, suppose that $\sum_{\alpha \in A} c_{\alpha} t_{\alpha}=0$ with some $c_{\alpha} \in \mathscr{E}(U)$. Let $K \subset U$ be an exceptional subset such that all $c_{\alpha} \in \mathscr{H}(U-K)$. Without loss of generality one can assume that $\zeta \notin K$ (otherwise one could substitute $\zeta$ with a point in $U-K$ sufficiently close to $\zeta)$. Let $\lambda$ be a loop in $(U-K, \zeta)$ homotopic to $\tau$ in $(U, \zeta)$. The analytic continuation along $\lambda^{m}, m \in \mathbf{N}$, multiplies each $c_{\alpha} t_{\alpha}$ by $e^{2 \pi i m \nu_{\alpha}(\tau)}$ but the whole sum must remain 0 :

$$
\sum_{\alpha \in A} c_{\alpha} t_{\alpha} e^{2 \pi i m \nu_{\alpha}(\tau)}=0 .
$$

These identities for $0 \leq m \leq N-1$ ( $N=$ the number of elements of $A$ ) can be regarded as a homogenous system of linear equations for the germs $\left\{c_{\alpha} t_{\alpha}\right\}_{\alpha \in A}$ in $\mathscr{M}_{\zeta}$ with Vandermonde matrix

$$
\left\{\left(\eta_{p}\right)^{q}\right\}_{p, q=0}^{N-1}
$$


where $\left\{\eta_{p}\right\}_{p=0}^{N-1}$ is an enumeration of $\left\{e^{2 \pi i \nu_{\alpha}(\tau)}\right\}_{\alpha \in A}$. By the assumption on $\tau, e^{2 \pi i \nu_{\beta}(\tau)} \neq e^{2 \pi i \nu_{\gamma}(\tau)}$ whenever $\beta \neq \gamma$, i.e., all $\eta_{p}$ are distinct. Hence the determinant

$$
\left|\left\{\left(\eta_{p}\right)^{q}\right\}_{p, q=0}^{N-1}\right| \neq 0
$$

and the system considered has unique solution with all "unknowns" equal to 0 . Since each $t_{\alpha} \in \mathscr{H}_{\zeta}^{*}$ this means that each $c_{\alpha}=0$.

In the above example the requirement that a single loop $\tau$ makes all the differences $\nu_{\beta}-\nu_{\gamma}$ nonintegral is excessively strong. The result can be generalized as follows.

Example 2. In the notation of Example 1, if $\operatorname{im}\left(\nu_{\beta}-\nu_{\gamma}\right) \not \subset \mathbf{Z}$ for $\beta \neq \gamma$, $\beta, \gamma \in A$, then $\left\{t_{\alpha}\right\}_{\alpha \in A}$ are linearly independent over $\mathscr{E}(U)$.

If you dare to take exp (a homomorphism from a group into a function germs field) then you can delete all occurrences of $\tau$ from the proof above, and then regard what will remain as a proof of the current statement. (This idea is not as wild as it may seem to be; we actually use it in the continuation of this paper.) Here is a conventional proof of the statement of Example 2.

Let $\left\{c_{\alpha}\right\}_{\alpha \in A} \subset \mathscr{E}(U)$ and $\sum_{\alpha \in A} c_{\alpha} t_{\alpha}=0$. Consider nonempty subsets $B \subset$ $A$ minimal with respect to the property $\sum_{\alpha \in B} c_{\alpha} t_{\alpha}=0$. It is enough to show that each such $B$ is a one-point set.

Suppose the contrary: $\beta, \gamma \in B$ and $\beta \neq \gamma$. Let $K$ be an exceptional subset of $U$ such that all $c_{\alpha} \in \mathscr{H}(U-K)$. Without loss of generality one can assume that $\zeta \notin K$. Let $\tau$ be a loop in $(U, \zeta)$ such that $\left(\nu_{\beta}-\nu_{\alpha}\right)(\tau) \notin \mathbf{Z}$ and $\lambda$ be a loop in $(U-K, \zeta)$ homotopic to $\tau$ in $(U, \zeta)$. Let us break $B$ into the equivalence classes $\left\{B_{\sigma}\right\}_{\sigma=0}^{l}$ determined by the relation $\simeq_{\tau}$, where $\alpha \simeq_{\tau} \alpha^{\prime}$ is defined as $\left(\nu_{\alpha}-\nu_{\alpha^{\prime}}\right)(\tau) \in \mathbf{Z}$. Then $l \geq 1$ (there are at least two equivalence classes: $\left.\beta \not_{\tau} \gamma\right)$. Put $u_{\sigma}=\sum_{\alpha \in B_{\sigma}} c_{\alpha} t_{\alpha}$ for $0 \leq \sigma \leq l$; then

$$
\sum_{\sigma=0}^{l} u_{\sigma}=0 \text {. }
$$

The analytic continuation along the loop $\lambda^{m}, m \in \mathbf{N}$, multiplies each $u_{\sigma}$ by $e^{2 \pi i m \nu_{\alpha}(\tau)}$, where $\alpha \in B_{\sigma}$, but the whole sum must remain zero:

$$
\sum_{\sigma=0}^{l} u_{\sigma} \cdot\left(\eta_{\sigma}\right)^{m}=0,
$$

where $\eta_{\sigma}=e^{2 \pi i \nu_{\alpha}(\tau)}, \alpha \in B_{\sigma}$, for each $\sigma$. Note that $\eta_{\sigma} \neq \eta_{\sigma^{\prime}}$ for $\sigma \neq \sigma^{\prime}$. Now the same Vandermonde matrix argument as before shows that each $u_{\sigma}=0$ (contradicting the assumption on $B$ ).

One can further refine Example 2 as follows.

Example 3. In the notation of Example 1, break $A$ into the equivalence classes $\left\{A_{\sigma}\right\}_{\sigma}$ determined by the relation $\simeq$, where $\alpha \simeq \alpha^{\prime}$ is defined as $\operatorname{im}\left(\nu_{\alpha}-\nu_{\alpha^{\prime}}\right) \subset$ Z. If $\left\{c_{\alpha}\right\}_{\alpha \in A} \subset \mathscr{E}(U)$ and $\sum_{\alpha \in A} c_{\alpha} t_{\alpha}=0$ then $\sum_{\alpha \in A_{\sigma}} c_{\alpha} t_{\alpha}=0$ for each $\sigma$; more precisely, if $B$ is a nonempty subset of $A$ minimal with respect to the property $\sum_{\alpha \in B} c_{\alpha} t_{\alpha}=0$ then $B$ is contained in some $A_{\sigma}$.

The latter statement is proved exactly as in Example 2 (note that the equivalence relation $\simeq$ is the intersection of the equivalence relations $\simeq_{\tau}, \tau \in$ $\left.\pi_{1}(U, \zeta)\right)$. 
These facts allow one to reduce the questions on algebraic dependence of $\left\{h_{j}\right\}_{j}$ over $\mathscr{E}(U)$ to questions about meromorphic functions on $U$, via the following.

Remark 4. If $\left\{h_{j}\right\}_{j=1}^{k}$ are algebraically dependent over $\mathscr{E}(U)$ then there exists an $\alpha \in \mathbf{Z}^{k}-\{0\}$ such that $t_{\alpha} \in \mathscr{M}(U)$.

Indeed, let some finite family $\left\{t_{\alpha}\right\}_{\alpha \in A}$ be linearly dependent over $\mathscr{E}(U)$, i.e., $\sum_{\alpha \in A} c_{\alpha} t_{\alpha}=0$, all $c_{\alpha} \in \mathscr{E}(U)$ and at least one $c_{\alpha} \neq 0$. Then Example 3 says that at least one $\simeq$-equivalence class $A_{\sigma}$ contains more than one element: $\beta, \gamma \in A_{\sigma}$ and $\beta \neq \gamma$. Then $\nu_{\beta-\gamma}=\nu_{\beta}-\nu_{\gamma}$ maps $\pi_{1}(U, \zeta)$ into $\mathbf{Z}$, and the statement (with $\alpha=\beta-\gamma \in \mathbf{Z}-\{0\}$ ) follows from Lemma 6 of $\S 3.1$.

The above remark says that if there exists a nonzero polynomial $P \in$ $\mathscr{E}(U)\left[z_{1}, \ldots, z_{k}\right]$ with $P\left(\left\{h_{j}\right\}_{j=1}^{k}\right)=0$ then there exists a 2-nomial $Q \in$ $\mathscr{M}(U)\left[\left\{z_{j}\right\}_{j=1}^{k}\right]$ with $Q\left(\left\{h_{j}\right\}_{j=1}^{k}\right)=0$. (A 2-nomial is a polynomial consisting of exactly two monomials.)

Let us consider how it can happen that $t_{\alpha} \in \mathscr{M}(U)$ for some $\alpha \in \mathbf{Z}^{k}$ $-\{0\}$. Let $1, s_{1}, \ldots, s_{l}$ be a Q-linear basis for the Q-linear span of $1, g_{1}, \ldots$, $g_{k}$, and $m \in \mathbf{N}$ be such that each $g_{j}$ is an integer linear combination of $\{1 / m\} \cup\left\{s_{i} / m\right\}_{i=1}^{l}$ (when all functions $\left\{g_{j}\right\}_{j}$ are presented explicitly it is often easy to find such $\left.1, s_{1}, \ldots, s_{l}\right)$. Then each $t_{\alpha}$ can be presented as $\left(\tilde{f}_{0}\right)^{1 / m}$. $\prod_{i=1}^{l}\left(\tilde{f}_{i}\right)^{s_{i} / m}$, where all $\tilde{f}_{i} \in \mathscr{M}(U)$ are of the form $\prod_{j=1}^{k}\left(f_{j}\right)^{n_{j}}$ with appropriate $n_{i} \in \mathbf{Z}$. By Lemma 5 for such $t_{\alpha}$ to be in $\overline{\mathscr{E}}(U)$ it is necessary and sufficient that $\left\{\log \tilde{f}_{i}\right\}_{i=1}^{l} \subset \mathscr{H}(U)$. To get $t_{\alpha} \in \mathscr{M}(U)$ the latter condition has to be augmented only with $\left(\tilde{f}_{0}\right)^{1 / m} \in \mathscr{M}(U)$. In view of this, in the remaining part of this subsection we shall consider only the case $1, g_{1}, \ldots, g_{k}$ linearly independent over $\mathbf{Q}$. Then the statements in some previously considered examples can be formulated more strongly.

Example 5. Let $1, g_{1}, \ldots, g_{k}$ be linearly independent over $\mathbf{Q}$. If $\left\{t_{\alpha}\right\}_{\alpha \in A}$ are linearly independent over $\overline{\mathscr{E}}(U)$ then $\log \left(t_{\beta} / t_{\gamma}\right) \in \mathscr{H}(U)$ for some $\beta \neq \gamma$, $\beta, \gamma \in A$.

In this example the multivaluedness of the $t_{\delta}$ 's (each of them is either infinitely multivalued or possesses a logarithm on $U$, see Lemma 5 of $\S 3.1$ ) is compared with that of the coefficients of a linear relation, which are assumed to be finitely multivalued. Recall also that a $t_{\delta}$ possesses a logarithm on $U$ if and only if all $\left\{f_{j} \mid \delta_{j} \neq 0\right\}$ possess logarithms on $U$.

To prove the statement suppose that $\left\{c_{\alpha}\right\}_{\alpha \in A} \subset \overline{\mathscr{E}}(U)$, at least one $c_{\alpha} \neq 0$ and $\sum_{\alpha \in A} c_{\alpha} t_{\alpha}=0$. One can assume (upon changing $\zeta$, if necessary) that there are an exceptional set $K \subset U, \zeta \notin K$, and a finite-sheet covering $(V, \zeta) \rightarrow$ $(U-K, \zeta)$ such that all $c_{\alpha} \in \mathscr{H}(V)$ and all $f_{j} \in \mathscr{H}^{*}(U-K)$. Denote

$$
\mu_{\alpha}=\sum_{j} \frac{\alpha_{j}}{2 \pi} g_{j} \arg f_{j}: \pi_{1}(V, \zeta) \rightarrow \mathscr{H}(V) .
$$

Consider nonempty subsets $B \subset A$ minimal with respect to the property $\sum_{\alpha \in B} c_{\alpha} t_{\alpha}=0$. Since at least one $c_{\alpha} \neq 0$, there exists such $B$ containing more than one element. Let $\beta, \gamma \in B$ and $\beta \neq \gamma$. In view of Example 3 then $\operatorname{im}\left(\mu_{\beta-\gamma}\right) \subset \mathbf{Z}$. Denote $\delta=\beta-\gamma$. Since all $\frac{\delta_{j}}{2 \pi} \arg f_{j}$ have integer values and 
$1, g_{1}, \ldots, g_{k}$ are linearly independent over $\mathbf{Q}$, all $\frac{\delta_{j}}{2 \pi} \arg f_{j} \operatorname{map} \pi_{1}(V, \zeta)$ into $\{0\}$. Since $\pi_{1}(V, \zeta)$ is of finite index in $\pi_{1}(U-K, \zeta)$, all $\frac{\delta_{j}}{2 \pi} \arg f_{j}$ map $\pi_{1}(U-K, \zeta)$ into $\{0\}$. Hence $\sum_{j=1}^{k} \frac{\delta_{j}}{2 \pi} g_{j} \arg f_{j}$ maps $\pi_{1}(U-K, \zeta)$ into $\{0\}$. Then $t_{\delta} \in \mathscr{M}(U)$ by Lemma 6 of $\S 3.1$ and $\log t_{\delta} \in \mathscr{H}(U)$ by Lemma 5 of $\S 3.1$.

The next example is a combination of multivaluedness considerations and the Hiromi-Ozawa lemma.

Example 6. Let $U=\mathbf{C}^{n}$ and let $1, g_{1}, \ldots, g_{k}$ be linearly independent over $\mathbf{Q}$. If $\left\{t_{\alpha}\right\}_{\alpha \in A}$ are linearly dependent over $\mathbf{C}(z)$ then $t_{\beta} / t_{\gamma}=$ const for some $\beta \neq \gamma, \beta, \gamma \in A$.

Let all $q_{\alpha} \in \mathbf{C}(z)$, at least one $q_{\alpha} \neq 0$, and $\sum_{\alpha \in A} q_{\alpha} t_{\alpha}=0$. In view of Example 3 there exists $B \subset A$ containing at least two elements such that $\log \left(t_{\beta} / t_{\gamma}\right) \in \mathscr{H}\left(\mathbf{C}^{n}\right)$ for $\beta, \gamma \in B$ and $\sum_{\alpha \in B} q_{\alpha} t_{\alpha}=0$. Let $u$ be one of $\left\{t_{\alpha}\right\}_{\alpha \in B}$. Then $\sum_{\alpha \in B} q_{\alpha} \cdot\left(t_{\alpha} / u\right)=0$ and each $t_{\alpha} / u$ has the form $e^{\psi_{\alpha}}$ for some $\psi_{\alpha} \in \mathscr{H}\left(\mathbf{C}^{n}\right)$. Thus it remains only to apply the Hiromi-Ozawa lemma to $\sum_{\alpha \in B} q_{\alpha} e^{\psi_{\alpha}}=0$.

The latter example hints at how the multivaluedness considerations can be applied to Tarski's high school algebra problem.

3.3. An application to Tarski's high school algebra problem. Denote $\mathscr{S}_{1}=$ the closure of $\{a \uparrow b \mid a, b \in \Sigma\}$ in $L$ under addition and multiplication, and $\mathscr{L}_{1}=$ the closure of $\{a \uparrow b \mid a, b \in \Lambda\}$ in $L$ under addition and multiplication. These $\mathscr{S}_{1}$ and $\mathscr{L}_{1}$ are the next (after $\Sigma$ and $\Lambda$ themselves) slices of the classes $\mathscr{S}=\{t \in L \mid$ if $a \uparrow b$ occurs as a subterm of $t$ then $a \in \Sigma\}$ and $\mathscr{L}=\{t \in L \mid$ if $a \uparrow b$ occurs as a subterm of $t$ then $a \in \Lambda\}$. Classes $\Sigma$ ("the Schanuel class") and $\Lambda$ ("the Levitz class") were considered in [19]; see also $\S 1.4$ of this paper. (Sometimes we shall refer to them as $\mathscr{S}_{0}$ and $\mathscr{L}_{0}$ respectively.) Later in this paper we shall consider the whole of the classes $\mathscr{S}$ and $\mathscr{L}$, but in this subsection we only want to present an easy example of how multivaluedness considerations are applied to Tarski's high school algebra problem. Clearly

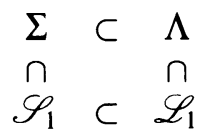

but no more inclusions hold between these classes.

Examples (in the variables $x, y, z$ ). (i) $2^{2^{2^{x}}+y \cdot 3^{z}}+x z \in \Sigma$. (ii) $x^{y^{z}} \in \Lambda-\mathscr{P}_{1}$. (iii) $\left(2^{x}+y\right)^{z} \in \mathscr{S}_{1}-\Lambda$. (iv) $\left(2^{x}+y^{z}\right)^{y^{x}} \in \mathscr{L}_{1}-\left(\Lambda \cup \mathscr{S}_{1}\right)$.

Recall from $\S 1.4$ that both $\bar{\Sigma}$ and $\bar{\Lambda}$ are free semirings with respect to addition and multiplication; their generators (the $(+, \cdot)$-primes sans 1$)$ are described in Corollary 2 and Corollary 4 of $\S 1.4$. Each $f \in \overline{\mathscr{S}}_{1}$ is a sum of +-primes of $\overline{\mathscr{S}_{1}}$ and every +-prime of $\overline{\mathscr{S}}_{1}$ can be presented in the form (see $\left.\S 1.2\right)$

(a monic monomial)

$$
\begin{aligned}
& \times \prod_{i}(\text { a monomial, } \neq 1)_{i} \uparrow(\mathrm{a}+\text {-prime } \in \bar{\Sigma}, \neq 1)_{i} \\
& \times \prod_{j}(\text { a function } \in \bar{\Sigma}, \neq 1, \text { with content }=1)_{j} \\
& \uparrow(\text { a }+ \text {-prime } \in \bar{\Sigma}, \neq 1)_{j},
\end{aligned}
$$


perhaps with empty $\prod_{i}$ or $\prod_{j}$ (or both), with all exponents occurring in $\prod_{i}$ distinct and all exponents occurring in $\prod_{j}$ distinct.

Proposition 1. (i) Let $f, g$ possess representations of the form $\left(Q_{1}\right)$. If $\mathrm{f} / \mathrm{g}=$ const then $f=g$ and their $\left(Q_{1}\right)$-representations coincide up to a permutation of the factors.

(ii) Let $f_{1}, \ldots, f_{k} \in \overline{\mathscr{S}}_{1}$ have the form $\left(\mathcal{O}_{1}\right)$. If $f_{l} \neq f_{m}$ for $l \neq m$ then $\left\{f_{l}\right\}_{l}$ is linearly independent over $\mathbf{R}$.

Proof. (i) Let $f=u \cdot \prod_{i}\left(p_{i}\right)^{q_{i}} \cdot \prod_{j}\left(r_{j}\right)^{s_{j}}$ and $g=u^{\prime} \cdot \prod_{i}\left(p_{i}^{\prime}\right)^{q_{i}^{\prime}} \cdot \prod_{j}\left(r_{j}^{\prime}\right)^{s_{j}^{\prime}}$ as in $\left(Q_{1}\right)$. Let $\left\{\tau_{n}\right\}_{n}$ be an enumeration of $\left\{q_{i}\right\}_{i} \cup\left\{q_{i}^{\prime}\right\}_{i}$ (without repetitions) and $\left\{\lambda_{m}\right\}_{m}$ be an enumeration of $\left\{s_{j}\right\}_{j} \cup\left\{s_{j}^{\prime}\right\}_{j}$ (without repetitions). Then $f / g=\left(u / u^{\prime}\right) \cdot \prod_{n}\left(a_{n} / a_{n}^{\prime}\right)^{\tau_{n}} \cdot \prod_{m}\left(b_{m} / b_{m}^{\prime}\right)^{\lambda_{m}}=$ const, where

$$
a_{n}=\left\{\begin{array}{ll}
p_{i} & \text { if } q_{i}=\tau_{n} \text { for some } i, \\
1 & \text { otherwise, }
\end{array} \quad b_{m}= \begin{cases}r_{j} & \text { if } s_{j}=\lambda_{m} \text { for some } j, \\
1 & \text { otherwise },\end{cases}\right.
$$

and the definitions of $a_{n}^{\prime}, b_{m}^{\prime}$ differ from those of $a_{n}, b_{m}$ only by the strokes over $q_{i}, p_{i}$ and $s_{j}, r_{j}$ respectively. Recall that both families $\{1\} \cup\left\{\tau_{n}\right\}_{n}$ and $\{1\} \cup\left\{\lambda_{m}\right\}_{m}$ are linearly independent over $\mathbf{R}$ (see $\S 1.4$ ).

$\chi$ (see $\S 1.4)$ transforms $u / u^{\prime}$ and all $a_{n} / a_{n}^{\prime}$ into exponentials of entire functions. Thus

$$
\chi(f / g)=\exp (\text { an entire function }) \cdot \prod_{m}\left(\chi\left(b_{m}\right) / \chi\left(b_{m}^{\prime}\right)\right)^{\chi\left(\lambda_{m}\right)}=\text { const } .
$$

By Lemma 5 of $\S 3.1$ then all $\chi\left(b_{m}\right) / \chi\left(b_{m}^{\prime}\right)$ have neither zeros nor poles. Since all $r_{j}$ and $r_{j}^{\prime}$ have content $=1$, all $b_{m}$ and $b_{m}^{\prime}$ have content $=1$, and consequently all $\chi\left(b_{m}\right)$ and $\chi\left(b_{m}^{\prime}\right)$ have content $=1$ (see $\left.\S 1.4\right)$. By Proposition 5 of $\S 1.4$ then $\chi\left(b_{m}\right)=\chi\left(b_{m}^{\prime}\right)$ (whence $b_{m}=b_{m}^{\prime}$ ) for all $m$. Thus $\mathrm{f} / \mathrm{g}=$ $\left(u / u^{\prime}\right) \cdot \prod_{n}\left(a_{n} / a_{n}^{\prime}\right)^{\tau_{n}}=$ const. Recall that all $a_{n}$ and $a_{n}^{\prime}$ are monomials (not necessarily monic). By Lemma 5 of $\S 3.1$ then all $a_{n} / a_{n}^{\prime}$ have neither zeros nor poles. Hence $a_{n} / a_{n}^{\prime}=c_{n} \in \mathbf{Q}_{+}$for all $n$, and $f / g=\left(u / u^{\prime}\right) \cdot \prod_{n}\left(c_{n}\right)^{\tau_{n}}=$ const . Consequently $u=u^{\prime}$ (they are monic monomials and $u / u^{\prime}$ has neither zeros nor poles). Thus $f / g=\prod_{n}\left(c_{n}\right)^{\tau_{n}}=$ const. It remains to show that all $c_{n}=1$. We have $\log (f / g)=\sum_{n} \log \left(c_{n}\right) \cdot \tau_{n}=$ const. Since the family $\{1\} \cup\left\{\tau_{n}\right\}_{n}$ is linearly independent over $\mathbf{R}$, the latter identity is possible only if all $\log \left(c_{n}\right)=$ 0 .

(ii) For $1 \leq j \leq k$ let $u_{j}$ be the "monic monomial" part of $f_{j}$ in the $\left(O_{1}\right)$ form and $w_{j}=f_{j} / u_{j}$ (the remaining part of $f_{j}$ in the $\left(Q_{1}\right)$-form). Partition $\{1, \ldots, k\}$ into the equivalence classes $\left\{A_{\nu}\right\}_{\nu}$ of the relation $\simeq$, where $l \simeq m$ means $w_{l}=w_{m}$. Let $t_{\nu} \in\left\{w_{j} \mid j \in A_{\nu}\right\}$ for each $\nu$. Suppose that $f_{1}, \ldots, f_{k}$ are linearly dependent over $\mathbf{C}$; let $c_{j} \in \mathbf{C}$, at least one $c_{j} \neq 0$, and $\sum_{j=1}^{k} c_{j} f_{j}=$ 0 . Then $\sum_{\nu} q_{\nu} t_{\nu}=0$, where $q_{\nu}=\sum_{j \in A_{\nu}} c_{j} u_{j}$. Since the $f_{j}$ 's are all distinct, for each $\nu$ the monic monomials $\left\{u_{j}\right\}_{j \in A_{\nu}}$ are distinct. Consequently $q_{\nu} \neq 0$ whenever $c_{j} \neq 0$ for at least one $j \in A_{\nu}$. Hence at least one $q_{\nu} \neq 0$, and the $t_{\nu}$ 's are linearly dependent over $\mathbf{C}[z]$. In view of Example 6 of $\S 3.2$ there exist $\nu \neq \mu$ such that $t_{\nu} / t_{\mu}=$ const. Since each $t_{\rho}$ has the form $\left(O_{1}\right)$ (with the trivial "monic monomial" part) by the first part of the proposition $t_{\nu}=t_{\mu}$, contradicting the definition of $\simeq$. 
Remarks. (i) Let $g_{1}, \ldots, g_{n}$ be distinct + -primes of $\bar{\Sigma}$, all $\neq 1$. Let $b_{1}, \ldots$, $b_{n}$ belong to the group generated by the functions from $\bar{\Sigma}$ of content 1 , and $b_{j} \neq 1$ for all $j$. Then $\left(b_{1}\right)^{g_{1}}, \ldots,\left(b_{n}\right)^{g_{n}}$ are algebraically independent over the field of rational functions.

Suppose the contrary. Then by Example 6 of $\S 3.2$ there exist $\alpha_{1}, \ldots, \alpha_{n} \in \mathbf{Z}$, at least one of them nonzero, such that $\prod_{j=1}^{n}\left(\left(b_{j}\right)^{g_{j}}\right)^{\alpha_{j}}=$ const. Let $E=$ the union of the zero and polar sets of the $b_{j}$ 's. Consider

$$
\nu_{j}=\frac{1}{2 \pi} \arg b_{j}: \pi_{1}\left(\mathbf{C}^{m}-E\right) \rightarrow \mathbf{Z} .
$$

Each $b_{j}$ is a $\neq 1$ quotient of functions of content 1 and by the proposition

$$
\nu=\sum_{j=1}^{n} \alpha_{j} g_{j} \nu_{j}: \pi_{1}\left(\mathbf{C}^{m}-E\right) \rightarrow \mathscr{H}\left(\mathbf{C}^{m}\right)
$$

has $\operatorname{im}(\nu) \not \subset \mathbf{Z}$ because $1, g_{1}, \ldots, g_{n}$ are linearly independent and at least one $\alpha_{j} \neq 0$. Note that a constant function induces the abovementioned way the trivial $(=0)$ homomorphism $\pi_{1}\left(\mathbf{C}^{m}-E\right) \rightarrow \mathscr{H}\left(\mathbf{C}^{m}\right)$. Then by Example 1 of $\S 3.2, \prod_{j=1}^{n}\left(\left(b_{j}\right)^{g_{j}}\right)^{\alpha_{j}}$ and 1 are linearly independent.

(ii) The +-primes of $\overline{\mathscr{S}_{0}}$ are +-prime in $\overline{\mathscr{S}}_{1}$, and the $(+, \cdot)$-primes of $\overline{\mathscr{S}_{0}}$ are $(+, \cdot)$-prime in $\overline{\mathscr{S}_{1}}$. Indeed, the $\left(\nabla_{0}\right)$-form is an instance of the $\left(\nabla_{1}\right)$-form.

The class $\mathscr{L}_{1}$ is similar to the class $\mathscr{S}_{1}$ in the same way as $\Lambda\left(=\mathscr{L}_{0}\right)$ is similar to $\Sigma\left(=\mathscr{S}_{0}\right)$. Each $f \in \overline{\mathscr{L}}_{1}$ is a sum of t-primes of $\overline{\mathscr{L}_{1}}$ and every +-prime of $\overline{\mathscr{L}}_{1}$ can be presented in the form

(a monic monomial)

$$
\begin{aligned}
& \times \prod_{i}(\text { a monomial, } \neq 1)_{i} \uparrow(\mathrm{a}+\text {-prime } \in \bar{\Lambda}, \neq 1)_{i} \\
& \times \prod_{j}(\text { a function } \in \bar{\Lambda}, \neq 1, \text { with content }=1)_{j} \\
& \uparrow(\mathrm{a}+\text {-prime } \in \bar{\Lambda}, \neq 1)_{j},
\end{aligned}
$$

perhaps with empty $\prod_{i}$ or $\prod_{j}$ (or both), with all exponents occurring in $\prod_{i}$ distinct and all exponents occurring in $\Pi_{j}$ distinct. The uniqueness of this presentation is proved by reduction to that of $\left(\nabla_{1}\right)$ with the transformation $\chi_{\psi}$ described in $§ 1.4$.

Proposition 2. (i) $\chi\left\{\right.$ the +-primes of $\left.\overline{\mathscr{L}_{1}}\right\} \subset\left\{\right.$ the + -primes of $\left.\overline{\mathscr{S}_{1}}\right\}$ and $\chi\{$ the $(+, \cdot)$-primes of $\left.\overline{\mathscr{L}_{1}}\right\} \subset\left\{\right.$ the $(+, \cdot)$-primes of $\left.\overline{\mathscr{S}_{1}}\right\}$.

(ii) Let $f, g$ possess representations of the form $\left(\boldsymbol{\beta}_{1}\right)$. If $\mathrm{f} / \mathrm{g}=\mathrm{const}$ then $f=g$ and their $\left(\boldsymbol{\$}_{1}\right)$-representations coincide up to a permutation of multipliers.

(iii) Let $f_{1}, \ldots, f_{k} \in \overline{\mathscr{L}}_{1}$ have the form $\left(\boldsymbol{(}_{1}\right)$. If $f_{l} \neq f_{m}$ for $l \neq m$ then $\left\{f_{l}\right\}_{l}$ is linearly independent over $\mathbf{R}$.

Proof. It suffices to show that $\chi$ (every $\left(\boldsymbol{\beta}_{1}\right)$-form) can be presented by a $\left(\nabla_{1}\right)$ form and in such a way that the $\left(\nabla_{1}\right)$-forms corresponding to $\chi$ (distinct $\left(\boldsymbol{\beta}_{1}\right)$ forms) are distinct.

Consider $f=u \cdot \prod_{i}\left(p_{i}\right)^{q_{i}} \cdot \prod_{j}\left(r_{j}\right)^{s_{j}}$ in the $\left(\boldsymbol{\beta}_{1}\right)$-form. Let $u=x^{\alpha}$ and $p_{i}=$ $m_{i} x^{\beta_{i}}$, where $x=\left\{x_{t}\right\}_{t}$ is the family of the variables occurring, $m_{i} \in \mathbf{N}$, and 
$\alpha,\left\{\beta_{i}\right\}_{i}$ are multi-indices. Then $\chi(u)=\prod\left\{\left(a_{t}\right)^{x_{t}} \mid \alpha_{t} \neq 0\right\}$ and $\chi\left(\prod_{i}\left(p_{i}\right)^{q_{i}}\right)=$ $\prod\left\{\left(m_{i}\right)^{\chi\left(q_{i}\right)} \mid m_{i} \neq 1\right\} \cdot \prod\left\{\left(b_{i, t}\right)^{x_{t} \cdot x\left(q_{i}\right)} \mid i, t\right.$ with $\left.\beta_{i, t} \neq 0\right\}$, where $a_{t}=2^{\alpha_{t}}$ and $b_{i, t}=2^{\beta_{i, t}}$. By Proposition 3 of $\S 1.4$ all $\chi\left(r_{j}\right) \in \bar{\Sigma}-\{1\}$ and have content $=1$, and $\left\{\chi\left(s_{j}\right)\right\}_{j}$ are $\neq 1$ distinct + -primes of $\bar{\Sigma}$. Thus $\prod_{j}\left(\chi\left(r_{j}\right)\right)^{\chi\left(s_{j}\right)}$ makes up the $\prod_{j}$-part of the $\left(O_{1}\right)$-form of $\chi(f)$. To check that $\chi\left(u \cdot \prod_{i}\left(p_{i}\right)^{q_{i}}\right)=$ $\prod\left\{\left(a_{t}\right)^{x_{t}} \mid \alpha_{t} \neq 0\right\} \cdot \prod\left\{\left(m_{i}\right)^{x\left(q_{i}\right)} \mid m_{i} \neq 1\right\} \cdot \prod\left\{\left(b_{i, t}\right)^{x_{i} \cdot x\left(q_{i}\right)} \mid \beta_{i, t} \neq 0\right\}$ does make up the $\prod_{i}$-part of the $\left(Q_{1}\right)$-form of $\chi(f)$ it suffices to show that the +-primes $\left\{x_{t}\right\}_{t},\left\{\chi\left(q_{i}\right)\right\}_{i},\left\{x_{t} \cdot \chi\left(q_{i}\right)\right\}_{i, t}$ are all distinct. Note that all $\chi\left(q_{i}\right)$ are distinct because the $\left\{q_{i}\right\}_{i}$ are. Since all $\chi\left(q_{i}\right)$ are exponentials of entire functions (see Proposition 3 of $\S 1.4$ ), if $x_{t^{\prime}} \cdot \chi\left(q_{i^{\prime}}\right)=x_{t} \cdot \chi\left(q_{i}\right)$ then $t^{\prime}=t$ (compare the zero sets of both sides). Thus all $x_{t} \cdot \chi\left(q_{i}\right)$ are distinct. $\chi\left(q_{i^{\prime}}\right) \neq x_{t} \cdot \chi\left(q_{i}\right)$ since the left-hand side does not have zeros whereas the right-hand side does. $x_{t} \neq \chi\left(q_{i}\right)$ for the same reason. $x_{t^{\prime}} \neq x_{t} \cdot \chi\left(q_{i}\right):$ if $t^{\prime} \neq t$ then both sides have different zero sets, and for $t^{\prime}=t$ the inequality follows from $q_{i} \neq 1$.

Consider also $f^{\prime}=u^{\prime} \cdot \prod_{i}\left(p_{i}^{\prime}\right)^{q_{i}^{\prime}} \cdot \prod_{j}\left(r_{j}^{\prime}\right)^{s_{j}^{\prime}}$ in the $\left(\boldsymbol{q}_{1}\right)$-form. If $f$ and $f^{\prime}$ differ in the $\prod_{j}$-parts of their $\left(\boldsymbol{Q}_{1}\right)$-forms then the same difference occurs in the $\prod_{j}$-parts of the $\left(Q_{1}\right)$-forms of $\chi(f), \chi\left(f^{\prime}\right)$-see above. Thus it remains to consider the case when $f$ and $f^{\prime}$ have only the "monic monomial" and the $\prod_{i}$-parts in their $\left(\boldsymbol{\beta}_{1}\right)$-forms. Let $f$ and $f^{\prime}$ be such. Then $\chi(f)$ and $\chi\left(f^{\prime}\right)$ have only the $\prod_{i}$-parts in their $\left(O_{1}\right)$-forms. Suppose that the $\left(Q_{1}\right)$-forms of $\chi(f)$ and $\chi\left(f^{\prime}\right)$ coincide. Then $\left\{x_{t}\right\}_{t} \cup\left\{\chi\left(q_{i}\right)\right\}_{i} \cup\left\{x_{t} \cdot \chi\left(q_{i}\right)\right\}_{i, t}$ and $\left\{x_{t^{\prime}}\right\}_{t^{\prime}} \cup$ $\left\{\chi\left(q_{i^{\prime}}^{\prime}\right)\right\}_{i^{\prime}} \cup\left\{x_{t^{\prime}} \cdot \chi\left(q_{i^{\prime}}^{\prime}\right)_{i^{\prime}, t^{\prime}}\right\}$ are the same set. The comparison of the zero sets of its elements shows (as above) that $\left\{\chi\left(q_{i}\right)\right\}_{i}=\left\{\chi\left(q_{i^{\prime}}^{\prime}\right)\right\}_{i^{\prime}},\left\{x_{t}\right\}_{t}=\left\{x_{t^{\prime}}\right\}_{t^{\prime}},\left\{x_{t}\right.$. $\left.\chi\left(q_{i}\right)\right\}_{i, t}=\left\{x_{t^{\prime}} \cdot \chi\left(q_{i^{\prime}}^{\prime}\right)\right\}_{i^{\prime}, t^{\prime}}$, and $x_{t} \cdot \chi\left(q_{i}\right) \neq x_{t^{\prime}} \cdot \chi\left(q_{i^{\prime}}^{\prime}\right)$ unless $t^{\prime}=t$ and $q_{i^{\prime}}^{\prime}=q_{i}$. Consequently one can assume without loss of generality that $q_{i}=q_{i}^{\prime}$. Then $a_{t}^{\prime}=$ $a_{t}, \alpha_{t}^{\prime}=\alpha_{t}, m_{i}^{\prime}=m_{i}, b_{i, t}^{\prime}=b_{i, t}, \beta_{i, t}^{\prime}=\beta_{i, t}$, where $a_{t}^{\prime}, \alpha_{t}^{\prime}, m_{i}^{\prime}, b_{i, t}^{\prime}, \beta_{i, t}^{\prime}$ are defined for $f^{\prime}$ in the same way as $a_{t}, \alpha_{t}, m_{i}, b_{i, t}, \beta_{i, t}$ were defined for $f$. So, the $\left(\boldsymbol{\alpha}_{1}\right)$-forms of $f$ and $f^{\prime}$ coincide.

Corollary 3. (i) Let $g_{1}, \ldots, g_{n}$ be distinct +-primes of $\bar{\Lambda}$, all $\neq 1$. Let $b_{1}, \ldots, b_{n}$ belong to the group generated by the functions from $\bar{\Lambda}$ of content 1 , and all $b_{j} \neq 1$. Then $b_{1}^{g_{1}}, \ldots, b_{n}^{g_{n}}$ are algebraically independent over the field of rational functions.

(ii) The +-primes of $\bar{\Lambda}$ and those of $\overline{\mathscr{S}}_{1}$ are +-prime in $\overline{\mathscr{L}_{1}}$, and the (+, .)primes of $\bar{\Lambda}$ and those of $\overline{\mathscr{S}_{1}}$ are $(+, \cdot)$-prime in $\overline{\mathscr{L}_{1}}$.

\section{MEROMORPHIC AND OTHER FEW-VALUED FUNCTIONS AS BASES FOR EXPONENTIATION}

4.1. Introduction. Let $U$ be a connected complex-analytic manifold. Fix a point $\zeta \in U$. In this section we consider the germs at $\zeta$ obtained from fewvalued functions on $U$ by the ring operations and restricted exponentiation: $f, g \mapsto f^{g}$ is permitted only for $f$ few-valued and belonging to $\mathscr{H}_{\zeta}{ }^{*}$ and $g$ a previously obtained germ (or a few-valued function), $g \in \mathscr{H}_{\zeta}$.

Of course, "multivalued function on $U$ " is not a function on $U$; this expression refers to a holomorphic function on a (perhaps branched) covering of $U$ or to a germ in $\mathscr{H}_{\zeta}$ satisfying certain conditions. 
Definition. A germ $g \in \mathscr{H}_{\zeta}$ will be called global on $U$ (notation: $g \in \mathscr{G}_{\zeta}(U)$ or, shorter, $\left.g \in \mathscr{G}_{\zeta}\right)$ if the set of paths in $(U, \zeta)$ along which $g$ can be analytically continued is dense in the space of all paths in $(U, \zeta) .^{11}$

For example, each $g \in \mathscr{H}(U)$ is global trivially, each $g \in \mathscr{M}(U) \cap \mathscr{H}_{\zeta}$ is global because every path in $(U, \zeta)$ can be approximated by one not passing through the polar set of $g$.

Note that the set of paths in $(U, \zeta)$ along which a germ $g \in \mathscr{H}_{\zeta}$ can be analytically continued is always open. So, for a global germ $g \in \mathscr{\mathscr { H }}_{\zeta}$ the set of paths along which it cannot be analytically continued is closed and nowhere dense in the space of all paths in $(U, \zeta)$. This fact implies easily that the set of global germs at $\zeta$ is closed under many operations. It is obvious that $\mathscr{G}_{\zeta}$ is a ring, it is easy to see that $1 / g \in \mathscr{G}_{\zeta}$ for $g \in \mathscr{H}_{\zeta}^{*} \cap \mathscr{G}_{\zeta}$. Moreover, $\mathscr{G}_{\zeta}$ is relatively algebraically closed in $\mathscr{H}_{\zeta}$ : if $g \in \mathscr{H}_{\zeta}$ is algebraic over $\mathscr{G}_{\zeta}$ then $g \in \mathscr{G}_{\zeta}$ (it is still very easy to prove). It is not difficult to show that $\mathscr{G}_{\zeta}$ is relatively analytically closed in $\mathscr{H}_{\zeta}$ in the following sense: if $f \in \mathscr{H}_{\zeta}$ and $h\left(u, g_{1}, \ldots, g_{n}, f\right)=0$, where $h \in \mathscr{H}\left(U \times \mathbf{C}^{n+1}\right)-\{0\}, g_{j} \in \mathscr{G}_{\zeta}$, then $f \in \mathscr{G}_{\zeta}$. In particular, if $f \in \mathscr{G}_{\zeta}$, $f(\zeta) \neq 0$, then $\log f \in \mathscr{G}_{\zeta}$. We do not prove these properties of $\mathscr{G}_{\zeta}$ here because the germs we shall deal with in the sequel will be obviously global.

Informally, a global germ $g$ at $\zeta$ is a few-valued function on $U$ if $g$ can be continued along almost every loop $\lambda$ in $(U, \zeta)$, not merely along almost every path ("almost every" means except a closed nowhere dense subset of), and the set of the results $\left\{g_{\lambda}\right\}_{\lambda} \subset \mathscr{H}_{\zeta}$ of those analytic continuations is small in a sense. For example, $(x+1)^{2^{x}}$ is a global but not a few-valued germ (look at its analytic continuations around -1 ). If $g$ can be analytically continued along almost every loop in $(U, \zeta)$ and the result of each such continuation coincides with $g$ then (it is easy to see that) $g \in \mathscr{E}(U)$. Another example is: let $g \in \mathscr{H}_{\zeta}$ be algebraic over $\mathscr{M}(U)$ and $P \in(\mathscr{M}(U))[t]$ be its minimal polynomial. Then $g$ can be continued analytically along every loop in $(U, \zeta)$ not passing through the zero and polar sets of the coefficients and the discriminant of $P$, and the total number of germs at $\zeta$ which can be obtained from $g$ by those analytic continuations is not more than $\operatorname{deg}(P)$.

Let $\Phi \subset \mathscr{G}_{\zeta}$ be some class of few-valued functions on $U$, such as the field of algebroid function germs ${ }^{12}$ of $\mathscr{H}_{\zeta}$. Let $\mathbf{E}(U, \zeta)$ be the minimal subring of $\mathscr{H}_{\zeta}$ containing $\Phi$ and closed under the restricted exponentiation: $f, g \mapsto f^{g}$ for $f \in \Phi \cap \mathscr{H}_{\zeta}^{*}, g \in \mathbf{E}$. Germs in $\mathbf{E}(U, \zeta)$ can be continued analytically along almost every loop in $(U, \zeta)$ but usually are not few-valued. We analyze the monodromy phenomena in $\mathbf{E}$ (i.e., how the result $g_{\lambda} \in \mathscr{H}_{\zeta}$ of the analytic continuation of $g$ along $\lambda$ is related to $g$ ) in sufficient detail to prove results on algebraic (in)dependence of the functions mentioned in the Henson-Rubel conjecture, but not in great detail.

Suppose one is looking for algebraic relations between $g_{1}, \ldots, g_{m}$. Since only finitely many functions were used as bases for exponentiations in the construction of $\left\{g_{j}\right\}_{j}$ one can remove from $U$ the corresponding zero and polar sets and look for algebraic relations between $\left\{g_{j}\right\}_{j}$ on the remaining part $V$ of $U$. Further, one can take a finite-sheet covering $\theta:(W, \zeta) \rightarrow(V, \zeta)$ such that

\footnotetext{
${ }^{11} \mathrm{~A}$ path in $(U, \zeta)$ is a mapping $([0,1], 0) \rightarrow(U, \zeta)$. The space of paths in $(U, \zeta)$ is the set of all paths in $(U, \zeta)$ equipped with the compact-open topology.

${ }^{12}$ An algebroid function is an element of the algebraic closure $\mathscr{M}(U)$ of $\mathscr{M}(U)$.
} 
for each $f$ used in the construction of $\left\{g_{j}\right\}_{j}$ the corresponding $f \circ \theta$ is singlevalued on $W$. Now one can look for algebraic relations between $\left\{g_{j} \circ \theta\right\}_{j}$, and if there are none, then $\left\{g_{j}\right\}_{j}$ were also algebraically independent. Traditional monodromy considerations are closely related to what we do. Note that $\left\{g_{j} \circ \theta\right\}_{j}$ are some of the germs at $\zeta$ on $V$ obtained from functions holomorphic on $V$ by the ring operations and exponentiation restricted as follows: $f, g \mapsto f^{g}$ is permitted for $f \in \mathscr{H}^{*}(V)$ and $g$ a previously obtained germ (or $g \in \mathscr{H}(V))$. Let us denote by $T$ the set of all such germs, $T \subset \mathbf{E}(V, \zeta)$. Each $g \in T$ can be continued analytically along every loop in $(V, \zeta)$ and hence one has a representation of $\pi_{1}(V, \zeta)$ by linear transformations of $T$. A traditional complex variable theorist at this moment would ask various algebraic questions about this representation. Perhaps, the algebraic investigation of $\pi_{1}(V, \zeta) \rightarrow \mathrm{GL}(T, \mathscr{H}(V))$ would later provide results on algebraic relations between $\left\{g_{j}\right\}_{j}$. However we prefer (at least now) to go straight to our target.

The following result is an analogue of Remark 4 of $\S 3.2$.

Theorem 1. Put $S_{0}=\mathscr{H}_{\zeta} \cap \overline{\mathscr{M}}(U)$. For $n \in \mathbf{N}$ put $B_{n}=$ the multiplicative subgroup of $\mathscr{H}_{\zeta}^{*}$ generated by $\bigcup\left\{\right.$ the set of all branches of $f^{g} \mid f \in \mathscr{H}_{\zeta}^{*} \cap \overline{\mathscr{M}}(U)$ and $\left.g \in \mathscr{H}_{\zeta} \cap S_{n-1}\right\}$, and $S_{n}=$ the subring of $\mathscr{H}_{\zeta}$ generated by $S_{n-1} \cup B_{n}$.

Let $h_{0}, \ldots, h_{m} \in \bigcup_{n=1}^{\infty} B_{n}$. If $\left\{h_{j}\right\}_{j}$ are linearly dependent over $\mathscr{M}_{\zeta} \cap \overline{\mathscr{M}}(U)$ then there exist $j \neq k$ such that $h_{j} / h_{k} \in \overline{\mathscr{M}}(U)$.

Algebroid (vs. meromorphic) functions cannot be avoided in Theorem 1 even if one restricts exponentiation $f^{g}$ to $f \in \mathscr{H}_{\zeta}^{*} \cap \mathscr{M}(U)$. For example, $1, e^{z^{1 / 2}}, e^{-z^{1 / 2}}$ are linearly dependent over $\mathscr{M}(\mathbf{C}),{ }^{13}$ while any two of them are not.

The above theorem can be extended to a larger class of functions. Its proof (later in this section) is based on the following fact. If a germ $g \in \mathscr{H}_{\zeta} \cap$ $\overline{\mathscr{H}}(U)$ is continued analytically along loops $\lambda$ in $(U, \zeta)$ not passing through the branching points of the minimal polynomial of $g$, then there is $n=n(g) \in \mathbf{N}$ such that the analytic continuation of $g$ along $\lambda^{n}$ yields $g$ again for any $\lambda$. The class of germs with this property is already larger than $\overline{\mathscr{M}}$; consider, e.g.,

$$
\sum_{n=1}^{\infty} \frac{1}{n^{3}}(z-n)^{1 / 2} \in \mathscr{H}_{0}
$$

(this formula represents uncountably many similar germs, for one can choose the values of the roots arbitrarily). To see that each such germ $g$ is not algebroid note that $g$ can be continued along every loop in $\mathbf{C}-\mathbf{N}$ and these analytic continuations yield infinitely many germs in $\mathscr{H}_{0}$. Say, a loop winding once around $n$ and not winding around other positive integers returns the germ

$$
g_{n}=-\frac{(z-n)^{1 / 2}}{n^{3}}+\sum_{k \in \mathrm{N}-\{n\}} \frac{1}{k^{3}}(z-k)^{1 / 2},
$$

and $g_{n} \neq g_{m}$ for $n \neq m$. However, it is not necessary for the proof of the theorem to have $n$ depending on $g$ only; one can permit $n$ to depend on $\lambda$ too.

\footnotetext{
${ }^{13}$ Indeed, $e^{z^{1 / 2}}+e^{-z^{1 / 2}}=2 \cos (i \sqrt{z}) \in \mathscr{H}(\mathbf{C})$.
} 
Definition. Let $g \in \mathscr{M}_{\zeta}$ and let $\lambda$ be a loop in $(U, \zeta)$ such that $g$ can be continued analytically along $\lambda^{n}$ for all $n \in \mathbf{Z}$. Then $g$ will be called (non)periodic with respect to $\lambda$ if the function $\mathbf{Z} \rightarrow \mathscr{M}_{\zeta}$ defined as $k \mapsto$ (the analytic continuation of $g$ along $\lambda^{k}$ ) is (non)periodic.

Definition. Let $g \in \mathscr{M}_{\zeta}$. We shall write $g \in \mathscr{P}(U)$ or, shorter, $g \in \mathscr{P}$, if there exists an exceptional set $K \subset U, \zeta \notin K$, such that $g$ can be continued along and is periodic with respect to every loop in $(U-K, \zeta)$.

For example, if $g \in \mathscr{M}_{\zeta}$ is algebraic over $\mathscr{M}(U)$ then $g$ is periodic with respect to every loop not passing through the branching points of $g$. The following are some germs in $\mathscr{H}_{0}$ on $\mathbf{C}$ which belong to $\mathscr{P}-\overline{\mathscr{M}}(\mathbf{C})$ :

$$
\sum_{n=1}^{\infty} \frac{1}{n^{2}}(z-n)^{1 / n}
$$

(values of the $n$th roots can be chosen arbitrarily). In this example the periods of the germs considered depend on the loop while the set of branching points is discrete. But the presence of "exceptional set" in the definitions implies that there are more exotic germs in $\mathscr{P}$, e.g.,

$$
\sum_{n=1}^{\infty} \frac{1}{n^{2}}\left(z-\frac{n+1}{n}\right)^{1 / 2} \in \mathscr{H}_{0} .
$$

For the "periodically multivalued" function germs just introduced, Theorem 1 can be extended as follows.

Theorem 2. Put $S_{0}=\mathscr{H}_{\zeta} \cap \mathscr{P}$. For $n \in \mathbf{N}$ put $B_{n}=$ the multiplicative subgroup of $\mathscr{H}_{\zeta}^{*}$ generated by $\bigcup\left\{\right.$ the set of all branches of $f^{g} \mid f \in \mathscr{H}_{\zeta}^{*} \cap \mathscr{P}$ and $g \in$ $\left.\mathscr{H}_{\zeta} \cap S_{n-1}\right\}$, and $S_{n}=$ the subring of $\mathscr{H}_{\zeta}$ generated by $S_{n-1} \cup B_{n}$.

Let $h_{0}, \ldots, h_{m} \in \bigcup_{n=1}^{\infty} B_{n}$. If $\left\{h_{j}\right\}_{j}$ are linearly dependent over $\mathscr{M}_{\zeta} \cap \mathscr{P}$ then there exist $j \neq k$ such that $h_{j} / h_{k} \in \mathscr{M}_{\zeta} \cap \mathscr{P}$.

The next subsection contains a van den Dries type theorem for exponential rings, which will be used as a lemma later in this section. Theorems 1 and 2 will be proved in $\S 4.3$ together with some other results.

Germs of class $\mathscr{P}$ resemble algebroid functions very much. What can be done for larger classes of "few-valued" functions, e.g., for

$$
\sum_{n=1}^{\infty} c_{n}(z-n)^{r_{n}}
$$

where $r_{n} \in \mathbf{R}, c_{n}$ are constants, and $c_{n} \rightarrow 0$ sufficiently rapidly? (If at least one $r_{n}$ is irrational with $c_{n} \neq 0$ then such germs are not in $\mathscr{P}$.) These germs are few-valued in the following sense.

Definition. Let $g \in \mathscr{M}_{\zeta}$. We shall write $g \in \mathscr{A}(U)$ or, shorter, $g \in \mathscr{A}$, if there exists an exceptional set $K \subset U, \zeta \notin K$, such that for every 1-periodic holomorphic mapping $\mu$ of the strip $A=\{w \in \mathbf{C} \mid-1<\operatorname{im} w<1\}$ into $U-K$ with $\mu(0)=\zeta$ the germ $g \circ \mu$ continues analytically to a holomorphic almost periodic function on $A$.

Some words in the above definition have to be explained. We consider the space $\mathscr{B}$ of holomorphic functions on $A$ bounded on every strip $A_{\varepsilon}=$ 
$\{w \in \mathbf{C} \mid-1+\varepsilon<\operatorname{im} w<1-\varepsilon\}$ for $\varepsilon>0$. The topology on $\mathscr{B}$ is given by the family of norms $\|h\|_{\varepsilon}=\sup _{w^{\prime} \in A_{\varepsilon}}|h(w)|$. In the above definition, a function $f$ is called almost periodic on $A$ if $f \in \mathscr{B}$ and the set of translates $\left\{f_{x}\right\}_{x}, f_{x}(w)=f(w+x)$ for $w \in \mathbf{R}$, is precompact (= totally bounded) in the topology of $\mathscr{B}$.

The class $\mathscr{A}$ is much larger than $\mathscr{P}$. For example, let $K$ be the Cantor set in $[0,1]$ and $\left\{t_{n}\right\}_{n}$ be an enumeration of the rational points of $K$. Then for any $\left\{r_{n}\right\}_{n} \subset \mathbf{R}$ with either at least one $r_{n} \notin \mathbf{Q}$ or $\left\{r_{n}\right\}_{n} \subset \mathbf{Q}$ having no common denominator

$$
\sum_{n=1}^{\infty} c_{n}\left(z-t_{n}\right)^{r_{n}} \in \mathscr{A}-\mathscr{P},
$$

where $\left\{c_{n}\right\}_{n}$ are nonzero constants and $c_{n} \rightarrow 0$ sufficiently rapidly. In particular,

$$
\sum_{n=1}^{\infty} \frac{1}{n^{2}}\left(z-t_{n}\right)^{1 / \sqrt{n}} \text { and } \sum_{n=1}^{\infty} \frac{1}{n^{2}}\left(z-t_{n}\right)^{1 / 2^{n}}
$$

are "almost periodically multivalued" but not "periodically multivalued". Theorems 1 and 2 have an analogue for $\mathscr{A}$.

Theorem 3. Put $S_{0}=\mathscr{H}_{\zeta} \cap \mathscr{A}$. For $n \in \mathbf{N}$ put $B_{n}=$ the multiplicative subgroup of $\mathscr{H}_{\zeta}^{*}$ generated by $\bigcup\left\{\right.$ the set of all branches of $f^{g} \mid f \in \mathscr{H}_{\zeta}^{*} \cap \mathscr{A}$ and $g \in \mathscr{H}_{\zeta} \cap$ $\left.S_{n-1}\right\}$, and $S_{n}=$ the subring of $\mathscr{H}_{\zeta}$ generated by $S_{n-1} \cup B_{n}$.

Let $h_{0}, \ldots, h_{m} \in \cup_{n=1}^{\infty} B_{n}$. If $\left\{h_{j}\right\}_{j}$ are linearly dependent over $\mathscr{M}_{\zeta} \cap \mathscr{A}$ then there exist $j \neq k$ such that $h_{j} / h_{k} \in \mathscr{M}_{\zeta} \cap \mathscr{A}$.

For lack of time we present not the proof of this theorem, but only some hints for it.

4.2. Adjoining an integral to an exponential field. Let $\mathscr{S}$ be a commutative differential field containing at least one nonconstant and let exp: $(\mathscr{S},+) \rightarrow$ $(\mathscr{S}-\{0\}, \cdot)$ be a group homomorphism such that $(\exp (a))^{\prime}=a^{\prime} \cdot \exp (a)$ for any $a \in \mathscr{S}$. Then exp cannot be identically 1 and by a remark of K. Manders (see the very beginning of [8]) the characteristic of $\mathscr{S}$ is 0 . Below we frequently write $e^{a}$ for $\exp (a)$.

Let $\mathscr{F}$ be a subfield of $\mathscr{S}$ containing all constants and closed under exponentiation and differentiation. Let $w \in \mathscr{S}$ be such that $w^{\prime}=1$ and $w \notin \mathscr{F}$. Then $w$ is transcendental over $\mathscr{F}$, and one can refer to the subring of $\mathscr{S}$ generated by $\mathscr{F}$ and $w$ as $\mathscr{F}[w]$ and to the subfield of $\mathscr{S}$ generated by $\mathscr{F}$ and $w$ as $\mathscr{F}(w)$. We consider in this subsection the exponential subring of $\mathscr{S}$ generated by $\mathscr{F}$ and $w$, and prove a van den Dries type result for it; see [8]. The examples we have in mind are of the form $\mathscr{S}=\mathscr{E}$ (a domain in $\mathbf{C}$ ) with $\mathscr{F}$ an exponential subfield of $\mathscr{S}$ not containing the identity function (see more details below). The condition $w^{\prime}=1$ was taken mostly for convenience. It is less restrictive than it may seem to be; if $u^{\prime} \in \mathscr{F}$ and $u \notin \mathscr{F}$ then one can take $u$ instead of $w$ and redefine the differentiation by putting (the new derivative) $=($ the old derivative $) / u^{\prime}$. Thus the title of this subsection is justified: what we prove below for $w^{\prime}=1$ will be applicable to any integral adjoined to $\mathscr{F}$. (For more information on differential fields see [24].)

Remark 1. Let $p \in \mathscr{F}[w]$. If $e^{p} \in \mathscr{F}(w)$ then $p=p_{1} w+p_{0}$ with $p_{1}=$ const and $p_{0} \in \mathscr{F}$. 
Proof. Let $p=p_{n} w^{n}+\cdots+p_{1} w+p_{0}$ with $p_{j} \in \mathscr{F} \quad(n \geq j \geq 0), p_{n} \neq 0$, and $e^{p}=q / r$, where $q, r \in \mathscr{F}[w]$. Then $\left(q^{\prime} r-q r^{\prime}\right) / r^{2}=(q / r)^{\prime}=\left(e^{p}\right)^{\prime}=$ $p^{\prime} \cdot e^{p}=p^{\prime} q / r$ and $p^{\prime} q r=q^{\prime} r-q r^{\prime}$. Since $\operatorname{deg}\left(p^{\prime} q r\right)=\operatorname{deg}\left(p^{\prime}\right)+\operatorname{deg}(q)+\operatorname{deg}(r)$ and $\operatorname{deg}\left(q^{\prime} r-q r^{\prime}\right) \leq \operatorname{deg}(q)+\operatorname{deg}(r)$ the latter identity implies $\operatorname{deg}\left(p^{\prime}\right) \leq 0$. Consider the case of $p_{n} \neq$ const. Then $p^{\prime}=p_{n}^{\prime} w^{n}+$ (summands of lower degree) and $\operatorname{deg}\left(p^{\prime}\right)=n>0$. Consider the case of $p_{n}=$ const. Then $p^{\prime}=$ $\left(n p_{n}+p_{n-1}^{\prime}\right) w^{n-1}+$ (summands of lower degree). If $n p_{n}+p_{n-1}^{\prime}=0$ then $\left(-p_{n-1} /\left(n p_{n}\right)\right)^{\prime}=1$ and $w=-p_{n-1} /\left(n p_{n}\right)+$ const, contradicting $w \notin \mathscr{F}$. Thus $n p_{n}+p_{n-1}^{\prime} \neq 0$ and $\operatorname{deg}\left(p^{\prime}\right)=n-1$. Hence $n \leq 1$ by $\operatorname{deg}\left(p^{\prime}\right) \leq 0$ and so $n=1$ and $p_{n}=$ const.

Examples. The following show that the situation $e^{p w} \in \mathscr{F}$ may occur. Let $\varepsilon \in \mathbf{R}_{+}$, and let $\mathscr{S}=\mathscr{E}(\{w \in \mathbf{C} \mid-\varepsilon<\operatorname{im} w<\varepsilon\})$.

(i) Put $\mathscr{F}=\{f \in \mathscr{S} \mid f$ is periodic with period 1$\}$; then $e^{p w} \in \mathscr{F}$ for $p \in 2 \pi i \mathbf{Z}$ and $e^{p w} \notin \mathscr{F}$ for all other $p$.

(ii) Put $\mathscr{F}=\{f \in \mathscr{S} \mid f$ is periodic with an integer period $\}$; then $e^{p w} \in \mathscr{F}$ for $p \in 2 \pi i \mathbf{Q}$ and $e^{p w} \notin \mathscr{F}$ for other $p$.

(iii) Put $\mathscr{F}=$ the exponential field generated by $\{f \in \mathscr{S} \mid f$ is holomorphic and almost periodic in $w\}$; then $e^{p w} \in \mathscr{F}$ for $p \in i \mathbf{R}$ and $e^{p w} \notin \mathscr{F}$ for re $p \neq 0$. Indeed, if a function $f$ can be built up of almost periodic functions then there exist $w$ and sequences $\left\{x_{n}\right\}_{n}$ and $\left\{y_{n}\right\}_{n}$ of reals such that $x_{n} \rightarrow \infty$, $y_{n} \rightarrow \infty$, and $\left\{f\left(w-p x_{n}\right)\right\}_{n},\left\{f\left(w+p y_{n}\right)\right\}_{n}$ are bounded.

(iv) Put $\mathscr{F}=$ the exponential subfield of $\mathscr{S}$ generated by $\left\{e^{c w} \mid c \in \mathbf{C}\right\}$.

The fact that $w \notin \mathscr{F}$ in (iv) follows from the results of $\S 2$. In cases (ii) and (iii) the additive group $\left\{p \mid e^{p w} \in \mathscr{F}\right\}$ (being a $\mathbf{Q}$-linear subspace of $\mathbf{C}$ ) can be complemented in the additive group $\mathbf{C}$ of all constants of $\mathscr{S}$. In case (iv) the complement of $\left\{p \mid e^{p w} \in \mathscr{F}\right\}$ in $\mathbf{C}$ is trivial: $\{0\}$.

Whenever the additive group $\left\{p \mid e^{p w} \in \mathscr{F}\right\}$ can be complemented in the additive group of all constants of $\mathscr{S}$, the exponential subring $\mathscr{R}$ of $\mathscr{S}$ generated by $\mathscr{F}$ and $w$ can be described as follows. Fix an additive subgroup $K$ of $\mathscr{F}$ complementing $\left\{p \mid e^{w p} \in \mathscr{F}\right\}$. Then $\mathscr{R}=\bigcup_{n=0}^{\infty} R_{n}$, where $\left\{R_{n}\right\}_{n}$ is an increasing sequence of differential subrings of $\mathscr{S}$ defined by the initial conditions

$$
R_{0}=\mathscr{F}[w], \quad A_{0}=\left\{e^{q w} \mid q \in \mathscr{F}[w] \text { and } q(0) \in K\right\},
$$

and induction,

$$
\begin{aligned}
R_{n+1}= & \text { the ring generated by } R_{n} \cup A_{n}, \\
A_{n+1}= & \text { the multiplicative group generated by } \\
& \left\{e^{r a} \mid r \in R_{n} \text { and } a \in A_{n}, a \neq 1\right\} .
\end{aligned}
$$

Note that $a^{\prime} / a \in R_{n}$ for each $a \in A_{n}$.

Proposition 2. Each $R_{n+1}$ is $R_{n}\left[A_{n}\right]$, i.e., for each $n$ the elements of $A_{n}$ are linearly independent over $R_{n}$.

As one will see from the proof, this result can be generalized to the "severalvariable" case.

Theorem 3. Let $\widetilde{\mathscr{S}}$ be a commutative exponential field with a number of differentiations $\left\{D_{j}\right\}_{j}$ such that $D_{j}\left(e^{a}\right)=D_{j}(a) \cdot e^{a}$ for each $D_{j}$ and each $a \in \tilde{\mathscr{S}}$. Let 
$\widetilde{\mathscr{F}}$ be an exponential subfield of $\widetilde{\mathscr{S}}$ closed under all $\left\{D_{j}\right\}_{j}$ and $\left\{w_{j}\right\}_{j} \subset \widetilde{\mathscr{S}}-\widetilde{\mathscr{F}}$ be such that $D_{i}\left(w_{j}\right)=\delta_{i j}$, where $\delta_{i j}$ is the Kronecker symbol. Suppose that each additive group $\left\{p \mid e^{p w_{j}} \in \widetilde{\mathscr{F}}\right\}$ has a complement $K_{j}$ in the additive group of $\widetilde{\mathscr{F}}$. Then the exponential subring $\widetilde{\mathscr{R}}$ of $\widetilde{\mathscr{S}}$ generated by $\widetilde{\mathscr{F}}$ and $\left\{w_{j}\right\}_{j}$ is $\bigcup_{n=0}^{\infty} \widetilde{R}_{n}$, where $\left\{\widetilde{R}_{n}\right\}_{n}$ is an increasing sequence of subrings of $\widetilde{\mathscr{S}}$ closed under all $D_{j}$ defined by the initial conditions

$$
\begin{gathered}
\widetilde{R}_{0}=\widetilde{\mathscr{F}}\left[\left\{w_{j}\right\}_{j}\right], \\
\widetilde{A_{0}}=\left\{\exp \left(\sum_{j} q_{j} w_{j}\right) \mid q_{j} \in \widetilde{\mathscr{F}}\left[\left\{w_{j}\right\}_{j}\right] \text { and } q_{j}(0) \in K_{j} \text { for all } j\right\},
\end{gathered}
$$

and induction,

$$
\begin{aligned}
\widetilde{R}_{n+1}= & \text { the ring generated by } \widetilde{R}_{n} \cup \widetilde{A}_{n}, \\
\widetilde{A}_{n+1}= & \text { the multiplicative group generated by } \\
& \left\{e^{r a} \mid r \in \widetilde{R}_{n} \text { and } a \in \widetilde{A}_{n}, a \neq 1\right\},
\end{aligned}
$$

and each $\widetilde{R}_{n+1}$ is $\widetilde{R}_{n}\left[\widetilde{A}_{n}\right]$, i.e., the elements of $\widetilde{A}_{n}$ are linearly independent over $\widetilde{R}_{n}$.

This latter theorem is an exact analogue of the van den Dries theorem on free exponential ring over an exponential ring in [8]; in the terms of this section, L. van den Dries was assuming that " $\widetilde{F}$ " contained only constants. Below we prove Proposition 2 only (the generalization is obvious). Proposition 2 remains valid for $K$ any additive subgroup of $\mathscr{F}$ such that $e^{p w} \notin \mathscr{F}$ for $p \in K-\{0\}$. However $\bigcup_{n=0}^{\infty} R_{n}$ is not the exponential ring generated by $\mathscr{F}$ and $w$ unless $K$ is a complement of $\left\{p \mid e^{p w} \in \mathscr{F}\right\}$ in the additive group of $\mathscr{F}$.

Proof of Proposition 2. Proceed by induction on $w$. For each $n$ the proof will be done in the following three steps:

(in) $A_{n} \cap R_{n}=\{1\}$,

(ii $i_{n} A_{n} \cap$ (the field of quotients of $R_{n}$ ) $=\{1\}$,

$\left(\mathrm{iii}_{n}\right)$ the elements of $A_{n}$ are linearly independent over $R_{n}$,

and we will make use of the van den Dries index ind (described below in the induction step). The statement $\left(\mathrm{ii}_{n}\right)$ is equivalent to "any two elements of $A_{n}$ are linearly independent over $R_{n}$ ".

Induction base. $\left(\mathrm{i}_{0}\right)$ and $\left(\mathrm{ii}_{0}\right)$ are contained in Remark 1.

(iii $)$ Suppose the contrary. Let $a_{1}, \ldots, a_{k}$ be distinct elements of $A_{0}$ linearly dependent over $\mathscr{F}(w)$. Since $A_{0}$ is a multiplicative group one can assume without loss of generality that 1 is among $\left\{a_{j}\right\}_{j}$; let $a_{k}=1$. Then $f_{1} a_{1}+\cdots+f_{k-1} a_{k-1}=1$ for some $f_{1}, \ldots, f_{k-1} \in \mathscr{F}(w)$ with not all of $\left\{f_{j}\right\}_{j}$ equal to 0 . Take the minimal $l$ for which there are distinct $a_{1}, \ldots, a_{l} \in$ $A_{0}-\{1\}$ and $f_{1}, \ldots, f_{l} \in \mathscr{F}(w)-\{0\}$ such that $f_{1} a_{1}+\cdots+f_{l} a_{l} \in \mathscr{F}(w)$. Then $l>1$ by $\left(\mathrm{ii}_{0}\right)$. Denote $g=f_{1} a_{1}+\cdots+f_{l} a_{l}$. Then $g \neq 0$, for otherwise $f_{1}\left(a_{1} / a_{l}\right)+\cdots+f_{l-1}\left(a_{l-1} / a_{l}\right)=-f_{l}$, contradicting the choice of $l$. Without loss of generality one can assume that $g=1$. Let $a_{j}=e^{w p_{j}}$ for each $j$. Then by differentiation $\sum_{j=1}^{l}\left(f_{j}^{\prime}+f_{j} p_{j}+f_{j} p_{j}^{\prime} w\right) a_{j}=0$ with $f_{j}^{\prime}+f_{j} p_{j}+f_{j} p_{j}^{\prime} w=$ 
$\left(f_{j} a_{j}\right)^{\prime} / a_{j} \neq 0$, since $\left(f_{j} a_{j}\right)^{\prime}=0$ would imply $f_{j} a_{j}=$ const and $a_{j} \in \mathscr{F}(w)$, contradicting Remark 1 . This contradicts the choice of $l$.

Induction step. Assume (iii $\left.{ }_{j}\right)$ for $j=0, \ldots, n$. Following [8] define an ordinal-valued function ind on $R_{n}$ as follows. Put ind $(0)=0$ and $\operatorname{ind}(f)=$ $\operatorname{deg}(f)+1$ for $f \in \mathscr{F}[w]-\{0\}$. Suppose that ind is already defined on $R_{n}$, and consider $f \in R_{n+1}-R_{n}$. Let $f=g_{1} a_{1}+\cdots+g_{m} a_{m}+g$, where $g \in R_{n}$, $g_{1}, \ldots, g_{m} \in R_{n}-\{0\}$, and $a_{1}, \ldots, a_{m}$ are distinct elements of $A_{n}-\{1\}$. By (iii ${ }_{n}$ ) this representation of $f$ is unique. Put ind $(f)=\omega^{n} \cdot m+\operatorname{ind}(g)$. Note that ind $(h)<\omega^{k}$ whenever $h \in R_{k}$.

Two remarks on ind follow. Let $h \in R_{n+1}-\{0\}$ and ind $(h)=\sum_{k=n}^{0} \omega^{k} \cdot m_{k}$, where $m_{0}, m_{1}, \ldots, m_{n} \in \mathbf{N}_{0}$.

1st. If $m_{0} \neq 0$ then there exists an $f \in \mathscr{F}$ such that ind $\left((f h)^{\prime}\right)<\operatorname{ind}(h)$ and $(f h)^{\prime} \neq 0$. Indeed, $m_{0}$ corresponds to the $\mathscr{F}[w]$ part of $h$; let us present the $\mathscr{F}[w]$ part of $h$ in the form $h_{m_{0}} w^{m_{0}}+\cdots+h_{0}$, and take $f=1 / h_{m_{0}}$.

$2 n d$. Let $k=\min \left\{j \mid m_{j}>0\right\}, k>0$. Then there exists an $a \in A_{k}$ such that ind $(a h)<\operatorname{ind}(h)$. Indeed, consider the $R_{k}$ part of $h$; let us present it in the form $h_{1} a_{1}+\cdots+h_{l} a_{l}$, where $a_{1}, \ldots, a_{l}$ are distinct elements of $A_{k}$ and $h_{1}, \ldots, h_{l} \in R_{k-1}-\{0\}$, and take $a=1 / a_{l}$.

Further, $A_{n+1}$ is a torsion-free Abelian group. If $A_{n+1}$ contained an element of a finite order then for some $p_{1}, \ldots, p_{k} \in R_{n}-\{0\}$ and distinct $a_{1}, \ldots, a_{k} \in$ $A_{n}-\{1\}$ there would be $\left(\exp \left(p_{1} a_{1}+\cdots+p_{k} a_{k}\right)\right)^{m}=\exp \left(m p_{1} a_{1}+\cdots+m p_{k} a_{k}\right)=1$ whence $m p_{1} a_{1}+\cdots+m p_{k} a_{k}=$ const for some $m \in \mathbf{N}$. This is impossible by $\left(\mathrm{iii}_{n}\right)$.

$\left(\mathrm{i}_{n+1}\right)$ Suppose the contrary. Let $a_{1}, \ldots, a_{k}$ be distinct elements of $A_{n}-$ $\{1\}, p_{1}, \ldots, p_{k} \in R_{n}-\{0\}$, and $\exp \left(p_{1} a_{1}+\cdots+p_{k} a_{k}\right)=f \in R_{n+1}$. Let $f=q_{1} b_{1}+\cdots+q_{l} b_{l}+r$, where $b_{1}, \ldots, b_{l}$ are distinct elements of $A_{n}$ $\{1\}, q_{1}, \ldots, q_{l} \in R_{n}-\{0\}, r \in R_{n}$. Then $f^{\prime}=\tilde{q}_{1} b_{1}+\cdots+\tilde{q}_{l} b_{l}+r^{\prime}$, where $\tilde{q}_{1}, \ldots, \tilde{q}_{l} \in R_{n}-\{0\}, \tilde{q}_{j}=q_{j}^{\prime}+\left(b_{j}^{\prime} / b_{j}\right)$, and $r^{\prime} \in R_{n}$. On the other hand, $f^{\prime}=\left(\exp \left(p_{1} a_{1}+\cdots+p_{k} a_{k}\right)\right)^{\prime}=\left(p_{1} a_{1}+\cdots+p_{k} a_{k}\right)^{\prime} f=\left(\tilde{p}_{1} a_{1}+\cdots+\tilde{p}_{k} a_{k}\right) f$, where $\tilde{p}_{1}, \ldots, \tilde{p}_{k} \in R_{n}-\{0\}$. (By $\left(\mathrm{i}_{n}\right)$ each $p_{j} a_{j} \neq$ const and so $\left(p_{j}^{\prime}+a_{j}^{\prime} / a_{j}\right) a_{j} \neq 0$, $\tilde{p}_{j}=p_{j}^{\prime}+a_{j}^{\prime} / a_{j} \neq 0$.) Thus $\left(\tilde{p}_{1} a_{1}+\cdots+\tilde{p}_{k} a_{k}\right) \cdot\left(q_{1} b_{1}+\cdots+q_{l} b_{l}+r\right)=$ $\tilde{q}_{1} b_{1}+\cdots+\tilde{q}_{l} b_{l}+r^{\prime}$. The multiplication in the left-hand side yields $\sum_{\mu} s_{\mu} c_{\mu}=$ $\sum_{j=1}^{l} \tilde{q}_{j} b_{j}+r^{\prime}$, where each $c_{\mu} \in A_{n}$ is the product of an element of $\left\{a_{i}\right\}_{i=1}^{k}$ be an element of $\{1\} \cup\left\{b_{j}\right\}_{j=1}^{l}$ and $\left\{s_{\mu}\right\}_{\mu}$ are the corresponding coefficients. Since $A_{n}$ is an Abelian torsion-free group, it can be linearly ordered (see [4]), and one can assume that $a_{1}>\cdots>a_{k}$. Let $b^{*}$ be the maximum of $\left\{b_{j}\right\}_{j=1}^{l}$ if $r=0$ and the maximum of $\{1\} \cup\left\{b_{j}\right\}_{j=1}^{l}$ if $r \neq 0$. Similarly, let $b_{*}$ be the minimum of $\left\{b_{j}\right\}_{j=1}^{l}$ if $r=0$ and the minimum of $\{1\} \cup\left\{b_{j}\right\}_{j=1}^{l}$ if $r \neq 0$. Then at least one of $c^{*}=a_{1} b^{*}$ and $c_{*}=a_{k} b_{*}$ occurs with a nonzero coefficient in $\sum_{\mu} s_{\mu} c_{\mu}$ without occurring in $\sum_{j=1}^{l} \tilde{q}_{j} b_{j}+r^{\prime} \cdot 1$. The latter contradicts (iii ${ }_{n}$ ).

$\left(\mathrm{ii}_{n+1}\right)$ Suppose the contrary. Let $a \in A_{n+1}-\{1\}, f, g \in R_{n+1}-\{0\}$, and $f a=g$. Denote $\lambda=\operatorname{ind}(g), \lambda>0$. Without loss of generality one can assume that $p a \neq q$ whenever $p, q \in R_{n+1}-\{0\}$ and $\operatorname{ind}(q)<\lambda$. If $\lambda=1$ then $g \in \mathscr{F}$ and $(1 / a)=f / g \in R_{n+1}$ contradicting $\left(i_{n+1}\right)$. Let $\lambda>1$. Then either there exists a $p \in \mathscr{F}$ such that ind $\left((g p)^{\prime}\right)<\operatorname{ind}(g)$ and $(g p)^{\prime} \neq 0$ with $\left((f p)^{\prime}+\left(a^{\prime} / a\right)\right) a=(g p)^{\prime}$, contradicting the choice of $\lambda$, or there exists a 
$b \in A_{k}$ for some $k \leq n$ such that $\operatorname{ind}(g b)<\operatorname{ind}(g)$ with $(f b) a=g b$, again contradicting the choice of $\lambda$.

$\left(\mathrm{iii}_{n+1}\right)$ Suppose the contrary. Take the minimal $l$ for which there exist distinct $a_{1}, \ldots, a_{l} \in A_{n+1}-\{1\}$ and $f_{1}, \ldots, f_{l} \in$ (the field of quotients of $\left.R_{n+1}-\{0\}\right)$ such that $f_{1} a_{1}+\cdots+f_{l} a_{l} \in R_{n}$. Then $l>1$ by $\left(\mathrm{ii}_{n+1}\right)$. Denote $q=f_{1} a_{1}+\cdots+f_{l} a_{l}$. Then $g \neq 0$, for otherwise there would be $f_{1}\left(a_{1} / a_{l}\right)+$ $\cdots+f_{l-1}\left(a_{l-1} / a_{l}\right)=-f_{l}$, contradicting the minimality of $l$. Without loss of generality one can assume that $g=1$. By differentiating

$$
\sum_{j=1}^{l}\left(f_{j}^{\prime}+f_{j}\left(a_{j}^{\prime} / a_{j}\right)\right) a_{j}=0
$$

and hence

$$
\sum_{j=1}^{l-1}\left(f_{j}^{\prime}+f_{j}\left(a_{j}^{\prime} / a_{j}\right)\right)\left(a_{j} / a_{l}\right)=-\left(f_{l}^{\prime}+f_{l}\left(a_{l}^{\prime} / a_{l}\right)\right) .
$$

Each

$$
f_{j}^{\prime}+f_{j}\left(a_{j}^{\prime} / a_{j}\right)=\left(f_{j} a_{j}\right)^{\prime} / a_{j} \neq 0
$$

for otherwise there would be $f_{j} a_{j}=$ const with $a_{j} \in$ (the field of quotients of $\left.R_{n+1}\right)$, contradicting $\left(\mathrm{ii}_{n+1}\right)$. The above identity for $\left\{a_{j}\right\}_{j=1}^{l-1}$ contradicts the minimality of $l$.

4.3. Few-valued functions as bases for exponentiation. As was already mentioned, in order to have precise results one has to present all germs considered in a certain form. The common way to keep track of how some entity was constructed is the "proof-theoretic tree". However the logicians' proof-theoretic "trees" are not exactly trees in graph theory terminology: when some entity is used more than once in further construction, several edges can have the same tail vertex. So, we consider finite acyclic ${ }^{14}$ oriented graphs (without multiple edges) with some complex-analytic gear attached to them.

Let $E$ be a set of directed edges on a set $T$ of vertices. For $\varepsilon \in E$ denote by $\varepsilon_{\bullet}$ the head of $\varepsilon$ and by $\varepsilon^{\bullet}$ the tail of $\varepsilon$ (all edges are directed downward). A vertex $t \in T$ will be called a source of $(T, E)$ if $t \neq \varepsilon_{\bullet}$ for all $\varepsilon \in E$, and a sink of $(T, E)$ if $t \neq \varepsilon^{\bullet}$ for all $\varepsilon \in E$.

Let $V$ be a connected complex-analytic manifold; fix a point $\xi \in V$. Let $\mathscr{Q}$ be a class of germs (of "few-valued" functions). We shall consider the cases of $\mathscr{Q}$ being $\mathscr{M}, \overline{\mathscr{M}}, \mathscr{P}, \mathscr{A}$, and so we assume below that $\mathscr{H} \xi \cap \mathscr{Q}$ is always an algebra over $\mathbf{C}$.

Definition. A $(V, \xi, \mathscr{Q})$-system is a quadruple $(T, E, b, c)$, where $(T, E)$ is a finite acyclic oriented graph and $b: E \rightarrow \mathscr{H}_{\xi}, c: T \rightarrow \mathscr{H}_{\xi}$ are such that $e^{b_{\varepsilon}} \in \mathscr{Q}$ for each $\varepsilon \in E$ and $c_{t} \in \mathscr{Q}$ for each $t \in T$.

A couple of $(V, \xi, \mathscr{Q})$-systems are:

\footnotetext{
${ }^{14} \mathrm{~A}$ sequence $\varepsilon_{1}, \ldots, \varepsilon_{m}$ of edges in an oriented graph is called a cycle if the head vertex of $\varepsilon_{m}$ is the tail vertex of $\varepsilon_{1}$ and for each $j, 1 \leq j<m$, the head vertex of $\varepsilon_{j}$ is the tail vertex of $\varepsilon_{j+1}$. An oriented graph is called acyclic if it contains no cycle.
} 


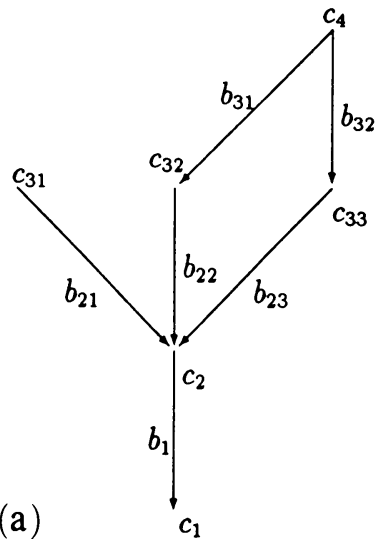

(a)

For a $(V, \xi, \mathscr{Q})$-system $(T, E, b, c)$ assign a germ $g_{t} \in \mathscr{H}_{\xi}$ to each $t \in T$ inductively as follows. If $t$ is a source of $(T, E)$ then put $g_{t}=c_{t}$. If $t$ is not a source and all $g_{\varepsilon^{\bullet}}$ for $\varepsilon_{\bullet}=t$ are already determined then put $g_{t}=$ $c_{t} \cdot \exp \left(\sum\left\{b_{\varepsilon} g_{\varepsilon} \cdot \mid \varepsilon_{\bullet}=t\right\}\right)$.

For the $(V, \xi, \mathscr{Q})$-systems shown above (denote by $\alpha$ the sink of (a) and by $\beta$ the sink of $(b))$

and

$$
g_{\alpha}=c_{1} \cdot\left(f_{1}\right)^{c_{2}}
$$

where each $f_{\text {? }}$ is $e^{b_{\text {? }}}$.

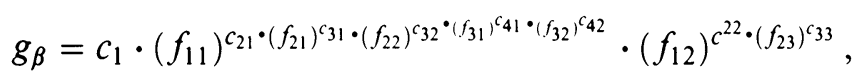

One can see immediately that these germs $g_{t}$ are exactly of the kind referred to in Theorems 1, 2, 3 of $\S 4$.1: for any finite family of germs $\left\{h_{j}\right\}_{j=0}^{m}$ mentioned in those theorems one can find a $(U, \zeta, \mathscr{Q})$-system

$$
(T, E, b, c) \text {, }
$$

where $\mathscr{Q}$ is $\overline{\mathscr{M}}$ or $\mathscr{P}$ or $\mathscr{A}$ respectively, with $\left\{t_{j}\right\}_{j=0}^{m}$ the sinks of $(T, E)$, such that $g_{t_{j}}=h_{j}$ for each $j$. For example,

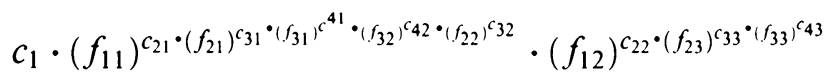

is presented by the following $(V, \xi, \mathscr{Q})$-system

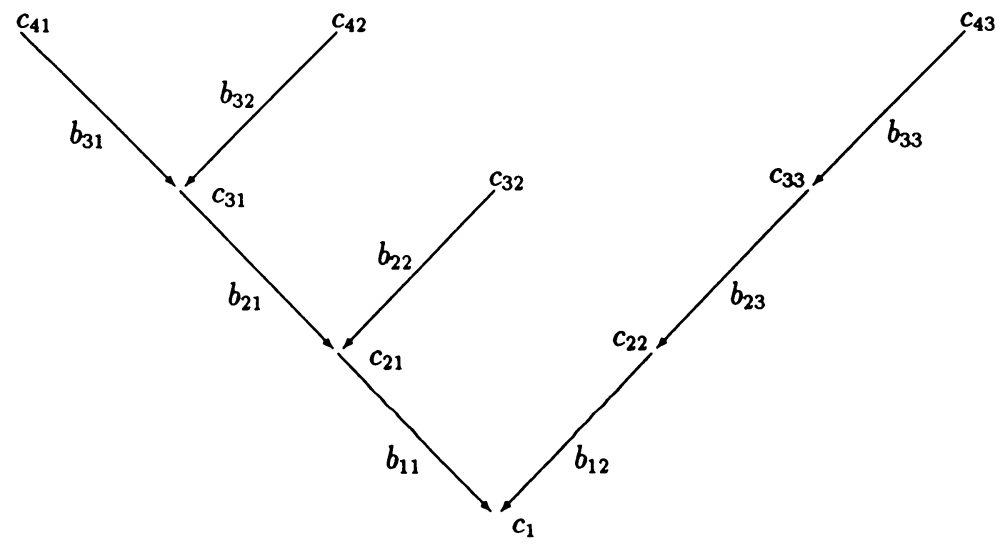

where each $b_{?}$ is $\log f_{?}$. 
The following is an analogue of the conditions " $\{1\} \cup\{$ the exponents $\}$ linearly independent over Q" in Examples 5, 6 of $\S 3.2$.

Definition (for $\mathscr{Q} \subset \mathscr{P}$ only). A $(V, \xi, \mathscr{Q})$-system $(T, E, b, c)$ will be called proper if the following conditions are satisfied:

(i) If $t$ is a source but not a sink then $c_{t}$ is not a rational constant.

(ii) For each $\varepsilon \in E$ either $b_{\varepsilon}=1$ or $b_{\varepsilon} \notin \mathscr{P}$, and only the latter holds when $\varepsilon^{\bullet}$ is a source.

(iii) The set $\{1\} \cup\left\{g_{\varepsilon^{\bullet}} \mid \varepsilon_{\bullet}=t\right\}$ is linearly independent over $\mathbf{Q}$ for all $t \in T$.

The meaning of these conditions can be seen from the following remark and its proof.

Remark 1. Let $\mathscr{Q}$ be $\overline{\mathscr{M}}$ or $\mathscr{P}$. Then for every family $\left\{h_{j}\right\}_{j=0}^{m} \subset \bigcup_{n=1}^{\infty} B_{n}$ (in the notation of Theorems 1 and 2 of $\S 4.1$ ) there exists a proper $(U, \zeta, \mathscr{Q}$ )system $(T, E, b, c)$ with $\left\{t_{j}\right\}_{j=0}^{m}$ the sinks of $(T, E)$ satisfying $g_{t_{j}}=h_{j}$ for each $j$.

Proof. For condition (i) above: if $f=e^{b_{\varepsilon}} \in \mathscr{Q}$ has to be raised to a rational constant power $q=c_{\varepsilon^{\bullet}} \cdot 1$ then $f^{q} \in \mathscr{Q}$, and one can change $c_{\varepsilon_{\bullet}}$ to $c_{\varepsilon_{\bullet}} f^{q}$ and drop the edge $\varepsilon$ from $(T, E)$. For condition (ii) above in the general case: if $b_{\varepsilon} \in \mathscr{Q}$ then one can change $c_{\varepsilon} \bullet$ to $c_{\varepsilon} \bullet b_{\varepsilon}$ and change $b_{\varepsilon}$ to 1 thereby making $(T, E, b, c)$ somewhat simpler. In the case of $t=\varepsilon^{\bullet}$ being a source in condition (ii) above: if $b_{\varepsilon} \in \mathscr{Q}$ then one can change $c_{\varepsilon_{\bullet}}$ to $c_{\varepsilon_{\bullet}} \cdot e^{c_{t} b_{\varepsilon}}$ and drop the edge $\varepsilon$ from $(T, E)$. For condition (iii) above: if $\left\{\varepsilon_{j}\right\}_{j=1}^{n}$ is an enumeration of $\left\{\varepsilon \mid \varepsilon_{\bullet}=t\right\}$, each $u_{j}=\left(\varepsilon_{j}\right)^{\bullet}$, and $g_{u_{n}}=r_{0}+\sum_{j=1}^{n-1} r_{j} g_{u_{j}}$ with some $r_{0}, \ldots, r_{n-1} \in \mathbf{Q}$ then one can change $c_{t}$ to $c_{t} \cdot \exp \left(r_{0} b_{\varepsilon_{n}}\right)$, change $b_{\varepsilon_{j}}$ to $b_{\varepsilon_{j}}+r_{j} b_{\varepsilon_{n}}$ for $1 \leq j<n$, and drop the edge $\varepsilon_{n}$ from $(T, E)$.

We consider first the case when all bases for exponentiation occurring have no singularities (zeros, poles or branching points) at all. A $(V, \xi, \mathscr{Q})$-system $(T, E, b, c)$ will be called nonsingular if all $\left\{b_{\varepsilon}\right\}_{\varepsilon \in E}$ and $\left\{c_{t}\right\}_{t \in T}$ can be analytically continued as holomorphic germs along every loop in $(V, \xi)$. The general case will be reduced (soon) to the nonsingular case.

The following result is a further analogue of Corollary 2 of $\S 1.3$ (which is an immediate corollary of the Hiromi-Ozawa lemma) and an improvement of the linear independence results of $\S 3.2$.

Theorem 2. Let $(T, E, b, c)$ be a proper nonsingular $(V, \xi, \mathscr{P})$-system, let $t, \ldots, u$ be the sinks of $(T, E)$ with none of them being a source of $(T, E)$. Then either $g_{t}+\cdots+g_{u}=0$ or $g_{t}+\cdots+g_{u}$ is nonperiodic with respect to some loop in $\pi_{1}(V, \xi)$.

Proof. Let $G=\left\{\sigma \in \pi_{1}(V, \xi) \mid\right.$ the analytic continuation along $\sigma$ leaves intact all $e^{b_{\varepsilon}}$ and $\left.c_{t}\right\}$. Then $G$ is a subgroup of $\pi_{1}(V, \xi)$ and for any $\lambda \in \pi_{1}(V, \xi)$ there exists an $n \in \mathbf{N}$ such that $\lambda^{n} \in G$. Consider

$$
\nu_{\varepsilon}=\frac{1}{2 \pi i}\left(\text { the increment of } b_{\varepsilon}\right. \text { along a loop), }
$$


for $\varepsilon \in E$. Then $\left\{\nu_{\varepsilon}\right\}_{\varepsilon}$ are homomorphisms $G \rightarrow \mathbf{Z}$. Let $\gamma \in G$ be such that for the subgroup $\Gamma \subset G$ generated by $\gamma$, for every subset $J \subset E$ the rank of $\left\{\nu_{\varepsilon}\right\}_{\varepsilon \in J}: G \rightarrow \mathbf{Z}^{J}$ coincides with the rank of $\left\{\left.\nu_{\varepsilon}\right|_{\Gamma}\right\}_{\varepsilon \in J}: \Gamma \rightarrow \mathbf{Z}^{J}$. We shall show that if $g_{t}+\cdots+g_{u}$ is not identically zero then it is nonperiodic with respect to $\gamma$.

Let $\lambda:\left(S^{1}, 1\right) \rightarrow(V, \xi)$ realize $\gamma$. In view of Lemma 1 of $\S 2.1$ one can assume that $\lambda$ is real analytic and one can assume without loss of generality

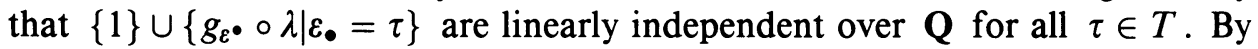
Lemma 2 of $\S 2.1$ one can continue $\lambda$ analytically to a holomorphic mapping $\mu$ : (the annulus $1-\alpha<|z|<1+\alpha) \rightarrow V$ for sufficiently small $\alpha \in \mathbf{R}_{+}$. Consider the strip $W=\{w \in \mathbf{C} \mid \log (1-\alpha)<2 \pi \operatorname{im} w<\log (1+\alpha)\}$. Then $\mu \circ e^{2 \pi i w}$ is a 1 -periodic holomorphic mapping $W \rightarrow V$. We are going to apply Proposition 2 of $\S 4.2$.

Put $\mathscr{S}=\mathscr{E}(W)$ and $\mathscr{F}=\{f \in \mathscr{S} \mid f$ is $n$-periodic for some $n \in \mathbf{N}\}$. Since $b_{\varepsilon} \notin \mathscr{P}$ for at least one $\varepsilon \in E$, one has $\gamma^{n} \neq 1$ for all $n \in \mathbf{N}$, and $\Gamma$ can be regarded as a copy of $\mathbf{Z}$. Then to each $\nu_{\varepsilon}$ there corresponds $m_{\varepsilon} \in \mathbf{Z}$ such that $\nu_{\varepsilon}(a)=m_{\varepsilon} a$, each $c_{\tau} \circ \mu \circ e^{2 \pi i w} \in \mathscr{F}$, and $b_{\varepsilon} \circ \mu \circ e^{2 \pi i w}=m_{\varepsilon} w+f_{\varepsilon}$, where $f_{e} \in \mathscr{F}$, and $f_{\varepsilon}=1$ whenever $m_{\varepsilon}=0$. Further,

$$
g_{\tau} \circ \mu \circ e^{2 \pi i w}=c_{\tau} \circ \mu \circ e^{2 \pi i w} \in \mathscr{F}-\{0\}
$$

for $\tau$ a source of $(T, E)$ and

$$
g_{\tau} \circ \mu \circ e^{2 \pi i w}=\left(c_{\tau} \circ \mu \circ e^{2 \pi i w}\right) \cdot \exp \left(\sum\left\{\left(m_{\varepsilon} w+f_{\varepsilon}\right) \cdot\left(g_{\varepsilon} \circ \mu \circ e^{2 \pi i w}\right) \mid \varepsilon_{\bullet}=\tau\right\}\right)
$$

for $\tau$ not a source of $(T, E)$. The former factor $c_{\tau} \circ \mu \circ e^{2 \pi i w}$ above belongs to $\mathscr{F}-\{0\}$; consider the latter factor:

$$
\exp \left(\sum\left\{\left(m_{\varepsilon} w+f_{\varepsilon}\right) \cdot\left(g_{\varepsilon} \bullet \mu \circ e^{2 \pi i w}\right) \mid \varepsilon_{\bullet}=\tau\right\}\right)
$$

It is equal to

$$
\begin{aligned}
& \exp \left(\sum\left\{f_{\varepsilon} \bullet\left(g_{\varepsilon^{\bullet}} \circ \mu \circ e^{2 \pi i w}\right) \mid \varepsilon_{\bullet}=\tau \text { and } \varepsilon^{\bullet} \text { is a source }\right\}\right) \\
& \times \exp \left(\sum\left\{m_{\varepsilon} w \cdot\left(g_{\varepsilon^{\bullet}} \circ \mu \circ e^{2 \pi i w}\right) \mid \varepsilon_{\bullet}=\tau\right\}\right)
\end{aligned}
$$

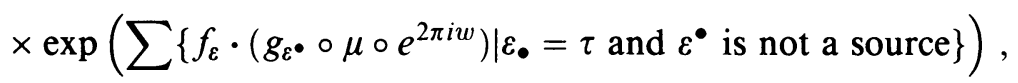

where the first factor belongs to $\mathscr{F}-\{0\}$; denote by $q_{\tau}$ the product of the latter two factors. We shall show that in the notation of Proposition 2 of $\S 4.2$ $q_{\tau}$ belongs to (the multiplicative group generated by $\left.\bigcup_{n} A_{n}\right)-\{1\}$ for each nonsource $\tau \in T$.

Proceed by induction on $\tau$. If $\varepsilon^{\bullet}$ is a source for each $\varepsilon$ with $\varepsilon_{\bullet}=\tau$ then $g_{\varepsilon^{\bullet}}=c_{\varepsilon^{\bullet}}$ and $q_{\tau}=\exp \left(\sum\left\{m_{\varepsilon} w \cdot\left(c_{\varepsilon} \bullet \mu \circ \circ e^{2 \pi i w}\right) \mid \varepsilon_{\bullet}=\tau\right\}\right)$, where all $m_{\varepsilon} \neq 0$. The latter function belongs to $A_{0}-\{1\}$ (since $\{1\} \cup\left\{c_{\varepsilon^{\bullet}} \mid \varepsilon_{\bullet}=\tau\right\}$ is linearly independent over $\mathbf{Q}$ on $\left.\lambda\left(S^{1}\right) \subset V\right)$. Consider the general case. Denote by $C_{k}$ the multiplicative group generated by $(\mathscr{F}-\{0\}) \cup\left(\bigcup_{l<k} A_{l}\right)$ for $k \in \mathbf{N}_{0}$. 
Let $N=\max \left\{k \mid g_{\varepsilon} \bullet \circ \mu \circ e^{2 \pi i w}=a p\right.$ for some $a \in A_{k}-\{1\}, p \in C_{k}$, and $\varepsilon \in E$ with $\left.\varepsilon_{0}=\tau\right\}$ (assume $\max \varnothing=-\infty$ ). If $N=-\infty$ then $\varepsilon^{\bullet}$ is a source for each $\varepsilon$ with $\varepsilon_{\bullet}=\tau$. For each $n \geq 0$ let $J_{n}=\left\{\varepsilon \mid \varepsilon_{\bullet}=\tau\right.$ and $g_{\varepsilon} \bullet \circ \circ e^{2 \pi i w}=a p$ for some $\left.a \in A_{n}-\{1\}, p \in C_{n}\right\}$. Let $g_{\varepsilon} \bullet \circ \circ \circ e^{2 \pi i w}=a_{\varepsilon} p_{\varepsilon}$ with $a_{\varepsilon} \in A_{n}-\{1\}, p_{\varepsilon} \in C_{n}$ for $\varepsilon \in J_{n}$. In view of Proposition 2 of $\S 4.2$ it suffices to show that $\sum\left\{\left(m_{\varepsilon} w+f_{\varepsilon}\right) \cdot p_{\varepsilon} a_{\varepsilon} \mid \varepsilon \in J_{n}\right\} \neq 0$. If $m_{\varepsilon} \neq 0$ for some $\varepsilon \in J_{n}$ then $\sum\left\{m_{\delta} p_{\delta} \mid \delta \in J_{n}, a_{\delta}=a_{\varepsilon}\right\} \neq 0$ (by the linear independence of $\left.\left\{p_{\varepsilon} a_{\varepsilon}\right\}_{\varepsilon \in J_{n}}\right)$ and this implies the result. If $m_{\varepsilon}=0$ for all $\varepsilon \in J_{n}$ then $f_{\varepsilon}=1$ for all $\varepsilon \in J_{n}, n \geq 0$, and $\sum\left\{p_{\varepsilon} a_{\varepsilon} \mid \varepsilon \in J_{n}\right\} \neq 0$ implies the result.

Corollary 3. Let $(T, E, b, c)$ be a proper $(V, \xi, \mathscr{P})$-system, let $t, \ldots, u$ be the sinks of $(T, E)$ with none of them a source of $(T, E)$. If $g_{t}+\cdots+g_{u}$ is not identically zero then $g_{t}+\cdots+g_{u} \notin \mathscr{P}$.

Proof. Take an exceptional set $K \subset V, \xi \notin K$, such that $(T, E, b, c)$ is a nonsingular $(V-K, \xi, \mathscr{P})$-system and apply Theorem 2 .

Now we are ready to prove Theorems 1 and 2 of $\S 4.1$. In the notation of those theorems, suppose the contrary: there exists an $m \in \mathbf{N}$ and $h_{0}, \ldots, h_{m} \in$ $\bigcup_{n=1}^{\infty} B_{n}$ such that $\left\{h_{j}\right\}_{j}$ are linearly dependent over $\mathscr{M}_{\zeta} \cap \overline{\mathscr{M}}(U)$ or over $\mathscr{M}_{\zeta} \cap \mathscr{P}(U)$ but pairwise noncollinear over $\mathscr{M}_{\zeta} \cap \overline{\mathscr{M}}(U)$ or (respectively) over $\mathscr{M}_{\zeta} \cap \mathscr{P}(U)$. Take the minimal such $m$. Without loss of generality one can assume that $h_{m}=1$, and then one can assume that $h_{0}+\cdots+h_{m-1}$ belongs to $\mathscr{M}_{\zeta} \cap \overline{\mathscr{M}}(U)-\{0\}$ or (respectively) to $\mathscr{M}_{\zeta} \cap \mathscr{P}(U)-\{0\}$ with $h_{j} \notin \overline{\mathscr{M}}(U)$ or respectively $h_{j} \notin \mathscr{P}(U)$ for $0 \leq j<m$. Let $(T, E, b, c)$ be a proper $(U, \zeta, \mathscr{P})$-system with $\left\{t_{j}\right\}_{j=0}^{m-1}$ the sinks of $(T, E)$ and such that $g_{t_{j}}=h_{j}$ for $0 \leq j<m$; see Remark 1 . Since $h_{j} \notin \overline{\mathscr{M}}(U)$ (respectively $h_{j} \notin \mathscr{P}(U)$ ) each $t_{j}$ is not a source of $(T, E)$. Since $g_{t_{0}}+\cdots+g_{t_{m-1}} \in \mathscr{P}$, Theorem 2 implies $g_{t_{0}}+\cdots+g_{t_{m-1}}=0$ and $h_{0}+\cdots+h_{m-1}=0$.

Now we shall hint at how Theorem 3 of $\S 4.1$ is proved.

Definition. A $(V, \xi, \mathscr{A})$-system $(T, E, b, c)$ will be called proper if the following conditions are satisfied.

(i) If $t$ is a source then $c_{t}$ is not a real constant.

(ii) For each $\varepsilon \in E$ either $b_{\varepsilon}=1$ or $b_{\varepsilon} \notin \mathscr{A}$, and only the latter holds when $\varepsilon^{\bullet}$ is a source.

(iii) The set $\{1\} \cup\left\{g_{\varepsilon^{\bullet}} \mid \varepsilon_{\bullet}=t\right\}$ is linearly independent over $\mathbf{R}$ for all $t \in T$.

This properness definition differs from the definition of properness of $(V, \xi, \mathscr{Q})$-systems for $\mathscr{Q}=\mathscr{M}, \overline{\mathscr{M}}, \mathscr{P}$ by the replacement of $\mathbf{Q}$ by $\mathbf{R}$. In analogy with Remark 1 above, there is

Remark 4. For every family $\left\{h_{j}\right\}_{j=0}^{m} \subset \bigcup_{n=1}^{\infty} B_{n}$ in the notation of Theorem 3 of $\S 4.1$ there exists a proper $(U, \zeta, \mathscr{A})$-system $(T, E, b, c)$ with $\left\{t_{j}\right\}_{j=0}^{m}$ the sinks of $(T, E)$ satisfying $g_{t_{j}}=h_{j}$ for each $j$.

The next result is an analogue of Theorem 2 and Corollary 3 (recall that $\mathscr{A}$ is the class of almost periodically multivalued functions).

Theorem 5. Let $(T, E, b, c)$ be a proper $(V, \xi, \mathscr{A})$-system, let $t, \ldots, u$ be the sinks of $(T, E)$ with none of them a source of $(T, E)$. Then either $g_{t}+$ $\cdots+g_{u}=0$ or $g_{t}+\cdots+g_{u} \notin \mathscr{A}$. 
For the lack of time we do not present the proof. The lemmas used are those of $\S 3.1$, the argument theorem for almost periodic functions (see [28]), and Proposition 2 of $\S 4.2$ as applied to example (iii) there.

Now, Theorem 3 of $\S 4.1$ can be derived from Theorem 5 in the same way as Theorems 1 and 2 of $\S 4.1$ were derived from Corollary 3.

4.4. An application to Tarski's high school algebra problem: proof of the HensonRubel conjecture. Recall that the Schanuel class $\Sigma=\left\{t \in L \mid\right.$ if $a^{b}$ occurs as a subterm of $t$ then $a$ contains no variables $\}$, and the Levitz class $\Lambda=\{t \in L \mid$ if $a^{b}$ occurs as a subterm of $t$ then either $a$ is a variable or $a$ contains no variables $\}$. Put $\mathscr{S}_{0}=\Sigma$ and $\mathscr{L}_{0}=\Lambda$. For each $n \in \mathbf{N}$ put $\mathscr{S}_{n}=$ the closure of $\mathscr{S}_{n-1} \cup\left\{a^{b} \mid a \in \Sigma, b \in \mathscr{S}_{n-1}\right\}$ under addition and multiplication in $L$, and $\mathscr{L}_{n}=$ the closure of $\mathscr{L}_{n-1} \cup\left\{a^{b} \mid a \in \Lambda, b \in \mathscr{L}_{n-1}\right\}$ under addition and multiplication in $L$. Then

$$
\begin{aligned}
& \Sigma=\mathscr{S}_{0} \subset \mathscr{S}_{1} \subset \mathscr{S}_{2} \subset \mathscr{S}_{3} \subset \cdots \\
& \Lambda=\mathscr{L}_{0} \subset \mathscr{L}_{1} \subset \mathscr{L}_{2} \subset \mathscr{L}_{3} \subset \cdots,
\end{aligned}
$$

and as in $\S 3.3$ one can see that not only all inclusions shown are strict, but even $\mathscr{L}_{n}-\mathscr{L}_{n-1}-\bigcup_{k=0}^{\infty} \mathscr{S}_{k} \neq \varnothing$ for each $n \in \mathbf{N}$.

The +-primes and the $(+, \cdot)$-primes of $\bar{\Sigma}, \bar{\Lambda}, \overline{\mathscr{S}_{1}}, \overline{\mathscr{L}_{1}}$ were described in $\S \S 1.4$ and 3.3. ${ }^{15}$ In this subsection we provide a similar description (as outlined in $\S 1.2)$ of the arithmetic structure of $\mathscr{S}_{n}$ and $\mathscr{L}_{n}$ for all $n$. This description will imply the Tarski conjecture for $\mathscr{L}=\bigcup_{n=0}^{\infty} \mathscr{L}_{n}=\left\{t \in L \mid\right.$ if $a^{b}$ occurs as a subterm of $t$ then $a \in \Lambda\}$ (this class is much larger than $\Pi=\left\{t \in L \mid\right.$ if $a^{b}$ occurs as a subterm of $t$ then $a$ is a polynomial $\}$ about which $\mathrm{C}$. W. Henson and L. A. Rubel asked in [19]).

Clearly, every +-prime in $\overline{\mathscr{S}_{n}}, n>0$, can be presented in the form

(a monic monomial)

$$
\begin{aligned}
& \times \prod_{i}(\text { a monomial, } \neq 1)_{i} \uparrow\left(\text { a }+ \text {-prime } \in \overline{\mathscr{S}_{n-1}}, \neq 1\right)_{i} \\
& \times \prod_{j}(\text { a function } \in \bar{\Sigma}-\{1\} \text { with content }=1)_{j} \\
& \uparrow\left(\text { a }+ \text {-prime } \in \overline{\mathscr{S}_{n-1}}, \neq 1\right)_{j},
\end{aligned}
$$

where $\prod_{i}$ or $\prod_{j}$ (or both) may be empty, in each of those products all exponents occurring are distinct.

Proposition 1. For every $n \in \mathbf{N}$ :

$\left(\mathrm{i}_{n}\right)$ Let $p, p^{\prime} \in \overline{\mathscr{S}}_{n}$ possess representations of the form $\left(\nabla_{n}\right)$. If $p / p^{\prime}=\mathrm{const}$ then $p=p^{\prime}$ and their $\left(\nabla_{n}\right)$ representations coincide up to a permutation of factors.

$\left(\mathrm{ii}_{n}\right)$ Let $\left\{f_{k}\right\}_{k} \subset \overline{\mathscr{S}_{n}}$ possess representations of the form $\left(\bigcirc_{n}\right)$. If $f_{l} \neq f_{m}$ for $l \neq m$ then $\left\{f_{k}\right\}_{k}$ is linearly independent over $\mathbf{R}$.

$\left(\mathrm{iii}_{n}\right)$ The $\$$-primes of $\overline{\mathscr{S}}_{n}$ remain $\$$-prime in $\overline{\mathscr{S}_{n}}$ for $\$ \in\{+, \cdot\}$.

${ }^{15}$ Recall that the $(+, \cdot)$-primes of $\bar{\Lambda}$ (and of $\bar{\Sigma}$ in particular) are algebraically independent, and the content of functions in $\bar{\Lambda}$ is taken with respect to the $(+, \cdot)$-primes of $\bar{\Lambda}$. 
An immediate consequence of $\left(\mathrm{ii}_{n}\right)$ is that the +-primes of $\overline{\mathscr{S}}_{n}$ are precisely the functions which can be presented in the form $\left(\nabla_{n}\right)$. Indeed, let $f$ have representation of the form $\left(\nabla_{n}\right)$. If $f=g+h$ and $g, h \in \overline{\mathscr{S}}_{n}$, then upon a decomposition of $g$ and $h$ into sums of functions of the form $\left(\nabla_{n}\right)$ one has $f=$ (a sum of several functions of the $\left(\nabla_{n}\right)$-form). But this identity is a nontrivial linear relation between functions in the $\left(\nabla_{n}\right)$-form, which is impossible by $\left(\mathrm{ii}_{n}\right)$.

Proof. Proceed by joint induction on $n$ together with the following auxiliary statement.

$\left(a_{n}\right)$ For every $f \in \overline{\mathscr{S}}_{n}$ possessing a representation of the form $\left(\nabla_{n}\right)$ there exists a proper $\left(\mathbf{C}^{m}, \overrightarrow{1}, \mathscr{H}\right)$-system $(T, E, b, c)$ (where $m=$ the number of variables involved, and $\overrightarrow{1} \rightarrow \mathbf{C}^{m}$ has all coordinates $\left.=1\right)$ with unique sink $t$ of $(T, E)$ such that $f=g_{t}, c_{t}$ is a +-prime of $\bar{\Sigma}$, and $c_{\tau}$ is a +-prime of $\bar{\Sigma}$ for each source $\tau$ of $(T, E)$.

The case $n=1$ is contained in $\$ 3.3$. Consider the induction step. In order to prove $\left(\mathrm{a}_{n+1}\right)$ and $\left(\mathrm{i}_{n+1}\right)$, for each $p, p^{\prime} \in \overline{\mathscr{S}}_{n+1}$ and possessing $\left(\nabla_{n+1}\right)$ representations, we shall construct a $\left(\mathbf{C}^{m}, \overrightarrow{1}, \mathscr{M}\right)$-system $(T, E, b, c)$ in a certain way.

Let $p=\mu \cdot \prod_{i}\left(\alpha_{i} \uparrow \beta_{i}\right) \cdot \prod_{j}\left(\gamma_{j} \uparrow \delta_{j}\right)$ and $p^{\prime}=\mu^{\prime} \cdot \prod_{i^{\prime}}\left(\alpha_{i^{\prime}}^{\prime} \uparrow \beta_{i^{\prime}}^{\prime}\right) \cdot \prod_{j^{\prime}}\left(\gamma_{j^{\prime}}^{\prime} \uparrow \delta_{j^{\prime}}^{\prime}\right)$ according to $\left(\nabla_{n+1}\right)$. Let $\left\{\eta_{k}\right\}_{k}$ be a list without repetitions of the elements of $\left\{\beta_{i}\right\}_{i} \cup\left\{\delta_{j}\right\}_{j}$, and $\left\{\eta_{k^{\prime}}^{\prime}\right\}_{k^{\prime}}$ be a list without repetitions of the elements of $\left\{\beta_{i^{\prime}}^{\prime}\right\}_{i^{\prime}} \cup\left\{\delta_{j^{\prime}}^{\prime}\right\}_{j^{\prime}}$. In the representation of $p$ unite $\left(\alpha_{i} \uparrow \beta_{i}\right)$ with $\left(\gamma_{j} \uparrow \delta_{j}\right)$ into $\left(\left(\alpha_{i} \beta_{j}\right) \uparrow \eta_{k}\right)$ whenever $\beta_{i}=\delta_{j}=\eta_{k}$, and do the same for $p^{\prime}$. Then

$$
p=\mu \cdot \prod_{k}\left(\zeta_{k} \uparrow \eta_{k}\right) \quad \text { and } \quad p^{\prime}=\mu^{\prime} \cdot \prod_{k^{\prime}}\left(\zeta_{k^{\prime}}^{\prime} \uparrow \eta_{k^{\prime}}^{\prime}\right)
$$

where

$$
\zeta_{k}=\xi_{k} \theta_{k}, \quad \xi_{k}=\left\{\begin{array}{ll}
\alpha_{i} & \text { if } \eta_{k}=\beta_{i}, \\
1 & \text { otherwise },
\end{array} \quad \theta_{k}= \begin{cases}\gamma_{j} & \text { if } \eta_{k}=\delta_{j}, \\
1 & \text { otherwise }\end{cases}\right.
$$

and the $\zeta_{k^{\prime}}^{\prime}$ are defined similarly, and in each of $\Pi_{k}$ and $\Pi_{k^{\prime}}$ all exponents occurring are distinct. Note for $\left(i_{n+1}\right)$ : to establish the coincidence of the $\left(\nabla_{n+1}\right)$ representations of $p$ and $p^{\prime}$ it suffices to establish the coincidence of their representations in $(*)$. Let $\left\{\rho_{l}\right\}_{l}$ be a list without repetitions of the elements of $\left\{\eta_{k}\right\}_{k} \cup\left\{\eta_{k^{\prime}}^{\prime}\right\}_{k^{\prime}}$. Put

$$
\psi_{l}=\frac{\text { if } \rho_{l}=\eta_{k} \text { then } \zeta_{k} \text { else } 1}{\text { if } \rho_{l}=\eta_{k^{\prime}}^{\prime} \text { then } \zeta_{k^{\prime}}^{\prime} \text { else } 1}
$$

for each $l$. Then

$$
p / p^{\prime}=\left(\mu / \mu^{\prime}\right) \cdot \prod_{l}\left(\psi_{l} \uparrow \rho_{l}\right)
$$

where $\left\{\rho_{l}\right\}_{l}$ are distinct +-primes of $\overline{\mathscr{S}_{n}}$ and each $\psi_{l}$ is a quotient of functions of the form (a monomial) $\cdot\left(\right.$ a function $\in \bar{\Sigma}$ with content $=1$ ). Drop from $\prod_{\text {l }}$ the factors with bases $=1$. Note for $\left(i_{n+1}\right)$ : it means dropping the coinciding factors from the $(*)$ representations of $p$ and $p^{\prime}$. Note for $\left(a_{n+1}\right)$ : in case $p^{\prime}=1$ the expression $(* *)$ is still the $(*)$ representation of $p^{\prime}$. Denote $\sigma=$ 
$\left(\mu / \mu^{\prime}\right) \cdot \prod\left\{\left(\psi_{l} \uparrow \rho_{l}\right) \mid \psi_{l}=\right.$ const and $\left.\rho_{l} \in \bar{\Sigma}\right\} ;$ then $\sigma$ is a quotient of + -primes of $\bar{\Sigma}$ and

$(* * *)$

$$
p / p^{\prime}=\sigma \cdot \prod_{l}^{\prime}\left(\psi_{l} \uparrow \rho_{l}\right),
$$

where $\Pi_{l}^{\prime}$ ranges over the $\prod_{l}$ factors of $(* *)$ not incorporated into $\sigma$. In $(* * *)$ each remaining $\psi_{l}$ either is a constant (then $\rho_{l} \notin \bar{\Sigma}$ ) or has a zero or a pole in $\mathbf{C}^{m}$; see Corollary 6 of $\S 1.4$. Note for $\left(i_{n+1}\right)$ : to establish the coincidence of the $(*)$ representations of $p$ and $p^{\prime}$ it suffices to show that $\sigma=1$ and $\Pi_{l}^{\prime}$ is empty in $(* * *)$, see Proposition 1 of $\S 1.4$. Note for $\left(a_{n+1}\right)$ : in case $p^{\prime}=1$ in the expression $(* * *) \quad \sigma$ is a + -prime of $\bar{\Sigma}$.

For each $\left(\psi_{l} \uparrow \rho_{l}\right)$ shown in $(* * *)$ let $\left(T_{l}, E_{l}, b_{l}, c_{l}\right)$ be a proper $\left(\mathbf{C}^{m}, \overrightarrow{1}\right.$, $\mathscr{H})$-system with $t_{l}$ the unique sink of $\left(T_{l}, E_{l}\right)$ and $\rho_{l}=g_{t_{l}}$. (By $\left(\mathrm{a}_{n}\right)$, such $\left(\mathbf{C}^{m}, \overrightarrow{1}, \mathscr{H}\right)$-systems do exist.) Now construct $(T, E, b, c)$ as follows. Put $T=$ the disjoint union of $T_{l}$ 's and $\{t\}$, where $t$ is an extra vector. Put $E=$ the disjoint union of $E_{l}$ 's and the set of extra edges from $t_{l}$ 's to $t$. Let $\varepsilon \in E$. If $\varepsilon$ belongs to some $E_{l}$ then put $b_{\varepsilon}$ equal to $\left(b_{l}\right)_{\varepsilon}$. If $\varepsilon$ is an edge from some $t_{l}$ to $t$ then put

$$
b_{\varepsilon}= \begin{cases}1 & \text { if } \psi_{l}=\text { const }, \\ \log \psi_{l} & \text { otherwise } .\end{cases}
$$

Let $\tau \in T$. For $\tau=t$ put $c_{t}=\sigma$. If $\tau$ belongs to some $T_{l}-\left\{t_{l}\right\}$ then put $c_{\tau}$ equal to $\left(c_{l}\right)_{\tau}$. For $\tau$ being one of $\left\{t_{l}\right\}_{l}$ put

$$
c_{t_{l}}= \begin{cases}\log \psi_{l} \cdot\left(c_{l}\right)_{t_{l}} & \text { if } \psi_{l}=\text { const } \\ \left(c_{l}\right)_{t_{l}} & \text { otherwise }\end{cases}
$$

Thus $(T, E, b, c)$ is constructed. Note that $g_{t_{l}}$ retains its value from

$$
\left(T_{l}, E_{l}, b_{l}, c_{l}\right) \text {, }
$$

when $\psi_{l} \neq$ const and is multiplied by $\log \psi_{l}$ otherwise.

Obviously, $p / p^{\prime}=g_{t}$ and $(T, E, b, c)$ is a $\left(\mathbf{C}^{m}, \overrightarrow{1}, \mathscr{H}\right)$-system in case $p^{\prime}=1$. Let us show that $(T, E, b, c)$ is proper. The properness conditions need to be checked only at the edges from $t_{l}$ 's to $t$ and at the vertex $t$. By Corollary 6 of $\S 1.4$ each remaining $\psi_{l}$ is either a constant (and then $b_{\varepsilon}=1$ for the $\varepsilon$ from $t_{l}$ to $t$ ) or possesses a zero or a pole on $\mathbf{C}^{m}$ (and then $b_{\varepsilon} \notin \mathscr{P}$ for the $\varepsilon$ from $t_{l}$ to $t$ ). Besides, if $b_{\varepsilon}=1$ for an $\varepsilon$ from some $t_{l}$ to $t$, then $\rho_{l} \notin \bar{\Sigma}$ (for otherwise $\left(\psi_{l} \uparrow \rho_{l}\right)$ would be incorporated into $\sigma$ ) and hence that vertex $t_{l}$ is not a source. This shows that condition (ii) of the definition of properness holds. Consider $\left\{g_{\varepsilon} \bullet\right\}_{\varepsilon_{\bullet}=t}$. Since each $g_{t_{l}}$ in this family is (a nonzero real constant) $\cdot \rho_{l}$ and distinct +-primes $\{1\} \cup\left\{\rho_{l}\right\}_{l}$ of $\overline{\mathscr{S}}_{n}$ are linearly independent over $\mathbf{R}$ by ( $\left.\mathrm{ii}_{n}\right)$, the family $\{1\} \cup\left\{g_{t_{l}}\right\}_{l}$ is linearly independent. This shows that condition (iii) of the definition of properness holds.

For $p^{\prime}=1$ the above construction and argument already establish $\left(\mathrm{a}_{n+1}\right)$.

$\left(i_{n+1}\right)$ If $p / p^{\prime}=$ const then by Corollary 3 of $\S 4.3$ the sink $t$ of $(T, E)$ in the $\left(\mathbf{C}^{m}, \overrightarrow{1}, \mathscr{M}\right)$-system constructed above is a source of $(T, E)$. This is possible only if $T=\{t\}$ and $E=\varnothing$, i.e., when $\Pi_{l}^{\prime}$ in $(* * *)$ is empty. Consequently $p / p^{\prime}$ is a quotient of +-primes of $\bar{\Sigma}$ and those factors of their $(*)$ representations which are not of the $\left(\nabla_{0}\right)$-form coincide up to a permutation of indices. Then Proposition 1 of $\S 1.4$ implies the result. 
$\left(\mathrm{ii}_{n+1}\right)$ Let $\left\{f_{k}\right\}_{k=0}^{m} \subset \overline{\mathscr{S}_{n+1}}$ possess representations of the form $\left(\nabla_{n+1}\right)$. Suppose that $\left\{f_{k}\right\}_{k}$ are linearly dependent over $\mathbf{R}$ but every proper subfamily of $\left\{f_{k}\right\}_{k}$ is not. Let $\left\{\lambda_{k}\right\}_{k}$ be nonzero constants such that $\sum_{k=0}^{m} \lambda_{k} f_{k}=0$. Consider $h_{k}=f_{k} / f_{0}$ for $1 \leq k \leq m$; then $\lambda_{0}+\sum_{k=1}^{m} \lambda_{k} h_{k}=0$. For each $h_{k}$ let $\left(T_{k}, E_{k}, b_{k}, c_{k}\right)$ be a proper $\left(\mathbf{C}^{l}, \overrightarrow{1}, \mathscr{M}\right)$-system as described in the beginning of the proof. The disjoint union of the $\left(T_{k}, E_{k}, b_{k}, c_{k}\right)$ 's is then a proper $\left(\mathbf{C}^{l}, \overrightarrow{1}, \mathscr{M}\right)$-system with $\left\{t_{k}\right\}_{k}$ the sinks of $(T, E)$ and $h_{k}=g_{t_{k}}$ for each $k$. From $\lambda_{0}+\sum_{k=1}^{m} \lambda_{k} g_{t_{k}}=0$ by Corollary 3 of $\S 4.3$ it follows that $\sum\left\{\lambda_{k} g_{t_{k}} \mid t_{k}\right.$ is not a source of $\left.\left(T_{k}, E_{k}\right)\right\}=0$. Since every proper subfamily of $\{1\} \cup\left\{h_{k}\right\}_{k}$ is linearly independent, the latter identity implies that each $t_{k}$ is a source and each $h_{k}$ is a quotient of +-primes of $\bar{\Sigma}$. Now Proposition 1 of $\S 1.4$ implies the result.

$\left(\right.$ iii $\left._{n+1}\right)$ By $\left\{\left(\mathrm{iii}_{k}\right)\right\}_{k \leq n}$, the $\left(\nabla_{k}\right)$ representations of functions from $\overline{\mathscr{S}}_{k}$ can be regarded as the $\left(\nabla_{n+1}\right)$ representations of those functions for $k \leq n$. In view of $\left(\mathrm{i}_{n+1}\right)$ and $\left(\mathrm{ii}_{n+1}\right)$ this implies (iii $\left.n,+\right)$. Let $f=\sum_{i} f_{i}$ (perhaps with only one summand) with each $f_{i}$ of the form $\left(\nabla_{n}\right)$ and $f=\prod_{j} g_{j}$, where each $g_{j}=\sum_{k} g_{j k}$ (perhaps with only one summand) with each $g_{j k}$ of the form $\left(\nabla_{n+1}\right)$. Then $\prod_{j} g_{j}=\sum_{K} \prod_{j} g_{j, K(j)}$, where $\sum_{K}$ runs over functions $\{$ the $j-$ indices $\} \rightarrow$ the $k$-indices $\}$ such that $K(j)$ is one of the $k$ 's for which the $g_{j k}$ 's were present (for each $j$ ). By $\left\{\left(\mathrm{iii}_{k},+\right)\right\}_{k \leq n}$ and $\left(\mathrm{ii}_{n+1}\right)$, each $\prod_{j} g_{j, K(j)}$ is a +-prime of $\overline{\mathscr{S}_{n+1}}$ and hence, by $\left(\mathrm{iii}_{n+1}\right)$, is one of $\left\{f_{i}\right\}_{i}$. In the $\left(\Omega_{n+1}\right)$-form of each $\prod_{j} g_{j, K(j)}$ the exponents occurring are exactly those which occurred in the $\left(\nabla_{n+1}\right)$-forms of $\left\{g_{j k}\right\}_{j, k}$. By $\left(i_{n+1}\right)$, then all exponents occurring in the $\left(\bigcirc_{n+1}\right)$ representations of $\left\{g_{j k}\right\}_{j, k}$ belong to $\overline{\mathscr{S}_{n-1}}$, i.e., all $g_{j k} \in \overline{\mathscr{S}}_{n}$.

Corollary 2. Each function in $\overline{\mathscr{S}}=\bigcup_{n=0}^{\infty} \overline{\mathscr{S}}_{n}$ can be uniquely decomposed into a sum of functions of the form

(a monic monomial)

$$
\begin{aligned}
& \times \prod_{i}(\text { a monomial, } \neq 1)_{i} \uparrow(a+\text {-prime } \in \overline{\mathscr{S}}, \neq 1)_{i} \\
& \times \prod_{j}(\text { a function } \in \bar{\Sigma}-\{1\} \text { with content }=1)_{j} \\
& \uparrow(a+\text {-prime } \in \overline{\mathscr{S}}, \neq 1)_{j},
\end{aligned}
$$

where $\Pi_{i}$ or $\Pi_{j}$ (or both) may be empty, in each of those products all exponents occurring are distinct, and the content is taken with respect to the $(+, \cdot)$-primes of $\bar{\Sigma}$. Distinct +-primes of $\overline{\mathscr{S}}$ are linearly independent over $\mathbf{R}$. The +-primes of $\overline{\mathscr{S}}$ are: $\bigcup_{n}\left\{\right.$ the + -primes of $\left.\overline{\mathscr{S}}_{n}\right\}=\{$ the functions which can be presented in the form $(\mathcal{\nabla})\}$, the multiplicative primes of $\overline{\mathscr{S}}$ are: $\bigcup_{n}\{$ the multiplicative primes of $\left.\overline{\mathscr{S}_{n}}\right\}$.

One can ask for a description of the multiplicative primes of $\overline{\mathscr{S}}$. The multiplicative primes are always more difficult to describe than the +-primes. In the trivial case, of $\mathbf{N}$, the set of +-primes is $\{1\}$ but the set of multiplicative primes is rather complicated. In an easy case of $\mathbf{N}[x]$ the set of +-primes is $\left\{x^{\nu}\right\}_{\nu=0}^{\infty}$, but there is no easy description of the set of multiplicative primes. Instead of describing the multiplicative primes of $\overline{\mathscr{S}}$, we provide an algebraic description 
of $\overline{\mathscr{S}}$ which explains the difficulties involved. Denote by $P$ the multiplicative semigroup of elements of $\bar{\Sigma}$ having content 1. For each t-prime $f$ of $\overline{\mathscr{S}}$ let $P_{f}$ be the copy of $P$ (corresponding to functions of the form $p^{f}, p \in P$ ). Now, $\overline{\mathscr{S}}$ when considered as a semiring (i.e., with operations + , $\cdot$ only) is isomorphic to the semigroup semiring $\bar{\Sigma}\left(\bigoplus_{f} P_{f}\right)$.

Clearly, every $f \in \overline{\mathscr{L}}_{n}$ is a sum of +-primes of $\overline{\mathscr{L}_{n}}$ and every +-prime in $\overline{\mathscr{L}}_{n}$ can be presented in the form

(a monic monomial)

$$
\begin{aligned}
& \times \prod_{i}(\text { a monomial, } \neq 1)_{i} \uparrow\left(\text { a }+ \text {-prime } \in \overline{\mathscr{L}_{n-1}}, \neq 1\right)_{i} \\
& \times \prod_{j}(\text { a function } \in \bar{\Lambda}-\{1\} \text { with content }=1)_{j} \\
& \uparrow\left(\text { a }+ \text {-prime } \in \overline{\mathscr{L}_{n-1}}, \neq 1\right)_{j},
\end{aligned}
$$

where $\prod_{i}$ or $\prod_{j}$ (or both) may be empty, in each of these products all exponents occurring are distinct, and the content is taken with respect to the $(+, \cdot)$ primes of $\bar{\Lambda}$. The uniqueness of these presentations is proved by reduction to that of $\left(\nabla_{n}\right)$ with the transformation $\chi$ described in $\S 1.4$.

Proposition 3. For every $n \in \mathbf{N}$,

$\left(\mathrm{i}_{n}\right) \chi\left\{\right.$ the + -primes of $\left.\overline{\mathscr{L}_{n}}\right\} \subset\left\{\right.$ the + -primes of $\left.\overline{\mathscr{S}_{n}}\right\}$ and $\chi\{$ the $(+, \cdot)$-primes of $\left.\overline{\mathscr{L}_{n}}\right\} \subset\left\{\right.$ the $(+, \cdot)$-primes of $\left.\overline{\mathscr{S}_{n}}\right\}$.

$\left(\mathrm{ii}_{n}\right)$ Let $f, g \in \overline{\mathscr{L}}_{n}$ possess representations of the form $\left(\boldsymbol{\beta}_{n}\right)$. If $\mathrm{f} / \mathrm{g}=\mathrm{const}$ then $f=g$ and their $\left(\boldsymbol{\beta}_{n}\right)$ representations coincide up to a permutation of factors.

$\left(\right.$ iii $\left._{n}\right)$ Let $\left\{f_{k}\right\}_{k} \subset \overline{\mathscr{L}}_{n}$ possess representations of the form $\left(\boldsymbol{M}_{n}\right)$. If $f_{l} \neq f_{m}$ for $l \neq m$ then $\left\{f_{k}\right\}_{k}$ is linearly independent over $\mathbf{R}$.

$\left(\mathrm{iv}_{n}\right)$ The $\$$-primes of $\overline{\mathscr{L}_{n-1}}$ remain $\$$-prime in $\overline{\mathscr{L}_{n}}$, the $\$$-primes of $\overline{\mathscr{S}_{n}}$ remain $\$$-prime in $\overline{\mathscr{L}_{n}}$, for $\$ \in\{+, \cdot\}$.

Again, an immediate consequence of $\left(\mathrm{ii}_{n}\right)$ and $\left(\mathrm{iii}_{n}\right)$ is that the +-primes of $\overline{\mathscr{L}}_{n}$ are precisely the functions which can be presented in the form $\left(\boldsymbol{q}_{n}\right)$. The proof is almost identical to that of Proposition 3 of $\S 3.3$.

Proof. It suffices to prove the following auxiliary statement

$\left(\mathrm{a}_{n}\right) \chi\left(\right.$ every $\left(\boldsymbol{\omega}_{n}\right)$-form) can be presented by a $\left(\nabla_{n}\right)$-form without the "monic monomial" factor, and in such a way that the $\left(\nabla_{n}\right)$-forms corresponding to $\chi$ (distinct $\left(\boldsymbol{\beta}_{n}\right)$-forms) are distinct.

Proceed by induction on $n$. The cases $n=0$ and $n=1$ are contained in Corollary 4 of $\S 1.4$ and Proposition 3 of $\S 3.3$. Consider the induction step.

Let $p=\mu \cdot \prod_{i}\left(\alpha_{i} \uparrow \beta_{i}\right) \cdot \prod_{j}\left(\gamma_{j} \uparrow \delta_{j}\right)$ according to $\left(\boldsymbol{\phi}_{n+1}\right)$. Let $\mu=x^{s}$ and $\alpha_{i}=m_{i} x^{t_{i}}$, where $x=\left\{x_{k}\right\}_{k}$ is the family of the variables occurring, $m_{i} \in \mathbf{N}$, and $s,\left\{t_{i}\right\}_{i}$ are multi-indices. Then

$$
\chi(\mu)=\prod\left\{\left(a_{k}\right)^{x_{k}} \mid s_{k} \neq 0\right\},
$$

and

$$
\chi\left(\alpha_{i} \uparrow \beta_{i}\right)=\left(m_{i}\right)^{\chi\left(\beta_{i}\right)} \cdot \prod\left\{\left(b_{i, k}\right)^{x_{k} \cdot \chi\left(\beta_{i}\right)} \mid k \text { with } t_{i, k} \neq 0\right\},
$$


where $a_{k}=2^{s_{k}}$ and $b_{i, k}=2^{t_{i, k}}$. By Proposition 3 of $\S 1.4$ all $\chi\left(\gamma_{j}\right) \in \bar{\Sigma}-\{1\}$ and have content $=1$, and by the induction assumption $\left\{\chi\left(\delta_{j}\right)\right\}_{j}$ are $\neq 1$ distinct +-primes of $\overline{\mathscr{S}}_{n}$. Thus

$$
\prod_{j}\left(\chi\left(\gamma_{j}\right) \uparrow \chi\left(\delta_{j}\right)\right)
$$

forms the $\prod_{j}$-part of the $\left(Q_{n+1}\right)$ representation of $\chi(p)$. The "monic monomial" factor in the $\left(\Theta_{n+1}\right)$ representation of $\chi(p)$ will be trivial. To check that

$$
\begin{aligned}
\chi(\mu & \left.\cdot \prod_{i}\left(\alpha_{i} \uparrow \beta_{i}\right)\right) \\
& =\prod\left\{\left(a_{k}\right)^{x_{k}} \mid s_{k} \neq 0\right\} \cdot \prod\left\{\left(m_{i}\right)^{\chi\left(\beta_{i}\right)} \mid m_{i} \neq 1\right\} \cdot \prod\left\{\left(b_{i, k}\right)^{x_{k} \cdot x\left(\beta_{i}\right)} \mid t_{i, k} \neq 0\right\}
\end{aligned}
$$

does form the $\prod_{i}$-part of the $\left(Q_{n+1}\right)$ representation of $\chi(p)$ it suffices to show that the elements of

$$
\left\{x_{k}\right\}_{k} \cup\left\{\chi\left(\beta_{i}\right)\right\}_{i} \cup\left\{x_{k} \cdot \chi\left(\beta_{i}\right)\right\}_{i, k}
$$

are distinct +-primes of $\overline{\mathscr{S}_{n+1}}$. Their +-primeness is guaranteed by the induction assumption. Each $x_{k}$ consists of the "monic monomial" factor only in its $\left(O_{n+1}\right)$ representation, each $\chi\left(\beta_{i}\right)$ is $\neq 1$ and has the trivial "monic monomial" factor in its $\left(O_{n+1}\right)$ representation, each $x_{k} \cdot \chi\left(\beta_{i}\right)$ has the "monic monomial" factor $x_{k}$ and at least one of $\prod_{i}, \prod_{j}$ nontrivial in its $\left(O_{n+1}\right)$ representation. By Proposition 1 then the sets $\left\{x_{k}\right\}_{k},\left\{\chi\left(\beta_{i}\right)\right\}_{i},\left\{x_{k} \cdot \chi\left(\beta_{i}\right)\right\}_{i}$ are disjoint. The $\chi\left(\beta_{i}\right)$ are distinct because the $\beta_{i}$ are. If $x_{k} \cdot \chi\left(\beta_{i}\right)=x_{k^{\prime}} \cdot \chi\left(\beta_{i^{\prime}}\right)$ then $k=k^{\prime}$ since their "monic monomial" parts must coincide and hence $\chi\left(\beta_{i}\right)=\chi\left(\beta_{i^{\prime}}\right), i=i^{\prime}$.

Consider also $p^{\prime}=\mu^{\prime} \cdot \prod_{i^{\prime}}^{\prime}\left(\alpha_{i^{\prime}}^{\prime} \uparrow \beta_{i^{\prime}}^{\prime}\right) \cdot \prod_{j^{\prime}}^{\prime}\left(\gamma_{j^{\prime}}^{\prime} \uparrow \delta_{j^{\prime}}^{\prime}\right)$ according to $\left(\boldsymbol{\$}_{n+1}\right)$. If the $\Pi_{j^{\prime}}^{\prime}$ part of this representation of $p^{\prime}$ differs from the $\Pi_{j}$ part of $p$, say,

$$
\left(\alpha_{t}^{\prime}, \beta_{t}\right) \notin\left\{\left(\alpha_{i}, \beta_{i}\right)\right\}_{i},
$$

then the similar difference

$$
\left(\chi\left(\alpha_{t}^{\prime}\right), \chi\left(\beta_{t}^{\prime}\right)\right) \notin\left\{\left(\chi\left(\alpha_{i}\right), \chi\left(\beta_{i}\right)\right)\right\}_{i}
$$

will occur between the $\left(Q_{n+1}\right)$ representations of $\chi(p)$ and $\chi\left(p^{\prime}\right)$ constructed above. Suppose that the $\prod_{i}$-parts of the $\left(Q_{n+1}\right)$ representations of $\chi(p)$ and $\chi\left(p^{\prime}\right)$ coincide. Then

$$
\left\{x_{k}\right\}_{k} \cup\left\{\chi\left(\beta_{i}\right)\right\}_{i} \cup\left\{x_{k} \cdot \chi\left(\beta_{i}\right)\right\}_{i, k}
$$

and

$$
\left\{x_{k^{\prime}}\right\}_{k^{\prime}} \cup\left\{\chi\left(\beta_{i^{\prime}}^{\prime}\right)\right\}_{i^{\prime}} \cup\left\{x_{k^{\prime}} \cdot \chi\left(\beta_{i^{\prime}}^{\prime}\right)\right\}_{i^{\prime}, k^{\prime}}
$$

are the same set of +-primes of $\overline{\mathscr{S}_{n}}$. The comparison of the "monic monomial" parts of their $\left(Q_{n}\right)$ representations (as above) shows that $\left\{x_{k}\right\}_{k}=\left\{x_{k^{\prime}}\right\}_{k^{\prime}}$, $\left\{\chi\left(\beta_{i}\right)\right\}_{i}=\left\{\chi\left(\beta_{i^{\prime}}^{\prime}\right)\right\}_{i^{\prime}}$, and $\left\{x_{k} \cdot \chi\left(\beta_{i}\right)\right\}_{i, k}=\left\{x_{k^{\prime}} \cdot \chi\left(\beta_{i^{\prime}}^{\prime}\right)\right\}_{i^{\prime}, k^{\prime}}$. Consequently there is a bijection $\sigma$ from the $i$-indices into the $i^{\prime}$-indices such that $\beta_{\sigma(i)}^{\prime}=$ $\beta_{i}$. The comparison of the bases in the factors with exponents belonging to $\left\{\chi\left(\beta_{i}\right)\right\}_{i}$ of the $\prod_{i}$ parts of the $\left(O_{n+1}\right)$ representations of $\chi(p)$ and $\chi\left(p^{\prime}\right)$ 
shows then that $m_{\sigma(i)}^{\prime}=m_{i}$. Since $x_{k} \cdot \chi\left(\beta_{i}\right)=x_{k^{\prime}} \cdot \chi\left(\beta_{i^{\prime}}^{\prime}\right)$ implies $k=k^{\prime}$ and $\beta_{i}=\beta_{i^{\prime}}^{\prime}$ (still by the comparison of the "monic monomial" parts of their $\left(O_{n}\right)$ representations), the multi-indices corresponding to the monomials mentioned in the $\left(\phi_{n+1}\right)$ representations of $p$ and $p^{\prime}$ also coincide: $s=s^{\prime}, t_{\sigma(i)}^{\prime}=t_{i}$. Thus the $\prod_{j}$-parts of $\chi(p), \chi\left(p^{\prime}\right)$ tell whether $p=p^{\prime}$ or not. The identity $p=p^{\prime}$ holds iff $\chi(p)=\chi\left(p^{\prime}\right)$ and there exists a bijection $\sigma:\{j\}_{j} \rightarrow\left\{j^{\prime}\right\}_{j^{\prime}}$ such that $\chi\left(\gamma_{j}\right)=\chi\left(\gamma_{\sigma(j)}^{\prime}\right), \chi\left(\delta_{j}\right)=\chi\left(\delta_{\sigma(j)}^{\prime}\right)$. Then $\gamma_{j}=\gamma_{\sigma(j)}$ and $\delta_{j}=\delta_{\sigma(j)}$.

Corollary 4. Each function in $\overline{\mathscr{L}}=\bigcup_{n=0}^{\infty} \overline{\mathscr{L}_{n}}$ can be uniquely decomposed into a sum of functions of the form

(a monic monomial)

(\$)

$$
\begin{aligned}
& \times \prod_{i}(\text { a monomial, } \neq 1)_{i} \uparrow(a+\text {-prime } \in \overline{\mathscr{L}}, \neq 1)_{i} \\
& \times \prod_{j}(\text { a function } \in \bar{\Lambda}-\{1\} \text { with content }=1)_{j} \\
& \uparrow(a+\text {-prime } \in \overline{\mathscr{L}}, \neq 1)_{j},
\end{aligned}
$$

where $\Pi_{i}$ or $\Pi_{j}$ (or both) may be empty, in each of these products all exponents occurring are distinct, and the content is taken with respect to the $(+, \cdot)$-primes of $\bar{\Lambda}$. Distinct +-primes of $\overline{\mathscr{L}}$ are linearly independent over $\mathbf{R}$. The +-primes of $\overline{\mathscr{L}}$ are: $\bigcup_{n}\left\{\right.$ the +-primes of $\left.\overline{\mathscr{L}_{n}}\right\}=\{$ the functions which can be presented in the form (\$) \}, the multiplicative primes of $\overline{\mathscr{L}}$ are: $\bigcup_{n}\{$ the multiplicative primes of $\overline{\mathscr{L}_{n}}$. The +-primes (the multiplicative primes) of $\overline{\mathscr{S}}$ are +-prime (resp. multiplicatively prime) in $\overline{\mathscr{L}}$.

Proposition 5. Tarski's conjecture holds on $\mathscr{L}$, i.e., if $t, u \in \mathscr{L}$ and $\bar{t}=\bar{u}$ then $\mathscr{T} \vdash t=u$.

Proof. It is easy to see that for all $n$ each $t \in \mathscr{L}_{n}$ is $\mathscr{T}$-equivalent to a single term or to a sum of terms in $\mathscr{L}_{n}$ having the form $\left(\boldsymbol{q}_{n}\right)$ sans overlining,

$$
a \cdot \prod_{j}\left(b_{i} \uparrow p_{i}\right) \cdot \prod_{j}\left(c_{j} \uparrow q_{j}\right),
$$

where

$a$ is a monic monomial, i.e., either 1 or a product of variables, each $b_{i}$ is a monomial, i.e., either a constant or (a constant) $\times(\mathrm{a}$ product of variables), and $\overline{b_{i}} \neq 1$,

$p_{i} \in \mathscr{L}_{\max (0, n-1)}, \bar{p}_{i}$ is a +-prime of $\overline{\mathscr{L}}_{\max (0, n-1)}, \bar{p}_{i} \neq 1$ for each $i$, and $\overline{p_{i}} \neq \overline{p_{i^{\prime}}}$ for $i \neq i^{\prime}$,

(" $\prod_{i} \ldots$ " may be empty),

and in case $n>0$ (otherwise " $\prod_{j} \ldots$ " is absent),

$c_{j} \in \Lambda, \bar{c}_{j}$ has content 1 in $\bar{\Lambda}$, and $\overline{c_{j}} \neq 1$ for each $j$,

$q_{j} \in \mathscr{L}_{n-1}, \overline{q_{j}}$ is a +-prime of $\overline{\mathscr{L}_{n-1}}, \overline{q_{j}} \neq 1$ for each $j$, and $\overline{q_{j}} \neq \overline{q_{j^{\prime}}}$ for $j \neq j^{\prime}$,

(" $\Pi_{j} \ldots$ " may be empty).

To prove that for each $n, t, u \in \mathscr{L}_{n}$ the functional identity $\bar{t}=\bar{u}$ implies $t=g u$ proceed by induction on $n$. The induction base $n=0$ is proved in 
§1.4. Consider the induction step $n-1 \mapsto n$. Let $t=\mathscr{g} t^{\prime}$ and $u=g u^{\prime}$, where $t^{\prime}$ and $u^{\prime}$ are sums of $(\boldsymbol{\$})$ terms. By Corollary 4 the summands of $t^{\prime}$ and $u^{\prime}$ are in a bijective correspondence such that the functions determined by the corresponding summands coincide. Thus it suffices to consider the case of $t^{\prime}, u^{\prime}$ consisting of only one ( $\left.\boldsymbol{\phi}\right)$ term each. Then, by Corollary 4 , the factors in $t^{\prime}$ and $u^{\prime}$ are in a bijective correspondence such that in each pair of the corresponding factors of $t^{\prime}$ and $u^{\prime}$ the bases determine the same function and the exponents determine the same function. Since those bases are in $\Lambda$ and those exponents are in $\mathscr{L}_{n-1}$, the induction assumption implies that the corresponding bases are $\mathscr{T}$-equivalent and the corresponding exponents are $\mathscr{T}$-equivalent.

\section{ACKNOWLEDGMENTS}

This paper is a slightly revised version of my $\mathrm{Ph} . \mathrm{D}$. thesis at the University of Illinois at Urbana-Champaign.

A partial list of people to whom I wish to express my gratitude on the occasion of the completion of my Ph.D. project is J. d'Angelo, R. Graggs, L. van den Dries, Yu. Sh. Gurevich, H. Halberstam, C. W. Henson, B. I. Kunin, I.-j. Lin, J. Miles, G. E. Mints, E. Movshovich, L. A. Rubel, S. Schneider, D. H. Seigel.

Of these, I wish to mention specially G. E. Mints who attracted my attention to Tarski's high school algebra problem and L. A. Rubel who was a great advisor throughout my stay in Urbana-Champaign.

\section{REFERENCES}

1. P. S. Aleksandrov, Introduction to homological dimension theory, Izdat. "Nauka", Moscow, 1975. (Russian) MR 58\# 24234.

2. P. S. Aleksandrov and B. A. Pasynkov, Introduction to dimension theory, Izdat. "Nauka", Moscow, 1973. (Russian) MR 51 \#1776.

3. V. I. Arnold, Supplementary chapters of the theory of ordinary differential equations, Moscow, 1978. (Russian); English transl. in Geometrical methods in the theory of ordinary differential equations, Springer-Verlag, 1983.

4. G. Birkhoff, Lattice theory, 3rd ed., Amer. Math. Soc., Providence, R. I., 1967.

5. M. Boshernitzan, An extension of Hardy's class $L$ of "orders of infinity", J. Analyse Math. 39 (1981), 235-255.

6. __ New "orders of infinity", J. Anal. Math. 41 (1982), 130-167.

7. B. I. Dahn, Fine structure of the integral exponential functions below $2^{2^{x}}$, Trans. Amer. Math. Soc. 297 (1986), 707-716.

8. L. van den Dries, Exponential rings, exponential polynomials and exponential functions, Pacific J. Math. 113 (1984), 51-66.

9. __, A generalization of Tarski-Seidenberg theorem, and some nondefinability results, Bull. Amer. Math. Soc. (N. S.) 15 (1986), 189-193.

10. __ Restricted theory of elementary functions, J. Symbolic Logic (to appear).

11. L. van den Dries and H. Levitz, On Skolem's exponential functions below $2^{2^{x}}$, Trans. Amer. Math. Soc. 286 (1984), 339-349.

12. A. Ehrenfeucht, Polynomial functions with exponentiation are well ordered, Algebra Universalis 3 (1973), 261-349.

13. R. Godement, Topologie algébrique et théorie des faisceaux, Hermann, Paris, 1958.

14. R. C. Gunning and H. Rossi, Analytic functions of several complex variables, Prentice-Hall, Englewood Cliffs, N. J., 1965. 
15. R. Gurevič, The equational theory of positive numbers with exponentiation is not finitely axiomatizable, Ann. Pure Appl. Logic (to appear).

16. D. Handelman, Deciding eventual positivity of polynomials, Ergodic Theory Dynamical Systems 6 (1986), 57-79.

17. W. K. Hayman, Meromorphic functions, Clarendon Press, Oxford, 1964.

18. L. Henkin, The logic of equality, Amer. Math. Monthly 84 (1977), 597-612.

19. C. W. Henson and L. A. Rubel, Some applications of Nevanlinna theory to mathematical logic: identities of exponential functions, Trans. Amer. Math. Soc. 282 (1984), 1-32.

20. E. Hewitt and K. A. Ross, Abstract harmonic analysis, vols. 1, 2, Springer-Verlag, 1963, 1970.

21. G. Hiromi and M. Ozawa, On the existence of analytic mappings between two ultrahyperelliptic surfaces, Kōdai Math. Sem. Rep. 17 (1965), 281-306.

22. L. Hörmander, An introduction to complex analysis in several variables, Van Nostrand, Princeton, N. J., 1966.

23. W. Hurewicz and H. Wallman, Dimension theory, 2nd ed. , Princeton Univ. Press, Princeton, N. J., 1948.

24. I. Kaplansky, An introduction to differential algebra, Hermann, Paris, 1957.

25. K. Kuratowski and A. Mostowski, Set theory, North-Holland, Amsterdam, 1967.

26. S. Lang, Algebra, Addison-Wesley, 1965.

27. S. Lefschetz, On locally connected and related sets, Ann. of Math. (2) 35 (1934), 118-129.

28. B. M. Levitan and V. V. Zhikov, Almost periodic functions and differential equations, Moscow University, 1978; English transl., Cambridge Univ. Press, 1982.

29. H. Levitz, An ordinal bound for the set of polynomial functions with exponentiation, Algebra Universalis 8 (1978), 223-243.

30. A. Macintyre, Notes on exponentiation, Lecture notes, University of Illinois at UrbanaChampaign (unpublished).

31. C. B. Morrey, The analytic embedding of abstract real-analytic manifolds, Ann. of Math. (2) 68 (1958), 159-201.

32. J. T. Schwartz and M. Sharir, Motion planning and related geometric algorithms, Proc. Internat. Congr. Math. (Berkeley, 1986), pp. 1594-1611.

33. B. V. Shabat, Introduction to complex analysis. II, Moscow, 1985. (Russian)

34. P. H. Slessenger, Ph.D. Thesis, Leeds University, Leeds, 1984.

35. E. Spanier, Algebraic topology, McGraw-Hill, New York, 1966.

36. A. J. Wilkie, On exponentiation - a solution to Tarski's high school algebra problem, Oxford, 1980 (manuscript).

Department of Mathematics, University of Illinois at Urbana-Champaign, Urbana, ILLINOIS 61801

Current address: Department of Mathematics, University of Wisconsin, Madison, Wisconsin 53706 (Author deceased) 